Supporting Information

\title{
Delocalization Effects and Tunable Emission in a Class of Charged Cyclazines with Nitrogen on the Periphery
}

Jais Kurian, ${ }^{[a]}$ Kanneth S Shurooque, ${ }^{[b]}$ Venkatachalam Ramkumar, ${ }^{[a]}$ Lakshmi Chakkumkumarath ${ }^{[b]}{ }^{*}$ and Muraleedharan Kannoth M. ${ }^{[a]}$ *

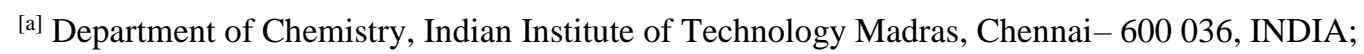

E mail: mkm@iitm.ac.in

${ }^{[b]}$ Department of Chemistry, National Institute of Technology Calicut, Kerala -673601, INDIA

\section{Contents:}

$\begin{array}{ll}\text { General information } & 02\end{array}$

Experimental procedures, ${ }^{1} \mathrm{H}$ and ${ }^{13} \mathrm{C}$ NMR data $\quad 03-07$

X-ray crystallographic data of $\mathbf{3 d}$ and $\mathbf{4 d} \quad 08-13$

$\begin{array}{lr}\text { Photophysical characterization } & \text { 14-16 }\end{array}$

$\begin{array}{ll}\text { TD-DFT studies } & 17-18\end{array}$

${ }^{1} \mathrm{H}$ and ${ }^{13} \mathrm{C}$ NMR spectra $\quad 19-50$

HRMS spectra of compounds 4a-g $\quad$ 51-54

References $\quad 54$ 


\section{General information:}

All starting materials and reagents were purchased from Spectrochem or Sigma Aldrich, and used without further purification. The solvents tetrahydrofuran (THF) and toluene (PhMe) were dried over sodium/benzophenone, and acetonitrile $\left(\mathrm{CH}_{3} \mathrm{CN}\right)$ was dried over $\mathrm{CaH}_{2}$. Methanol was dried by refluxing over magnesium/iodine. The reagent-grade chloroform was directly used for the synthesis. Column chromatographic purification was carried out on 100-200 mesh silica gel using EtOAc-hexane solvent system in a gradient mode. Thin layer chromatography was done using $0.25 \mathrm{~mm}$ thick silica gel plates from Merck and was analysed using either $254 \mathrm{~nm}$ UV light or ninhydrin staining. Bruker Avance $500 \mathrm{MHz}$ NMR spectrometer was used to record ${ }^{1} \mathrm{H}$ and ${ }^{13} \mathrm{C}$ NMR spectra and the chemical shifts are reported in parts per million ( $\mathrm{ppm}$ ) relative to tetramethylsilane as the standard. Infrared spectra were recorded using Nicolet 6700 FT-IR spectrometer. High-resolution mass spectra (HRMS) were recorded on a Waters Q-TOF micro ${ }^{\mathrm{TM}}$ spectrometer with lock spray source.

Single crystal X-ray crystallography: The intensity data collection during X-ray crystallographic analysis was carried out on a Bruker AXS (kappa apex II) diffractometer equipped with graphite monochromated Mo $\left(\mathrm{K}_{\alpha}\right)$ radiation. The data were collected for $\theta$ up to $25^{\circ}$ for Mo $\left(\mathrm{K}_{\alpha}\right)$ radiation. $\omega$ and $\phi$ scans were employed to collect the data. The frame width for $\omega$ was set to $0.5 \mathrm{deg}$ for data collection. The frames were integrated and data were reduced for Lorentz and polarization correction using Bruker SAINT. The multi-scan absorption correction was applied to the data. All structures were solved using Bruker SHELXS-97 and refined using SHELXL-2014/7. The molecular and packing diagrams were produced using ORTEP -3 and Mercury 1.4.2. The non-hydrogen atoms were refined with anisotropic displacement parameter. All hydrogen atoms could be located in the difference Fourier map. However, the atoms bonded carbons were fixed at chemically meaningful positions and were allowed to ride with parent atom during the refinement. 


\section{Experimental procedure}

\section{Synthesis of polysubstituted indolizinones (1a-g)}

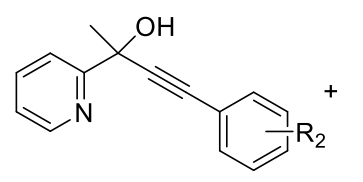<smiles>Ic1cc[R]cc1</smiles>
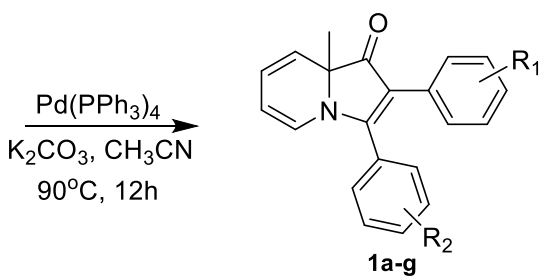

1a; $\mathrm{R}_{1}=\mathrm{H}, \mathrm{R}_{2}=\mathrm{H}$

1b; $\mathrm{R}_{1}=\mathrm{H}, \mathrm{R}_{2}=\mathrm{OMe}$

1c; $\mathrm{R}_{1}=\mathrm{OMe}, \mathrm{R}_{2}=\mathrm{H}$

1d; $\mathrm{R}_{1}=\mathrm{OMe}, \mathrm{R}_{2}=\mathrm{OMe}$

1e; $\mathrm{R}_{1}=\mathrm{NO}_{2}, \mathrm{R}_{2}=\mathrm{H}$

1f; $\mathrm{R}_{1}=\mathrm{NO}_{2}, \mathrm{R}_{2}=\mathrm{OMe}$

1g; $\mathrm{R}_{1}=\mathrm{NO}_{2}, \mathrm{R}_{2}=\mathrm{NO}_{2}$

Polysubstituted indolizinones (1a-g) were synthesized using the method reported by Cho et, al. ${ }^{1}$

Scheme for the synthesis of 3a-g

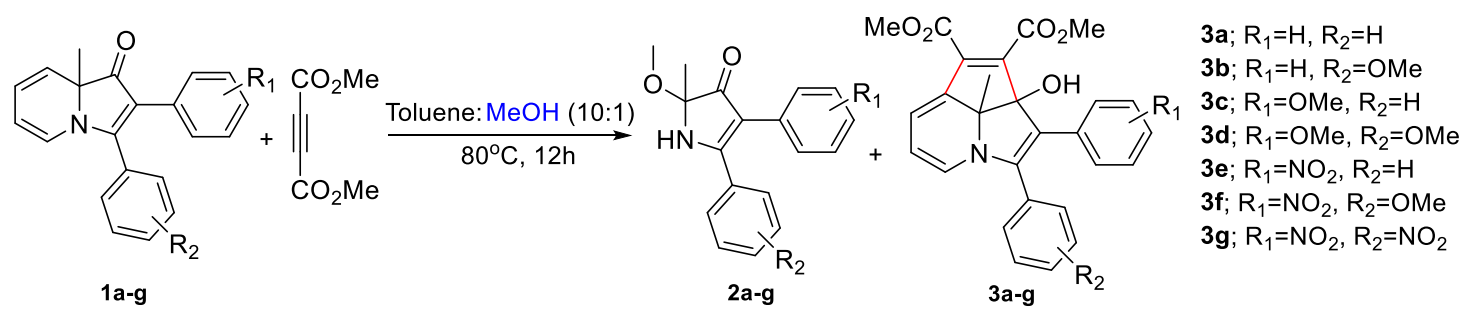

Typical procedure: A solution of Indolizinone 1a $(0.5 \mathrm{~g}, 1.6 \mathrm{mmol})$ and dimethyl acetylene dicarboxylate $(0.3 \mathrm{~mL}, 2.5 \mathrm{mmol})$ in toluene-methanol mixture $(16.5 \mathrm{~mL}, 10: 1)$ taken in a sealed tube under inert condition was heated at $80^{\circ} \mathrm{C}$ (oil bath) for $12 \mathrm{~h}$. The reaction mixture was then concentrated under reduced pressure and the crude product was purified on silica gel column using ethyl acetatehexane system to get $\mathbf{3 a}$ in $57 \%$ yield.

Dimethyl

8a-hydroxy-3'-methyl-1,2-diphenyl-3',8a-dihydrocyclopenta[hi]indolizine-7,8 dicarboxylate (3a)

$57 \%$ yield $\left(420 \mathrm{mg}\right.$ ). Orange solid, m.p. $150-152{ }^{\circ} \mathrm{C} . R f=0.53$ (40\% EtOAc/Hexane). ${ }^{1} \mathrm{H}$ NMR (500 $\left.\mathrm{MHz}, \mathrm{CDCl}_{3}\right): \delta 7.22-7.24(\mathrm{~m}, 3 \mathrm{H}, \mathrm{ArH}), 7.16-7.19(\mathrm{~m}, 5 \mathrm{H}, \mathrm{ArH}) 7.07-7.09(\mathrm{~m}, 2 \mathrm{H}, \mathrm{ArH}), 6.84$ $(\mathrm{d}, J=6.69 \mathrm{~Hz}, 1 \mathrm{H}, \mathrm{CH}), 6.68(\mathrm{~d}, J=5.81 \mathrm{~Hz}, 1 \mathrm{H}, \mathrm{CH}), 5.91$ (appt, $J=6.37 \mathrm{~Hz}, 1 \mathrm{H}, \mathrm{CH}), 4.28(\mathrm{~s}, 1 \mathrm{H}$, $\mathrm{OH}), 3.93$ (s, $\left.3 \mathrm{H}, \mathrm{OCH}_{3}\right), 3.41$ (s, 3H, OCH 3 ), 0.80 (s, 3H, CH 3 ppm. ${ }^{13} \mathrm{C} \mathrm{NMR}\left(125 \mathrm{MHz}, \mathrm{CDCl}_{3}\right): \delta$ 165.4, 165.3, 144.9, 143.8, 133.6, 132.1 (2C), 129.9, 129.4 (2C), 129.0, 128.9, 128.4 (2C), 127.6 (2C), 127.0, 126.2, 124.4, 119.8, 109.7, 109.6, 91.9, 68.7, 52.7, 51.8, 13.3 ppm. IR (KBr): 3473, 2955, 2923, $1723,1568,1443,1292,1232,737,704 \mathrm{~cm}^{-1}$. HRMS (ESI) m/z: [M+H] ${ }^{+}$calcd for $\mathrm{C}_{27} \mathrm{H}_{24} \mathrm{NO}_{5} 442.1654$; found 442.1652 .

\section{Dimethyl 8a-hydroxy-2-(4-methoxyphenyl)-3'-methyl-1-phenyl-3',8a-dihydrocyclopenta[hi] indolizine-7,8-dicarboxylate (3b)}

Reaction of $\mathbf{1 b}(0.5 \mathrm{~g}, 1.5 \mathrm{mmol})$ with DMAD $(0.28 \mathrm{~mL}, 2.3 \mathrm{mmol})$ as per the protocol described above for the synthesis of $\mathbf{3 a}$ afforded the product $\mathbf{3 b}$ in $66 \%$ yield $(472 \mathrm{mg})$. Orange solid, m.p. $92-94{ }^{\circ} \mathrm{C}$. $R f=0.50\left(40 \%\right.$ EtOAc/Hexane). ${ }^{1} \mathrm{H}$ NMR $\left(500 \mathrm{MHz}, \mathrm{CDCl}_{3}\right): \delta 7.17-7.18(\mathrm{~m}, 3 \mathrm{H}, \mathrm{ArH}), 7.07-7.11$ $(\mathrm{m}, 4 \mathrm{H}, \operatorname{ArH}), 6.87(\mathrm{~d}, J=6.53 \mathrm{~Hz}, 1 \mathrm{H}, \operatorname{ArH}), 6.75(\mathrm{~d}, J=8.88 \mathrm{~Hz}, 2 \mathrm{H}, \operatorname{ArH}), 6.67(\mathrm{~d}, J=5.80 \mathrm{~Hz}$, $1 \mathrm{H}, \mathrm{ArH}), 5.91$ (appt, $J=6.25 \mathrm{~Hz}, 1 \mathrm{H}, \mathrm{ArH}), 4.25(\mathrm{~s}, 1 \mathrm{H}, \mathrm{OH}), 3.93\left(\mathrm{~s}, 3 \mathrm{H}, \mathrm{OCH}_{3}\right), 3.74\left(\mathrm{~s}, 3 \mathrm{H}, \mathrm{OCH}_{3}\right)$, 3.41 (s, 3H, $\mathrm{OCH}_{3}$ ), 0.78 (s, 3H, $\mathrm{CH}_{3}$ ) ppm. ${ }^{13} \mathrm{C} \mathrm{NMR}\left(125 \mathrm{MHz}, \mathrm{CDCl}_{3}\right): \delta 165.5,165.3,159.8,144.7$, 143.7, 133.9, 132.2 (2C), 130.7 (2C), 129.0, 127.6 (2C), 126.9, 126.1, 124.3, 122.0, 119.8, 113.9 (2C), 
109.5, 108.9, 91.9, 68.6, 55.3, 52.7, 51.8, 13.3 ppm. IR (KBr): 3482, 2948, 2846, 1731, 1605, 1507, 1440, 1359, 1286, 1246, 1181, 1027, 840, 704, 643, 544, $\mathrm{cm}^{-1}$. HRMS (ESI) m/z: $[\mathrm{M}+\mathrm{H}]^{+}$calcd for $\mathrm{C}_{28} \mathrm{H}_{26} \mathrm{NO}_{6} 472.1760$; found 472.1755 .

\section{Dimethyl 8a-hydroxy-1-(4-methoxyphenyl)-3'-methyl-2-phenyl-3',8a-dihydrocyclopenta[hi] indolizine-7,8-dicarboxylate $(3 \mathrm{c})$}

Reaction of $1 \mathbf{c}(0.5 \mathrm{~g}, 1.5 \mathrm{mmol})$ with DMAD $(0.28 \mathrm{~mL}, 2.3 \mathrm{mmol})$ as per the protocol described above for the synthesis of 3a afforded the product $\mathbf{3 c}$ in $58 \%$ yield (414 mg). Yellow solid, m.p. $152-154$ ${ }^{\circ} \mathrm{C} . R f=0.45(40 \% \mathrm{EtOAc} / \mathrm{Hexane}) .{ }^{1} \mathrm{H} \mathrm{NMR}\left(500 \mathrm{MHz}, \mathrm{CDCl}_{3}\right): \delta 7.24-7.25(\mathrm{~m}, 3 \mathrm{H}, \mathrm{ArH}), 7.17-$ $7.18(\mathrm{~m}, 2 \mathrm{H}, \operatorname{ArH}), 7.00$ (d, $J=8.21 \mathrm{~Hz}, 2 \mathrm{H}, \operatorname{ArH}), 6.83$ (d, $J=6.45 \mathrm{~Hz}, 1 \mathrm{H}, \mathrm{CH}), 6.70$ (d, $J=8.31$ $\mathrm{Hz}, 2 \mathrm{H}, \mathrm{ArH}), 6.67$ (d, J = 5.74 Hz, 1H, CH), 5.90 (appt, $J=6.21 \mathrm{~Hz}, 1 \mathrm{H}, \mathrm{CH}), 4.29$ (s, 1H, OH), 3.93

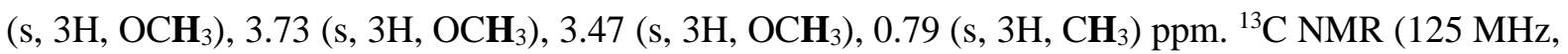
$\left.\mathrm{CDCl}_{3}\right): \delta 165.43,165.38,158.7,144.9,143.6,133.2(2 \mathrm{C}), 130.0,129.4(2 \mathrm{C}), 129.0,128.8,128.4(2 \mathrm{C})$, 126.1, 125.5, 124.4, 119.8, 113.1 (2C), 109.5, 109.2, 91.9, 68.6, 55.2, 52.7, 51.9, 13.2 ppm. IR (KBr): 3482, 2924, 1729, 1605, 1507, 1567, 1459, 1366, 1286, 1239, 1190, 1030, $737 \mathrm{~cm}^{-1}$. HRMS (ESI) m/z: $[\mathrm{M}+\mathrm{H}]^{+}$calcd for $\mathrm{C}_{28} \mathrm{H}_{26} \mathrm{NO}_{6} 472.1760$; found 472.1764 .

\section{Dimethyl 8a-hydroxy-1,2-bis(4-methoxyphenyl)-3'-methyl-3',8a-dihydrocyclopenta[hi]indolizine- 7,8-dicarboxylate (3d)}

Reaction of 1d $(0.5 \mathrm{~g}, 1.4 \mathrm{mmol})$ with DMAD $(0.26 \mathrm{~mL}, 2.0 \mathrm{mmol})$ as per the protocol described above for the synthesis of 3a afforded the product 3d in $69 \%$ yield $(480 \mathrm{mg})$. Brown solid, m.p. $132-134{ }^{\circ} \mathrm{C}$. $R f=0.43$ (40\% EtOAc/Hexane). ${ }^{1} \mathrm{H}$ NMR $\left(500 \mathrm{MHz}, \mathrm{CDCl}_{3}\right): \delta 7.10(\mathrm{~d}, J=8.91 \mathrm{~Hz}, 2 \mathrm{H}, \mathrm{ArH})$, ), 6.99 $(\mathrm{d}, J=8.63 \mathrm{~Hz}, 2 \mathrm{H}, \operatorname{ArH}),), 6.87(\mathrm{~d}, J=6.40 \mathrm{~Hz}, 1 \mathrm{H}, \mathrm{CH}), 6.76(\mathrm{~d}, J=8.91 \mathrm{~Hz}, 2 \mathrm{H}, \operatorname{ArH}), 6.71(\mathrm{~d}, J$ $=8.73 \mathrm{~Hz}, 2 \mathrm{H}, \mathrm{ArH}), 6.66(\mathrm{~d}, J=5.85 \mathrm{~Hz}, 1 \mathrm{H}, \mathrm{CH}), 5.89$ (appt, $J=5.97 \mathrm{~Hz}, 1 \mathrm{H}, \mathrm{CH}), 4.25(\mathrm{~s}, 1 \mathrm{H}$, $\mathrm{OH}), 3.92\left(\mathrm{~s}, 3 \mathrm{H}, \mathrm{OCH}_{3}\right), 3.74\left(\mathrm{~s}, 3 \mathrm{H}, \mathrm{OCH}_{3}\right), 3.73\left(\mathrm{~s}, 3 \mathrm{H}, \mathrm{OCH}_{3}\right), 3.46\left(\mathrm{~s}, 3 \mathrm{H}, \mathrm{OCH}_{3}\right), 0.77$ (s, 3H, $\left.\mathrm{CH}_{3}\right)$ ppm. ${ }^{13} \mathrm{C}$ NMR $\left(125 \mathrm{MHz}, \mathrm{CDCl}_{3}\right): \delta 165.5,165.4,159.8,158.6,144.7,143.5,133.2(2 \mathrm{C}), 130.8$ (2C), 129.0, 126.0, 125.8, 124.3, 122.1, 119.8, 113.9 (2C), 113.2 (2C), 109.4, 108.5, 91.8, 68.6, 55.3, 55.2, 52.7, 51.9, 13.2 ppm. IR (KBr): 3473, 2947, 2845, 1731, 1605, 1568, 1510, 1442, 1359, 1285 , 1245, 1177, 1103, 1062, 1030, 837, 732, $460 \mathrm{~cm}^{-1}$. HRMS (ESI) m/z: $[\mathrm{M}+\mathrm{H}]^{+}$calcd for $\mathrm{C}_{29} \mathrm{H}_{28} \mathrm{NO}_{7}$ 502.1866; found 502.1860.

\section{Dimethyl 8a-hydroxy-3'-methyl-1-(4-nitrophenyl)-2-phenyl-3',8a-dihydrocyclopenta[hi] indolizine- 7,8-dicarboxylate (3e)}

Reaction of $1 \mathrm{e}(0.5 \mathrm{~g}, 1.45 \mathrm{mmol})$ with $\mathrm{DMAD}(0.27 \mathrm{~mL}, 2.2 \mathrm{mmol})$ as per the protocol described above for the synthesis of 3a afforded the product 3e in 56\% yield (363 mg). Orange solid, m.p. $124-126$ ${ }^{\circ} \mathrm{C} . R f=0.43$ (40\% EtOAc/Hexane). ${ }^{1} \mathrm{H}$ NMR $\left(500 \mathrm{MHz}, \mathrm{CDCl}_{3}\right): \delta 7.99(\mathrm{~d}, J=8.45 \mathrm{~Hz}, 2 \mathrm{H}, \mathrm{ArH})$, $7.25-7.32(\mathrm{~m}, 5 \mathrm{H}, \operatorname{ArH}), 7.12(\mathrm{~d}, J=6.63 \mathrm{~Hz}, 2 \mathrm{H}, \operatorname{ArH}), 6.79(\mathrm{~d}, J=6.63 \mathrm{~Hz}, 1 \mathrm{H}, \mathrm{CH}), 6.72(\mathrm{~d}, J=$ $5.53 \mathrm{~Hz}, 1 \mathrm{H}, \mathrm{CH}), 6.00$ (appt, $J=6.48 \mathrm{~Hz}, 1 \mathrm{H}, \mathrm{CH}), 4.34$ (s, 1H, OH), 3.93 (s, 3H, OCH 3 ), 3.54 (s, $\left.3 \mathrm{H}, \mathrm{OCH}_{3}\right), 0.80\left(\mathrm{~s}, 3 \mathrm{H}, \mathrm{CH}_{3}\right) \mathrm{ppm} .{ }^{13} \mathrm{C} \mathrm{NMR}\left(125 \mathrm{MHz}, \mathrm{CDCl}_{3}\right): \delta 165.7,164.9,146.63,146.55$, 143.2, 142.0, 132.5 (2C), 129.5, 129.4 (2C), 129.3, 128.8 (2C), 128.7, 127.2, 125.6, 122.7 (2C), 120.0, 110.8, 107.3, 92.3, 69.0, 52.8, 52.2, 13.2 ppm. IR (KBr): 3468, 2940, 2851, 1725, 1591, 1511, 1439, $1341,1236,1107,1060,1004,852,702,530 \mathrm{~cm}^{-1}$. HRMS (ESI) m/z: $[\mathrm{M}+\mathrm{H}]^{+}$calcd for $\mathrm{C}_{27} \mathrm{H}_{23} \mathrm{~N}_{2} \mathrm{O}_{7}$ 487.1505; found 487.1476 . 

indolizine-7,8-dicarboxylate (3f)

Reaction of $\mathbf{1 f}(0.5 \mathrm{~g}, 1.3 \mathrm{mmol})$ with DMAD $(0.25 \mathrm{~mL}, 2.0 \mathrm{mmol})$ as per the protocol described above for the synthesis of $\mathbf{3 a}$ afforded the product $\mathbf{3 f}$ in $55 \%$ yield $(412 \mathrm{mg})$. Orange solid, m.p. $58-60{ }^{\circ} \mathrm{C}$. $R f=0.43$ (40\% EtOAc/Hexane). ${ }^{1} \mathrm{H}$ NMR $\left(500 \mathrm{MHz}, \mathrm{CDCl}_{3}\right): \delta 7.99(\mathrm{~d}, J=8.84 \mathrm{~Hz}, 2 \mathrm{H}, \mathrm{ArH}), 7.26$ $(\mathrm{d}, J=8.79 \mathrm{~Hz}, 2 \mathrm{H}, \operatorname{ArH}), 7.04(\mathrm{~d}, J=8.79 \mathrm{~Hz}, 2 \mathrm{H}, \operatorname{ArH}), 6.82(\mathrm{~d}, J=6.64 \mathrm{~Hz}, 1 \mathrm{H}, \mathrm{CH}), 6.80$ (d, $J=$ $8.90 \mathrm{~Hz}, 2 \mathrm{H}, \mathrm{ArH}), 6.70$ (d, $J=5.75 \mathrm{~Hz}, 1 \mathrm{H}, \mathrm{CH}), 5.99$ (appt, $J=6.45 \mathrm{~Hz}, 1 \mathrm{H}, \mathrm{CH}), 4.31$ (s, $1 \mathrm{H}, \mathrm{OH}$ ), 3.92 (s, 3H, OCH 3 ), 3.76 (s, 3H, OCH 3 ), 3.53 (s, 3H, OCH 3 ), 0.78 (s, 3H, CH $\left.\mathbf{H}_{3}\right) \mathrm{ppm} .{ }^{13} \mathrm{C}$ NMR (125 $\left.\mathrm{MHz}, \mathrm{CDCl}_{3}\right): \delta 165.7,165.0,160.3,146.54,146.46,143.0,142.3,132.6(2 \mathrm{C}), 130.8(2 \mathrm{C}), 128.8,127.1$, 125.6, 122.7 (2C), 121.2, 120.0, 114.3 (2C), 110.7, 106.6, 92.2, 68.9, 55.4, 52.8, 52.2, 13.2 ppm. IR (KBr): 3456, 2926, 2849, 2361, 2333, 1727, 1599, 1511, 1440, 1342, 1243, 1024, 847, 703, $527 \mathrm{~cm}^{-1}$. HRMS (ESI) m/z: [M+H] ${ }^{+}$calcd for $\mathrm{C}_{28} \mathrm{H}_{25} \mathrm{~N}_{2} \mathrm{O}_{8}$ 517.1611; found 517.1607.

\section{Dimethyl 8a-hydroxy-3'-methyl-1,2-bis(4-nitrophenyl)-3',8a-dihydrocyclopenta[hi]indolizine-7,8- dicarboxylate (3g)}

Reaction of $1 \mathrm{~g}(0.5 \mathrm{~g}, 1.3 \mathrm{mmol})$ with DMAD $(0.25 \mathrm{~mL}, 2.0 \mathrm{mmol})$ as per the protocol described above for the synthesis of $\mathbf{3 a}$ afforded the product $\mathbf{3 g}$ in $40 \%$ yield $(272 \mathrm{mg})$. Orange solid, m.p. $98-100{ }^{\circ} \mathrm{C}$. $R f=0.42(40 \% \mathrm{EtOAc} / \mathrm{Hexane}) .{ }^{1} \mathrm{H}$ NMR $\left(500 \mathrm{MHz}, \mathrm{CDCl}_{3}\right): \delta 8.15(\mathrm{~d}, J=8.25 \mathrm{~Hz}, 2 \mathrm{H}, \mathrm{ArH}), 8.03$ $(\mathrm{d}, J=8.25 \mathrm{~Hz}, 2 \mathrm{H}, \operatorname{ArH}), 7.34(\mathrm{~d}, J=8.32 \mathrm{~Hz}, 2 \mathrm{H}, \operatorname{ArH}), 7.23(\mathrm{~d}, J=8.44 \mathrm{~Hz}, 2 \mathrm{H}, \operatorname{ArH}), 6.71(\mathrm{~d}, J=$ $5.77 \mathrm{~Hz}, 1 \mathrm{H}, \mathrm{CH}), 6.70(\mathrm{~d}, J=6.61 \mathrm{~Hz}, 1 \mathrm{H}, \mathrm{CH}), 6.04(\mathrm{appt}, J=6.00 \mathrm{~Hz}, 1 \mathrm{H}, \mathrm{CH}), 4.46(\mathrm{~s}, 1 \mathrm{H}, \mathrm{OH})$, 3.94 (s, 3H, OCH 3 ), 3.55 (s, 3H, OCH 3 ), 0.84 (s, 3H, CH $\mathbf{C H}_{3}$ ppm. ${ }^{13} \mathrm{C} \mathrm{NMR}\left(125 \mathrm{MHz}, \mathrm{CDCl}_{3}\right): \delta 165.5$, 164.7, 148.3, 147.1, 144.2, 143.8, 140.8, 136.0, 132.5 (2C), 130.5 (2C), 128.2, 128.1, 125.8, 124.1 (2C), 123.0 (2C), 120.1, 111.9, 109.7, 92.2, 69.2, 52.9, 52.4, 13.4 ppm. IR (KBr): 3479, 2953, 2921, 2860 , 1728, 1593, 1519, 1346, 1314, 1234, 1199, 1104, 1062, 1010, 855, 743, $514 \mathrm{~cm}^{-1}$. HRMS (ESI) m/z: $[\mathrm{M}+\mathrm{H}]^{+}$calcd for $\mathrm{C}_{27} \mathrm{H}_{22} \mathrm{~N}_{3} \mathrm{O}_{9} 532.1356$; found 532.1340.

\section{$\mathrm{HBF}_{4}$. OEt 2 -assisted aromatization of 3a-g}
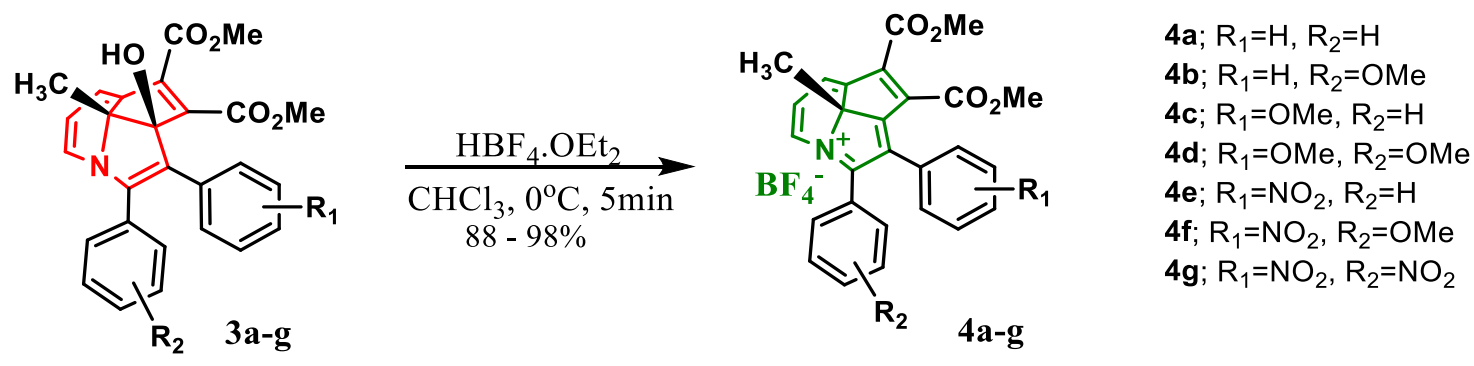

Typical procedure: $\mathrm{HBF}_{4} . \mathrm{OEt}_{2}(0.14 \mathrm{~mL}, 1.0 \mathrm{mmol} ; 1.5$ equiv. with respect to $3 \mathrm{a})$ was added-drop wise to a stirred solution of $\mathbf{3 a}(0.3 \mathrm{~g}, 0.68 \mathrm{mmol})$ in chloroform at $0^{\circ} \mathrm{C}$ under nitrogen atmosphere. The reaction mixture was allowed to stir at the same temperature for 5 minutes and subsequently concentrated under reduced pressure. The crude product was subjected to repeated washings with diethyl ether $(15 \mathrm{~mL}$ x 3$)$, followed by hexane $(15 \mathrm{~mL} \times 3)$ to get the product $4 \mathbf{a}$ in pure form. The same protocol was repeated with $\mathbf{3 b} \mathbf{b}$ g to get the corresponding products $\mathbf{4 b - g}$.

Note: Of the compounds reported here, all except $\mathbf{4 e}, \mathbf{4 g}$ and $\mathbf{4 f}$ are solids, and were purified by repeated washing with diethyl ether, followed by hexane as mentioned above. 
Compounds $4 \mathbf{e}, \mathbf{4 g}$ and $\mathbf{4 f}$ on the other hand are gummy solids, which were found to retain slight amount of $\mathrm{HBF}_{4} \cdot \mathrm{E}_{\mathrm{t}} \mathrm{O}$ even after repeated washings. Two additional signals in their NMR are from this residual $\mathrm{HBF}_{4} \cdot \mathrm{E}_{\mathrm{t} 2} \mathrm{O}$. We have marked these extra peaks in their NMR, and also have included the spectrum of a commercial sample of $\mathrm{HBF}_{4}$. OEt 2 (Figure S35-36) to indicate this.

\section{Compound 4a}

96\% yield; Yellow solid, m.p. $158-160{ }^{\circ} \mathrm{C} ;{ }^{1} \mathrm{H}$ NMR (500 MHz, $\left.\mathrm{CDCl}_{3}\right): \delta 9.48(\mathrm{~d}, J=6.10 \mathrm{~Hz}, 1 \mathrm{H}$, CH), $8.54(\mathrm{~d}, J=6.95 \mathrm{~Hz}, 1 \mathrm{H}, \mathrm{CH}), 8.34$ (appt, $J=6.17 \mathrm{~Hz}, 1 \mathrm{H}, \mathrm{CH}), 7.74(\mathrm{~d}, J=7.30 \mathrm{~Hz}, 2 \mathrm{H}, \operatorname{ArH})$, $7.69-7.72(\mathrm{~m}, 1 \mathrm{H}, \operatorname{ArH}), 7.63-7.66(\mathrm{~m}, 2 \mathrm{H}, \operatorname{ArH}), 7.49-7.52(\mathrm{~m}, 1 \mathrm{H}, \operatorname{ArH}), 7.41$ (appt, $J=7.65 \mathrm{~Hz}$, $2 \mathrm{H}, \operatorname{ArH}), 7.33$ (d, J=7.65 Hz, 2H, ArH), 4.10 (s, 3H, OCH 3 ), 3.66 (s, 3H, OCH 3 ), $-0.23\left(\mathrm{~s}, 3 \mathrm{H}, \mathrm{CH}_{3}\right)$ ppm. ${ }^{13} \mathrm{C}$ NMR (125 MHz, $\left.\mathrm{CDCl}_{3}\right): \delta 174.4,164.1,163.1,161.5,151.4,145.8,140.8,140.5134 .3$, 134.2, 131.1, 130.7 (2C), 130.42 (2C), 130.35 (2C), 129.4, 129.3, 129.2 (2C), 126.1, 126.0, 73.1, 53.9, 53.3, 29.3 ppm. IR (ATR): 3077, 3010, 1727, 1430, 1253, 1060, $740 \mathrm{~cm}^{-1}$. HRMS (ESI) m/z: [M] $]^{+}$calcd for $\mathrm{C}_{27} \mathrm{H}_{22} \mathrm{NO}_{4} 424.1549$; found 424.1534 .

\section{$\underline{\text { Compound 4b }}$}

$88 \%$ yield; Orange solid, m.p. $154-156{ }^{\circ} \mathrm{C} ;{ }^{1} \mathrm{H}$ NMR (500 MHz, $\left.\mathrm{CDCl}_{3}\right): \delta 9.48(\mathrm{~d}, J=6.52 \mathrm{~Hz}, 1 \mathrm{H}$, CH), 8.40 (d, $J=6.83 \mathrm{~Hz}, 1 \mathrm{H}, \mathrm{CH}), 8.27$ (appt, $J=6.69 \mathrm{~Hz}, 1 \mathrm{H}, \mathrm{CH}), 7.75(\mathrm{~d}, J=8.69 \mathrm{~Hz}, 2 \mathrm{H}, \operatorname{ArH})$, $7.49-7.52$ (m, 1H, ArH), $7.41-7.44$ (m, 2H, ArH), 7.33 (d, J=7.30 Hz, 2H, ArH), 7.12 (d, J= 8.85

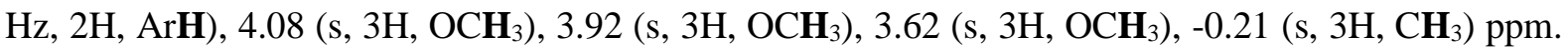
${ }^{13} \mathrm{C}$ NMR $\left(125 \mathrm{MHz}, \mathrm{CDCl}_{3}\right): \delta 173.9,164.8,164.5,163.2,161.6,150.0,145.4,140.5,140.3,133.5$, 133.1 (2C), 131.1, 130.5 (2C), 129.8, 129.2 (2C), 128.6, 124.9, 118.3, 116.1 (2C), 72.9, 56.1, 53.8, 53.3, 28.7 ppm. IR (ATR): 3066, 2977, 1731, 1600, 1434, 1261, 1056, $725 \mathrm{~cm}^{-1}$. HRMS (ESI) m/z: $[\mathrm{M}]^{+}$calcd for $\mathrm{C}_{28} \mathrm{H}_{24} \mathrm{NO}_{5} 454.1654$; found 454.1637 .

\section{Compound $4 \mathrm{c}$}

$94 \%$ yield; Red solid, m.p. $56-58{ }^{\circ} \mathrm{C} ;{ }^{1} \mathrm{H}$ NMR $\left(500 \mathrm{MHz}, \mathrm{CDCl}_{3}\right): \delta 9.36(\mathrm{~d}, J=6.56 \mathrm{~Hz}, 1 \mathrm{H}, \mathrm{CH})$, 8.50 (d, $J=7.09 \mathrm{~Hz}, 1 \mathrm{H}, \mathrm{CH}), 8.29$ (appt, $J=6.88 \mathrm{~Hz}, 1 \mathrm{H}, \mathbf{C H}), 7.69-7.73(\mathrm{~m}, 3 \mathrm{H}, \mathrm{ArH}), 7.63-7.66$ $(\mathrm{m}, 2 \mathrm{H}, \operatorname{ArH}), 7.27(\mathrm{~d}, J=8.86 \mathrm{~Hz}, 2 \mathrm{H}, \operatorname{ArH}), 6.90(\mathrm{~d}, J=8.86 \mathrm{~Hz}, 2 \mathrm{H}, \operatorname{ArH}), 4.07\left(\mathrm{~s}, 3 \mathrm{H}, \mathrm{OCH}_{3}\right)$, 3.84 (s, 3H, OCH $\left.\mathbf{O H}_{3}\right), 3.73$ (s, 3H, $\mathrm{OCH}_{3}$ ), -0.27 (s, 3H, $\left.\mathrm{CH}_{3}\right)$ ppm. ${ }^{13} \mathrm{C} \mathrm{NMR}\left(125 \mathrm{MHz}, \mathrm{CDCl}_{3}\right): \delta 173.0$, 163.5, 162.4, 162.2, 161.6, 151.5, 144.5, 141.2, 141.1, 134.1, 133.6, 132.5 (2C), 130.4 (2C), 130.2 (2C), 129.3, 126.4, 125.4, 121.7, 114.8 (2C), 73.1, 55.7, 53.8, 53.5, 29.3 ppm. IR (ATR): 3089, 3000, 1721, 1604, 1508, 1438, 1257, 1056, 840, $736 \mathrm{~cm}^{-1}$. HRMS (ESI) m/z: [M] ${ }^{+}$calcd for $\mathrm{C}_{28} \mathrm{H}_{24} \mathrm{NO}_{5} 454.1654$; found 454.1636 .

\section{Compound 4d}

98\% yield; Red solid, m.p. $176-178{ }^{\circ} \mathrm{C} ;{ }^{1} \mathrm{H}$ NMR $\left(500 \mathrm{MHz}, \mathrm{CDCl}_{3}\right): \delta 9.43(\mathrm{~d}, J=6.46 \mathrm{~Hz}, 1 \mathrm{H}, \mathrm{CH})$, $8.37(\mathrm{~d}, J=6.94 \mathrm{~Hz}, 1 \mathrm{H}, \mathrm{CH}), 8.27$ (appt, $J=6.76 \mathrm{~Hz}, 1 \mathrm{H}, \mathrm{CH}), 7.76(\mathrm{~d}, J=8.86 \mathrm{~Hz}, 2 \mathrm{H}, \operatorname{ArH}), 7.28$ $(\mathrm{d}, J=8.86 \mathrm{~Hz}, 2 \mathrm{H}, \operatorname{ArH}), 7.15(\mathrm{~d}, J=8.87 \mathrm{~Hz}, 2 \mathrm{H}, \operatorname{ArH}), 6.93(\mathrm{~d}, J=8.77 \mathrm{~Hz}, 2 \mathrm{H}, \operatorname{ArH}), 4.07(\mathrm{~s}, 3 \mathrm{H}$, $\left.\mathrm{OCH}_{3}\right), 3.93$ (s, 3H, OCH 3 ), 3.86 (s, 3H, OCH 3 ), 3.71 (s, 3H, OCH 3 ), -0.23 (s, 3H, CH 3$)$ ppm. ${ }^{13} \mathrm{C} \mathrm{NMR}$ (125 MHz, $\left.\mathrm{CDCl}_{3}\right): \delta 172.6,164.6,163.6,163.0,162.1,161.7,150.2,144.1,141.1,140.9,132.8(2 \mathrm{C})$, 132.7, 132.3 (2C), 128.6, 124.3, 122.0, 118.5, 116.1 (2C), 114.8 (2C), 72.9, 56.1, 55.7, 53.8, 53.4, 28.7 ppm. IR (ATR): 2915, 2854, 1731, 1604, 1508, 1442, 1261, 1184, 1060, $840 \mathrm{~cm}^{-1}$. HRMS (ESI) m/z: $[\mathrm{M}]^{+}$calcd for $\mathrm{C}_{29} \mathrm{H}_{26} \mathrm{NO}_{6} 484.1760$; found 484.1735 . 


\section{Compound 4e}

90\% yield; Yellow gummy solid; ${ }^{1} \mathrm{H}$ NMR $\left(500 \mathrm{MHz}, \mathrm{CDCl}_{3}\right): \delta 9.32(\mathrm{~d}, J=6.49 \mathrm{~Hz}, 1 \mathrm{H}, \mathrm{CH}), 8.51$ $(\mathrm{d}, J=6.98 \mathrm{~Hz}, 1 \mathrm{H}, \mathrm{CH}), 8.18-8.21(\mathrm{~m}, 3 \mathrm{H}, \mathrm{CH}+\mathrm{ArH}), 7.65-7.68(\mathrm{~m}, 3 \mathrm{H}, \mathrm{ArH}), 7.55-7.59(\mathrm{~m}, 4 \mathrm{H}$, $\mathrm{ArH}), 4.06$ (s, 3H, OCH $\left.{ }_{3}\right), 3.68$ (s, $\left.3 \mathrm{H}, \mathrm{OCH}_{3}\right),-0.24$ (s, 3H, $\left.\mathrm{CH}_{3}\right) \mathrm{ppm} .{ }^{13} \mathrm{C} \mathrm{NMR}\left(125 \mathrm{MHz}, \mathrm{CDCl}_{3}\right)$ : $\delta 175.3,165.6,162.5,161.6,151.2,148.8,147.8,139.0,137.9,135.9,134.5,134.3,132.2(2 \mathrm{C}), 130.6$ (2C), 130.4 (2C), 128.9, 126.7, 125.7, 123.8 (2C), 73.4, 54.0, 53.5, 29.3 ppm. IR (ATR): 3093, 2981, $1731,1608,1519,1442,1353,1257,1064,860,725 \mathrm{~cm}^{-1}$. HRMS (ESI) m/z: [M] calcd for $\mathrm{C}_{27} \mathrm{H}_{21} \mathrm{~N}_{2} \mathrm{O}_{6}$ 469.1400; found 469.1379 .

\section{Compound 4 f}

97\% yield; Red gummy solid; ${ }^{1} \mathrm{H}$ NMR (500 MHz, $\left.\mathrm{CDCl}_{3}\right): \delta 9.33(\mathrm{~d}, J=6.54 \mathrm{~Hz}, 1 \mathrm{H}, \mathrm{CH}), 8.42$ (d, $J$ $=6.54 \mathrm{~Hz}, 1 \mathrm{H}, \mathrm{CH}), 8.25(\mathrm{~d}, J=8.26 \mathrm{~Hz}, 2 \mathrm{H}, \mathrm{ArH}), 8.17$ (appt, $J=6.54 \mathrm{~Hz}, 1 \mathrm{H}, \mathrm{CH}), 7.66$ (d, $J=9.00$ $\mathrm{Hz}, 2 \mathrm{H}, \operatorname{ArH}), 7.57(\mathrm{~d}, J=8.14 \mathrm{~Hz}, 2 \mathrm{H}, \operatorname{ArH}), 7.09$ (d, $J=9.00 \mathrm{~Hz}, 2 \mathrm{H}, \operatorname{ArH}), 4.08\left(\mathrm{~s}, 3 \mathrm{H}, \mathrm{OCH}_{3}\right)$,

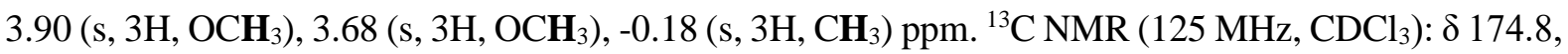
$165.9,164.8,162.7,161.7,149.9,148.8,147.5,138.8,137.5,136.3,133.7,133.1$ (2C), 132.0 (2C), 128.3, 125.7, 123.9 (2C), 117.8, 116.1 (2C), 73.3, 56.1, 54.0, 53.5, 28.7 ppm. IR (ATR): 3097, 2969, 1731, 1604, 1523, 1438, 1357, 1257, 1056, 852, $744 \mathrm{~cm}^{-1}$. HRMS (ESI) m/z: [M] falcd for $\mathrm{C}_{28} \mathrm{H}_{23} \mathrm{~N}_{2} \mathrm{O}_{7}$ 499.1505; found 499.1487 .

\section{Compound 4g}

92\% yield; Brown gummy solid; ${ }^{1} \mathrm{H}$ NMR (500 MHz, $\left.\mathrm{CD}_{3} \mathrm{CN}\right): \delta 9.26(\mathrm{~d}, J=6.54 \mathrm{~Hz}, 1 \mathrm{H}, \mathrm{CH}), 8.65$ $(\mathrm{d}, J=7.07 \mathrm{~Hz}, 1 \mathrm{H}, \mathrm{CH}), 8.41(\mathrm{~d}, J=8.88 \mathrm{~Hz}, 2 \mathrm{H}, \operatorname{ArH}), 8.25(\mathrm{~d}, J=8.83 \mathrm{~Hz}, 2 \mathrm{H}, \operatorname{ArH}), 8.22(\mathrm{~d}, J=$ $6.75 \mathrm{~Hz}, 1 \mathrm{H}, \mathrm{CH} 7.86$ (d, J = 8.92 Hz, 2H, ArH), 7.56 (d, $J=8.92 \mathrm{~Hz}, 2 \mathrm{H}, \operatorname{ArH}), 4.08\left(\mathrm{~s}, 3 \mathrm{H}, \mathrm{OCH}_{3}\right)$, 3.67 (s, 3H, OCH $),-0.24$ (s, 3H, CH 3 ) ppm. $\left.{ }^{13} \mathrm{C} \mathrm{NMR} \mathrm{(125} \mathrm{MHz,} \mathrm{CD}_{3} \mathrm{CN}\right): \delta 177.2,163.5,163.4$, $162.7,153.7,151.6,150.1,149.5,140.3,139.5,136.4,136.3,133.1$ (2C), 133.0 (2C), 132.6, 129.8, 127.7, 125.8 (2C), 124.9 (2C), 74.6, 54.6, 54.2, 29.2 ppm. IR (ATR): 3004, 1635, 1434, 1041, $917 \mathrm{~cm}^{-}$ ${ }^{1}$. HRMS (ESI) m/z: [M] calcd for $\mathrm{C}_{27} \mathrm{H}_{20} \mathrm{~N}_{3} \mathrm{O}_{8}$ 514.1250; found 514.1224.

\section{Gram scale synthesis of compound $4 d$}

$\mathrm{HBF}_{4} . \mathrm{OEt}_{2}(0.4 \mathrm{~mL}, 3.0 \mathrm{mmol} ; 1.5$ equiv. with respect to $\mathbf{3 d})$ was added-drop wise to a stirred solution of $\mathbf{3 d}(1 \mathrm{~g}, 2.0 \mathrm{mmol})$ in chloroform at $0^{\circ} \mathrm{C}$ under nitrogen atmosphere. The reaction mixture was allowed to stir at the same temperature for 5 minutes and subsequently concentrated under reduced pressure. The crude product was subjected to repeated washings with diethyl ether $(50 \mathrm{~mL} \times 3)$, followed by hexane $(50 \mathrm{~mL} \times 3)$ to get the product $4 \mathbf{d}$ in pure form $(1.1 \mathrm{~g}, 1.93 \mathrm{mmol}, 97 \%$ yield $)$. 
a)

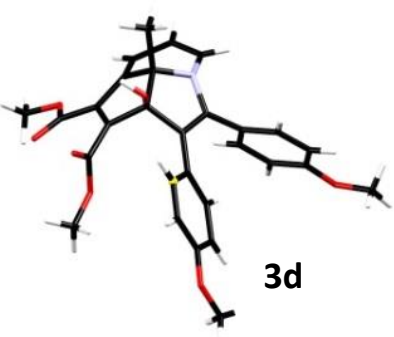

c)

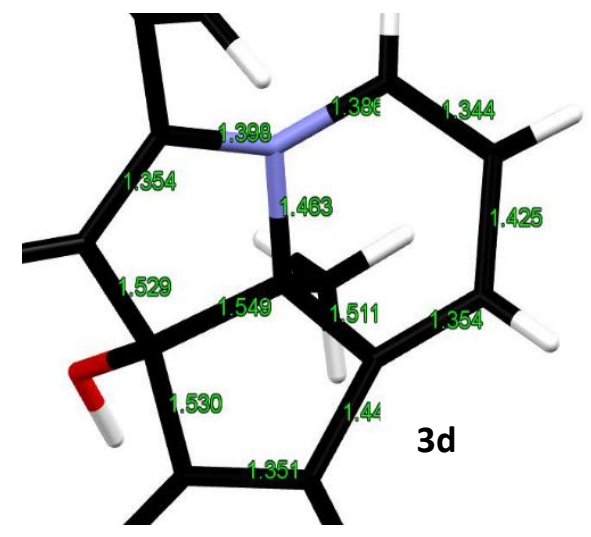

b)

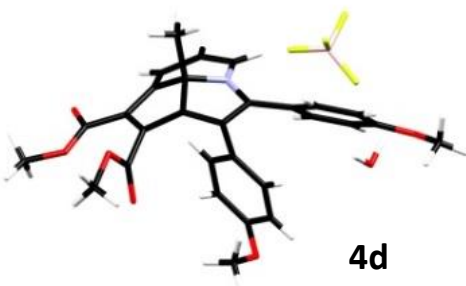

d)

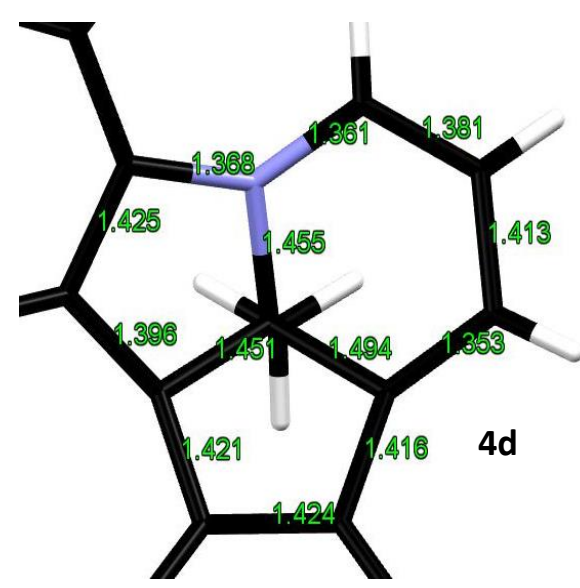

e)

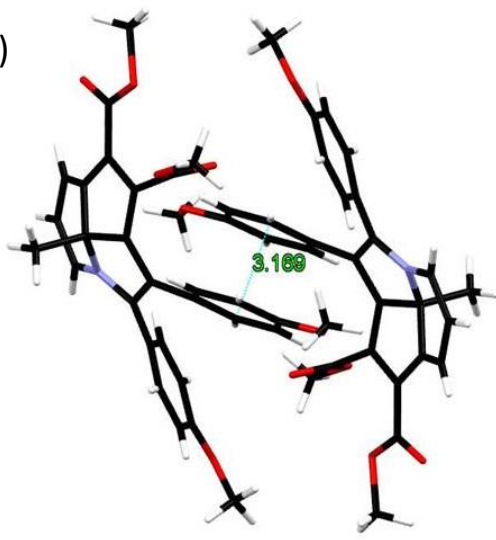

f)

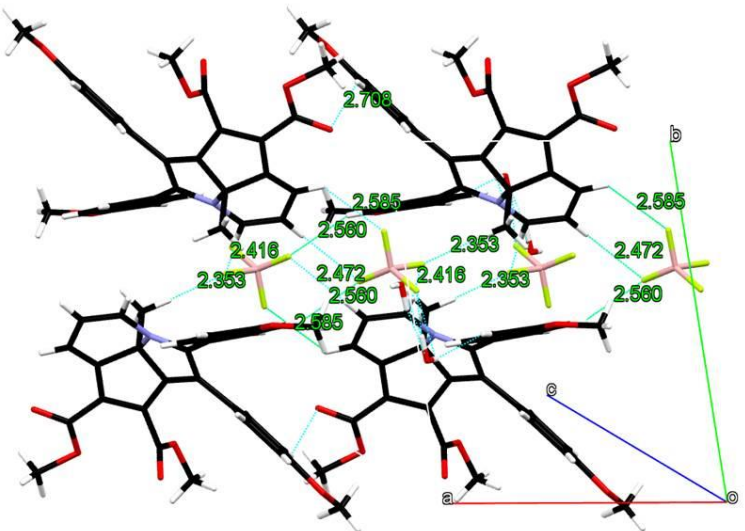

Figure S1. a) X-ray structure of 3d; b) X-ray structure of 4d; c\&d) bond length between the atoms which constitute the tricyclic moiety in $\mathbf{3 d}$ and $\mathbf{4 d}$; e) 'parallelly displaced' arrangement of C-3 aryl rings of enantiomers of $\mathbf{4 d}$ with centroid-centroid distance of $3.17 \AA$ (offset distance $\sim 2.7 \AA$ ); f) secondary interactions involving enantiomers of $\mathbf{4 d}$ with the counter ions and cocrystallized water molecules. CCDC numbers of 3d and 4d are 2043141 and 2043140 respectively. 
Table S1. Crystallographic data of compound $\mathbf{3 d}$

\begin{tabular}{|c|c|c|c|}
\hline Bond precision: & $\mathrm{C}-\mathrm{C}=0.0049 \mathrm{~A}$ & Wavelength $=$ & 0.71073 \\
\hline \multirow[t]{2}{*}{ Cell: } & $a=13.3116(8)$ & $\mathrm{b}=19.6751(11)$ & $C=9.5416(5)$ \\
\hline & alpha $=90$ & bet $a=98.108(2)$ & gamma $=90$ \\
\hline \multirow[t]{2}{*}{ Temperature: } & $296 \mathrm{~K}$ & & \\
\hline & Calculated & Reported & \\
\hline Volume & $2474.0(2)$ & $2474.0(2)$ & \\
\hline Space group & P $21 / \mathrm{C}$ & P $21 / \mathrm{C}$ & \\
\hline Hall group & $-\mathrm{P} 2 \mathrm{ybc}$ & $-\mathrm{P} 2 \mathrm{ybc}$ & \\
\hline Moiety formula & $\begin{array}{llll}\mathrm{C} 29 & \mathrm{H} 27 & \mathrm{~N} & \mathrm{O}\end{array}$ & $?$ & \\
\hline Sum formula & $\begin{array}{llll}\mathrm{C} 29 & \mathrm{H} 27 & \mathrm{~N} & \mathrm{O}\end{array}$ & $\mathrm{C} 29 \mathrm{H} 27 \mathrm{~N}$ & 07 \\
\hline $\mathrm{Mr}$ & 501.52 & 501.51 & \\
\hline $\mathrm{Dx}, \mathrm{g} \mathrm{cm}-3$ & 1.347 & 1.346 & \\
\hline $\mathrm{z}$ & 4 & 4 & \\
\hline $\mathrm{Mu} \quad(\mathrm{mm}-1)$ & 0.097 & 0.097 & \\
\hline F000 & 1056.0 & 1056.0 & \\
\hline $\mathrm{F} 000^{\prime}$ & 1056.57 & & \\
\hline $\mathrm{h}, \mathrm{k}, \mathrm{lmax}$ & $15,22,11$ & $15,22,11$ & \\
\hline Nref & 4005 & 3658 & \\
\hline$T \min , T \max$ & $0.976,0.990$ & & \\
\hline Tmin' & 0.976 & & \\
\hline \multicolumn{4}{|c|}{ Correction method= Not given } \\
\hline Data completenes & $s s=0.913$ & Theta $(\max )=24.260$ & \\
\hline$R$ (reflections) $=$ & $0.0567(2525)$ & wR2 (reflections) = & $0.1402(3658)$ \\
\hline$S=1.076$ & Npar $=$ & 344 & \\
\hline
\end{tabular}

Crystals of $\mathbf{3 d}$ were grown by slow evaporation of its solution in methanol 


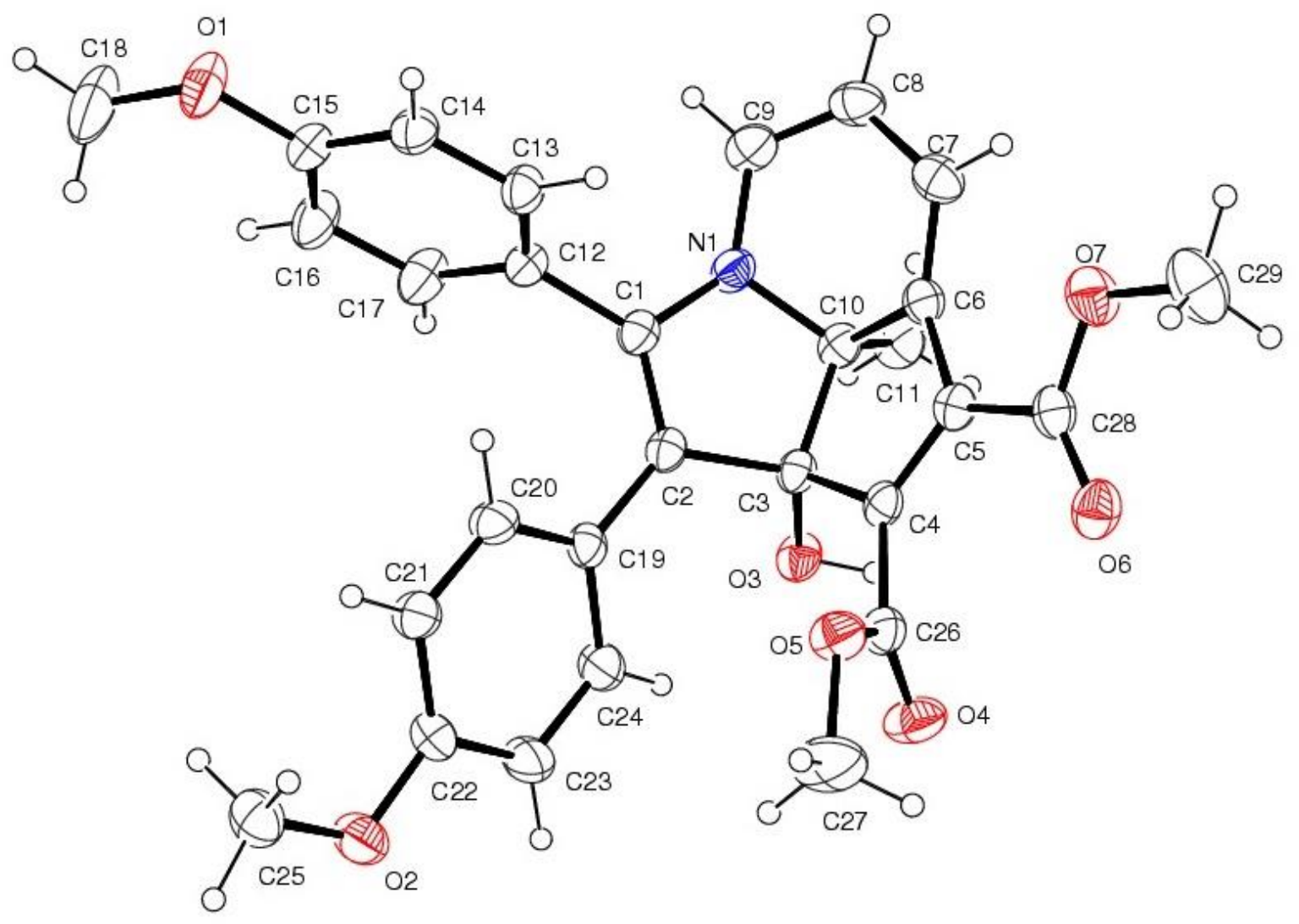

Figure S2. ORTEP picture of compound 3d (30\% probability level) 
Table S2. Crystallographic data of compound $\mathbf{4 d}$

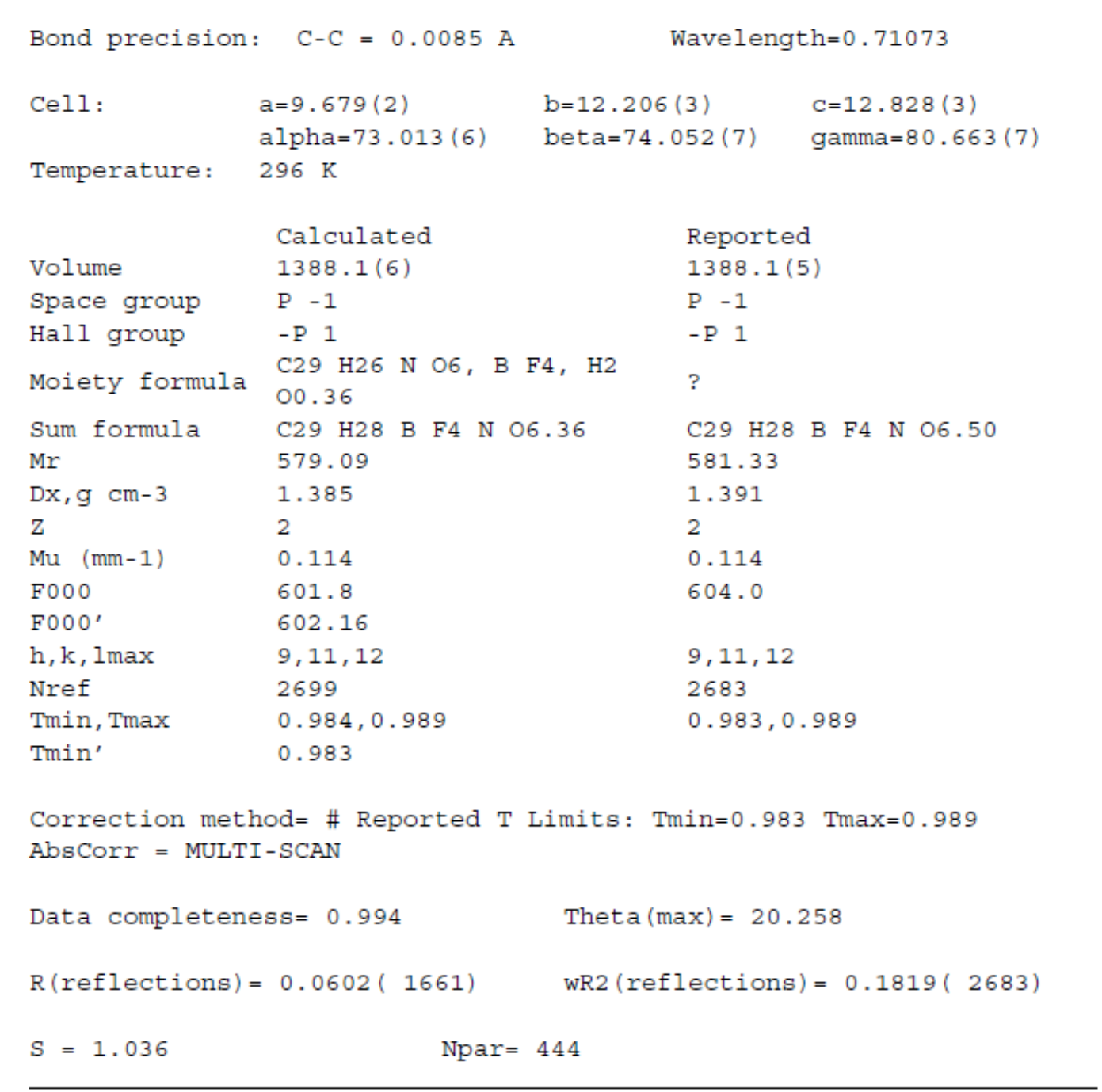

Crystals of $\mathbf{4 d}$ were grown by slow evaporation of its solution in $\mathrm{CDCl}_{3}$. 

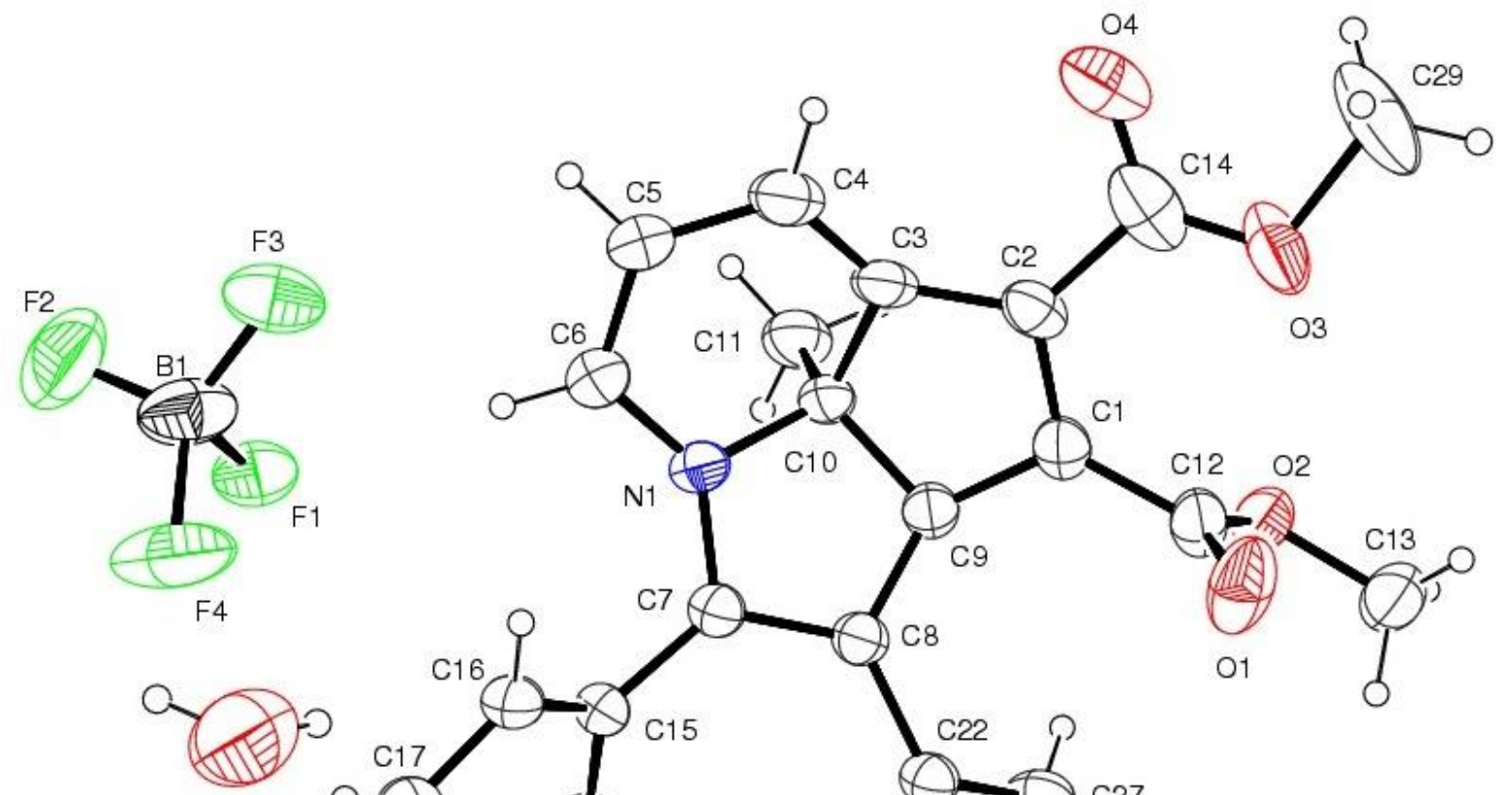

$\mathrm{O} 7$
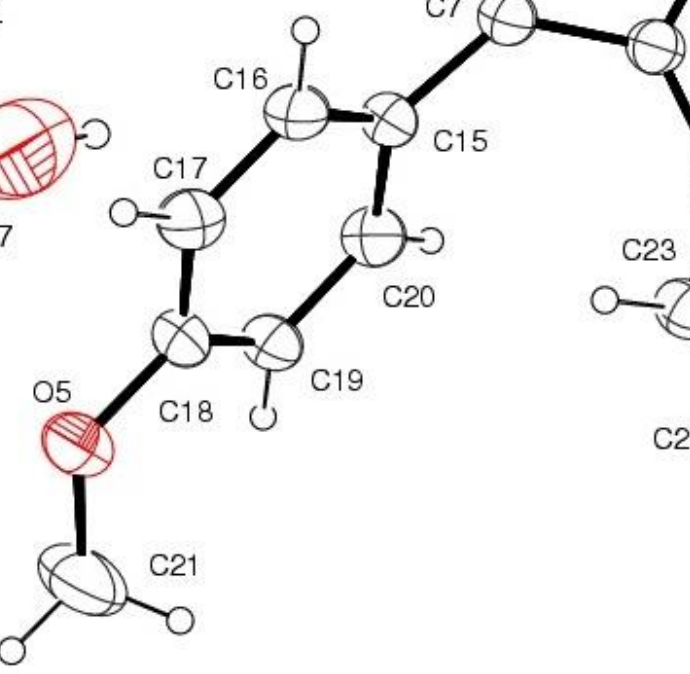

C22

C27

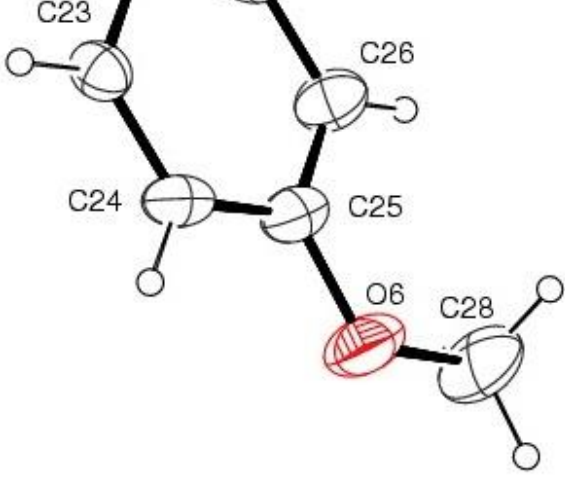

Figure S3. ORTEP picture of compound $\mathbf{4 d}$ (30\% probability level) 

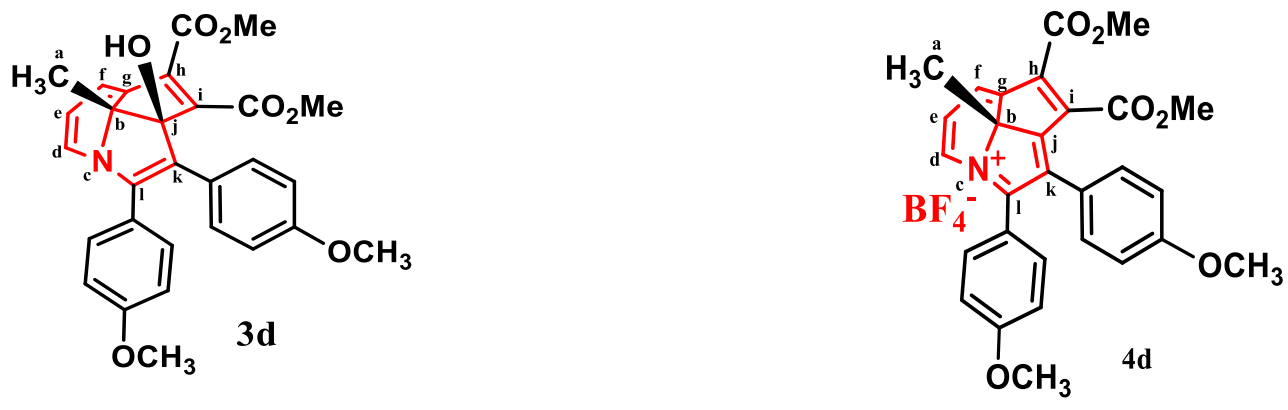

Table S3. Comparison of bond angles in $\mathbf{3 d}$ and $\mathbf{4 d}$ (from diffraction data)

\begin{tabular}{|l|l|l|l|}
\hline Sl.No & Angle & \multicolumn{1}{|c|}{ 3d } & 4d \\
\hline 1. & $\angle \mathrm{abc}$ & $111.8^{\circ}$ & $109.6^{\circ}$ \\
\hline 2. & $\angle \mathrm{abg}$ & $111.8^{\circ}$ & $109.0^{\circ}$ \\
\hline 3 & $\angle \mathrm{abj}$ & $114.8^{\circ}$ & $114.4^{\circ}$ \\
\hline 4. & $\angle \mathrm{cbg}$ & $107.2^{\circ}$ & $114.2^{\circ}$ \\
\hline 5. & $\angle \mathrm{cbj}$ & $105.2^{\circ}$ & $103.9^{\circ}$ \\
\hline 6. & $\angle \mathrm{gbj}$ & $105.5^{\circ}$ & $105.9^{\circ}$ \\
\hline 7. & $\angle \mathrm{bjk}$ & $103.2^{\circ}$ & $108.1^{\circ}$ \\
\hline 8. & $\angle \mathrm{bji}$ & $102.6^{\circ}$ & $106.7^{\circ}$ \\
\hline 9. & $\angle \mathrm{ijk}$ & $113.1^{\circ}$ & $140.3^{\circ}$ \\
\hline
\end{tabular}

Table S4. Change in bond lengths of the central ring as $\mathbf{3 d}$ transforms to $\mathbf{4 d}$ (from diffraction data)

\begin{tabular}{|l|l|l|l|}
\hline Sl.No & \multicolumn{1}{|c|}{ Bond } & $\begin{array}{l}\text { 3d Bond } \\
\text { Length }(\AA ̊)\end{array}$ & $\begin{array}{l}\text { 4d Bond } \\
\text { length }(\AA)\end{array}$ \\
\hline 1. & $\mathrm{C}_{\mathrm{a}}-\mathrm{C}_{\mathrm{b}}$ & 1.528 & 1.540 \\
\hline 2. & $\mathrm{C}_{\mathrm{b}}-\mathrm{C}_{\mathrm{c}}$ & 1.463 & 1.455 \\
\hline 3. & $\mathrm{C}_{\mathrm{b}}-\mathrm{C}_{\mathrm{j}}$ & 1.549 & 1.451 \\
\hline 4. & $\mathrm{C}_{\mathrm{b}}-\mathrm{C}_{\mathrm{g}}$ & 1.511 & 1.494 \\
\hline 5. & $\mathrm{~N}_{\mathrm{c}}-\mathrm{C}_{\mathrm{l}}$ & 1.398 & 1.368 \\
\hline 6. & $\mathrm{C}_{\mathrm{c}}-\mathrm{C}_{\mathrm{k}}$ & 1.354 & 1.425 \\
\hline 7. & $\mathrm{C}_{\mathrm{k}}-\mathrm{C}_{\mathrm{j}}$ & 1.529 & 1.396 \\
\hline 8. & $\mathrm{C}_{\mathrm{j}}-\mathrm{C}_{\mathrm{i}}$ & 1.530 & 1.421 \\
\hline 9. & $\mathrm{C}_{\mathrm{i}}-\mathrm{C}_{\mathrm{h}}$ & 1.351 & 1.424 \\
\hline 10. & $\mathrm{C}_{\mathrm{h}}-\mathrm{C}_{\mathrm{g}}$ & 1.448 & 1.416 \\
\hline 11. & $\mathrm{C}_{\mathrm{g}}-\mathrm{C}_{\mathrm{f}}$ & 1.354 & 1.353 \\
\hline 12. & $\mathrm{C}_{\mathrm{f}}-\mathrm{C}_{\mathrm{e}}$ & 1.425 & 1.413 \\
\hline 13. & $\mathrm{C}_{\mathrm{e}}-\mathrm{C}_{\mathrm{d}}$ & 1.344 & 1.380 \\
\hline 14. & $\mathrm{C}_{\mathrm{d}}-\mathrm{C}_{\mathrm{c}}$ & 1.386 & 1.361 \\
\hline
\end{tabular}




\section{Photophysical characterization}

Absorption spectra were recorded on Shimadzu UV 2450 instrument, and fluorescence spectra were recorded using a Perkin Elmer LS-45 fluorescence spectrometer. The slit-width was adjusted during both excitation and emission measurements using appropriate filters. The absolute solid-state fluorescence quantum efficiency was measured using Quanta- $\phi$ Horiba instrument coupled with Fluorolog spectrophotometer. Fluorocube - Life Time System JOBINVYON M/S instrument was employed for the time-resolved luminescence decay measurements. A diode laser $\left(\lambda_{\mathrm{ex}}=390 \mathrm{~nm}\right)$ was used as an excitation source. The fluorescence decay profiles were collected near to the respective emission maxima, followed by its analysis with IBH DAS6.

Preparation of samples for UV-Visible and fluorescence spectroscopy: $10^{-2} \mathrm{M}$ stock solutions of the compounds 4a-d were prepared by dissolving the required amount in $10 \mathrm{~mL}$ acetonitrile. $3 \mathrm{~mL}$ of $3.33 \times 10^{-5} \mathrm{M}$ solutions of the respective compounds were prepared in toluene, DCM, EtOAc and $\mathrm{CH}_{3} \mathrm{CN}$ by diluting $10 \mu \mathrm{L}$ of this stock solution to $3 \mathrm{~mL}$ using the respective solvents.

a)

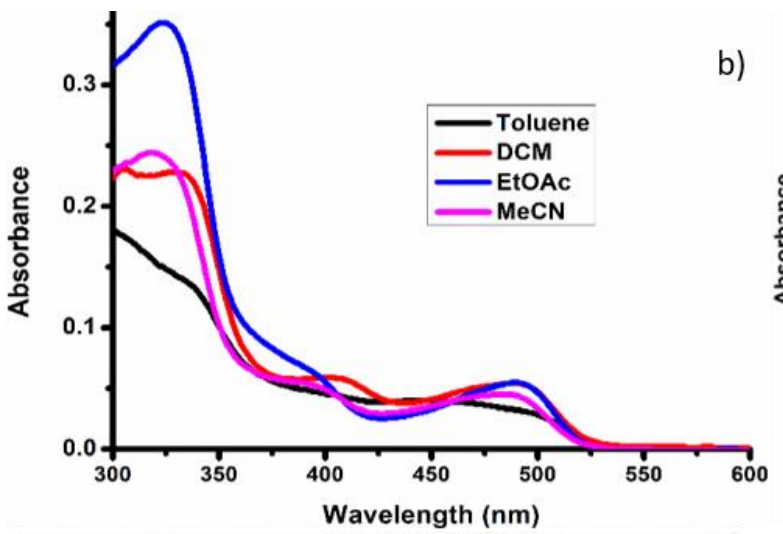

c)

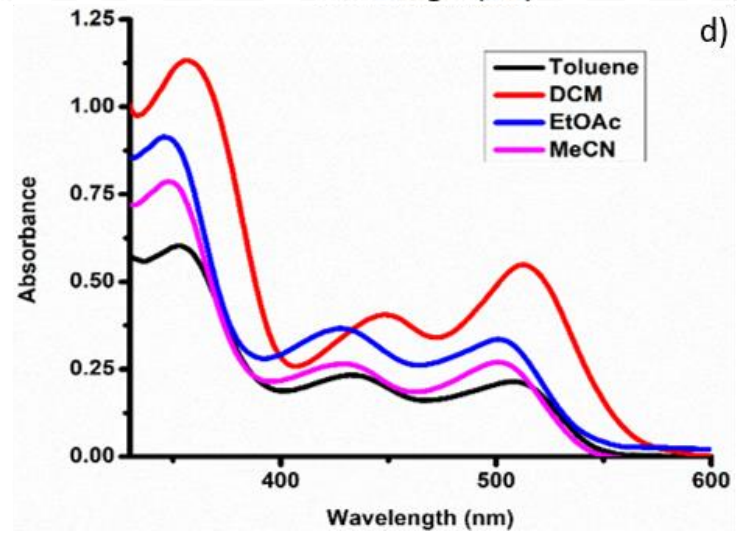

b)

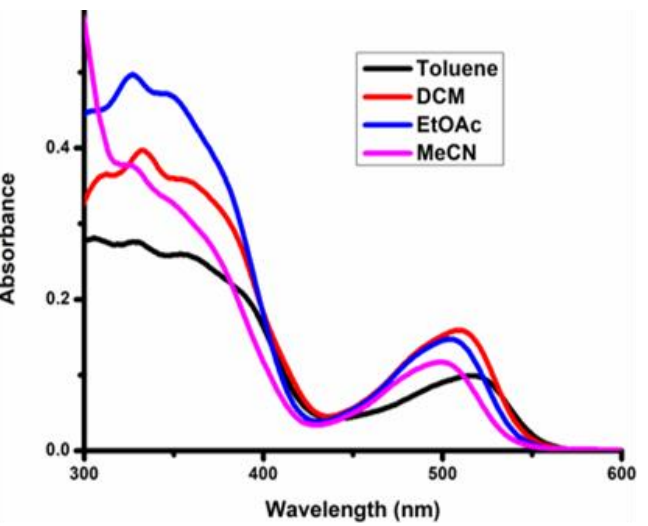

d)

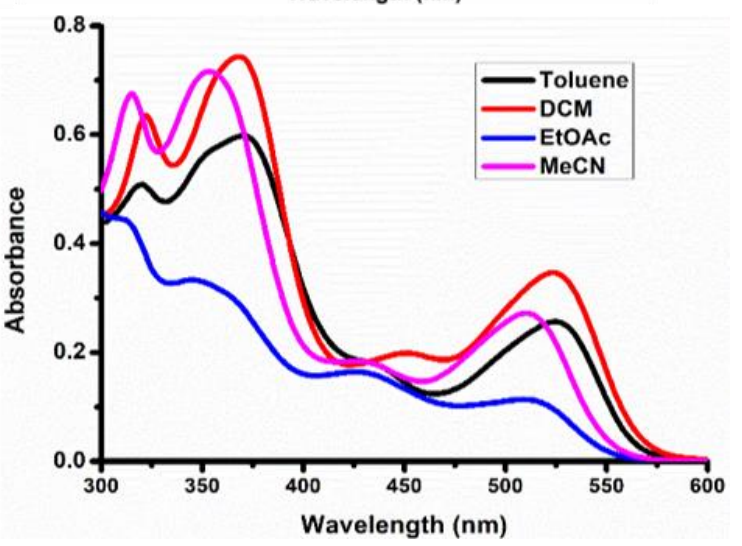

Figure S4. Absorption spectra of $3.33 \times 10^{-5} \mathrm{M}$ solution of a) $\mathbf{4 a}$, b) $\mathbf{4 b}$, c) $\mathbf{4 c}$ and d) $\mathbf{4 d}$ in different solvents. 

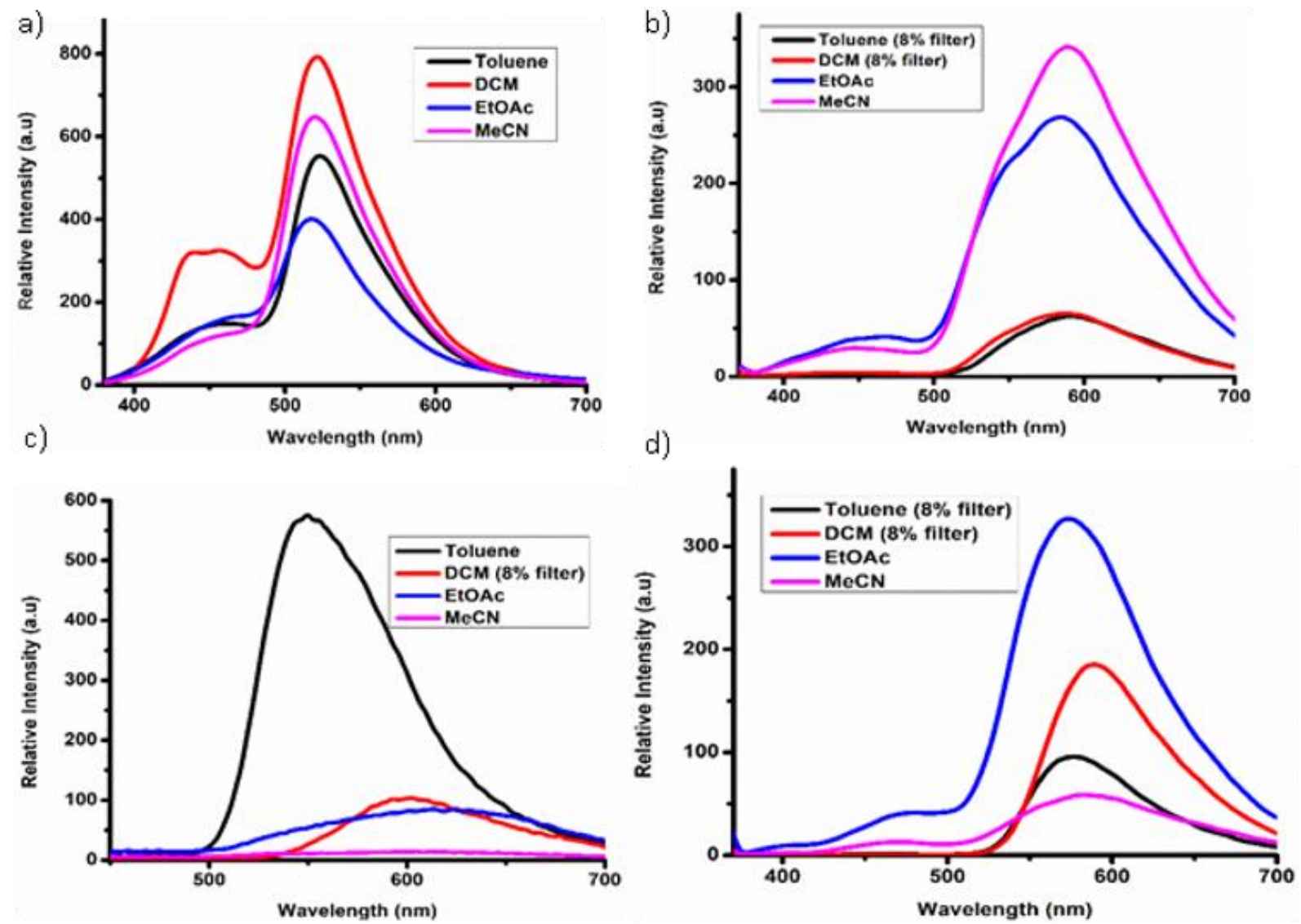

Figure S5. Emission spectra of $3.33 \times 10^{-5} \mathrm{M}$ solution of a) $\mathbf{4 a}$, b) $\mathbf{4 b}$, c) $\mathbf{4 c}$ and d) $\mathbf{4 d}$ in toluene, DCM, EtOAc and $\mathrm{MeCN}$.

Table S5. Absorption and emission maxima of compounds 4a-d in different solvents

\begin{tabular}{|c|c|c|c|c|c|c|c|c|}
\hline \multirow{2}{*}{ Solvent } & \multicolumn{3}{|c|}{ 4a } & \multicolumn{2}{c|}{ 4b } & \multicolumn{2}{c|}{$\mathbf{4 c}$} & \multicolumn{2}{c|}{ 4d } \\
\cline { 2 - 9 } & $\begin{array}{c}\text { Absorption } \\
\text { maxima }\end{array}$ & $\begin{array}{c}\text { Emission } \\
\text { maxima }\end{array}$ & $\begin{array}{c}\text { Absorption } \\
\text { maxima }\end{array}$ & $\begin{array}{c}\text { Emission } \\
\text { maxima }\end{array}$ & $\begin{array}{c}\text { Absorption } \\
\text { maxima }\end{array}$ & $\begin{array}{c}\text { Emission } \\
\text { maxima }\end{array}$ & $\begin{array}{c}\text { Absorption } \\
\text { maxima }\end{array}$ & $\begin{array}{c}\text { Emission } \\
\text { maxima }\end{array}$ \\
\hline Toluene & $\begin{array}{c}490,397, \\
338\end{array}$ & 522,455 & $\begin{array}{c}520,358, \\
329\end{array}$ & 591,442 & $\begin{array}{c}509,433, \\
352\end{array}$ & 549 & $\begin{array}{c}525,438, \\
371,320\end{array}$ & 576 \\
\hline DCM & $\begin{array}{c}490,402, \\
330\end{array}$ & 520,457 & $\begin{array}{c}510,360, \\
331\end{array}$ & 584,442 & $\begin{array}{c}512,448, \\
357\end{array}$ & 600 & $\begin{array}{c}524,451, \\
368,322\end{array}$ & 588 \\
\hline EtOAc & $\begin{array}{c}490,391, \\
323\end{array}$ & 517,463 & $\begin{array}{c}504,346, \\
326\end{array}$ & 583,464 & $\begin{array}{c}501,427, \\
345\end{array}$ & 618 & $\begin{array}{c}512,426, \\
347,314\end{array}$ & 572,481 \\
\hline MeCN & $\begin{array}{c}486,396, \\
317\end{array}$ & 520,459 & $\begin{array}{c}502, \\
350,327\end{array}$ & 589,448 & $\begin{array}{c}500,428, \\
346\end{array}$ & 605 & $\begin{array}{c}510,426, \\
353,315\end{array}$ & 583 \\
\hline
\end{tabular}


Table S6. Photophysical properties

\begin{tabular}{|c|c|c|c|c|c|c|c|c|c|}
\hline \multirow[b]{2}{*}{ Molecule } & \multirow{2}{*}{$\begin{array}{c}\begin{array}{c}\text { Absorption } \\
\text { maxima }\end{array} \\
\begin{array}{c}\text { In DCM } \\
(\mathrm{nm})\end{array}\end{array}$} & \multicolumn{2}{|c|}{ Emission maxima } & \multicolumn{2}{|c|}{$\begin{array}{l}\text { Quantum yield } \\
(\Phi)\end{array}$} & \multicolumn{2}{|c|}{$\begin{array}{c}\text { Average lifetime } \\
(\tau)^{(\mathrm{b})}\end{array}$} & \multirow{2}{*}{$\begin{array}{c}\mathrm{k}_{\mathrm{r}} \\
\left(10^{7} \mathrm{~s}^{-1}\right)^{(\mathrm{c})}\end{array}$} & \multirow{2}{*}{$\begin{array}{c}\sum \mathrm{k}_{\mathrm{nr}} \\
\left(10^{9} \mathrm{~s}^{-1}\right)^{(\mathrm{c}}\end{array}$} \\
\hline & & $\begin{array}{c}\text { In DCM } \\
(\mathrm{nm})\end{array}$ & $\begin{array}{c}\text { In solid } \\
\text { state } \\
(\mathrm{nm})\end{array}$ & $\begin{array}{c}\text { In } \\
\text { DCM } \\
(\%)^{(\mathrm{a})} \\
\end{array}$ & $\begin{array}{c}\text { In } \\
\text { solid } \\
\text { state } \\
\end{array}$ & $\begin{array}{c}\text { In DCM } \\
\text { (ns) }\end{array}$ & $\begin{array}{c}\text { In solid } \\
\text { state } \\
\text { (ns) } \\
\end{array}$ & & \\
\hline $4 \mathbf{a}$ & $\begin{array}{c}490,402 \\
330 \\
\end{array}$ & $\begin{array}{l}520 \\
457 \\
\end{array}$ & 503 & 0.30 & 2.85 & 0.6955 & 0.7755 & 0.4313 & 1.433 \\
\hline $4 b$ & $\begin{array}{c}510,360 \\
331\end{array}$ & $\begin{array}{l}584, \\
442 \\
\end{array}$ & 546 & 1.24 & 4.10 & 0.9039 & 1.0019 & 1.3718 & 1.0925 \\
\hline $4 c$ & $\begin{array}{c}513,447 \\
356\end{array}$ & 601 & 595 & 1.82 & - & 1.6482 & - & 1.1042 & 0.5956 \\
\hline $4 d$ & $\begin{array}{l}524,451, \\
368,322\end{array}$ & 588 & 601 & 2.33 & 11.4 & 1.8166 & 1.1529 & 1.2826 & 0.5376 \\
\hline
\end{tabular}

(a) Quantum yield in solution was calculated using Equation $\Phi=\Phi_{R}\left(\mathrm{I} / \mathrm{I}_{R}\right)\left(\mathrm{OD} / \mathrm{OD}_{\mathrm{R}}\right)\left(\mathrm{n}^{2} / \mathrm{n}_{\mathrm{R}}{ }^{2}\right)$, where $\Phi$ is the emission quantum yield, I the integrated emission intensity, OD the optical density, $\mathrm{n}$ the refractive index, and the subscript $\mathrm{R}$ refers to the reference fluorophore of known quantum yield. Fluorescein in $0.1 \mathrm{M} \mathrm{NaOH}(\mathrm{Q} . \mathrm{Y}=0.79)$ was used as the standard

(b) The average lifetime $\tau$ was calculated by using Equation $\tau=\Sigma\left(\alpha_{i} \tau_{i}^{2} / \alpha_{i} \tau_{I}\right)$, where $\alpha_{i}$ is the amplitude of lifetime component and $\tau_{\mathrm{I}}$ is the respective lifetime value.

(c) Radiative $\left(\mathrm{k}_{\mathrm{r}}\right)$ and non-radiative $\left(\Sigma \mathrm{k}_{\mathrm{nr}}\right)$ rate constants estimated using the approximation that the emissive state is formed with unitary efficiency and thus $\mathrm{k}_{\mathrm{r}}=\Phi / \tau$ and $\Sigma \mathrm{k}_{\mathrm{nr}}=(1-\Phi) / \tau$. 


\section{TD-DFT Calculations}

The nature of electronic transitions in these molecules was assessed using TD-DFT method, taking $\mathbf{4 d}$ as a representative example. Initially, the geometry was optimized using B3LYP functional method and 631G(d) basis set in Gaussian 16. The optimized geometry was then subjected to the TD-DFT analysis using B3LYP/6-31G(d) in two solvents, namely dichloromethane (DCM) and acetonitrile (MeCN). The structures of molecular orbitals were analysed using Chemcraft software. Energies of these orbitals, their types and shapes (from the experiment done using dichloromethane as the solvent) are shown in Figure S6. It is evident that the occupied molecular orbitals HOMO, HOMO-1, HOMO-3, HOMO-4 are mainly located on the C-2 and C-3 diaryl units whereas HOMO-2 is delocalized over one of the aryl rings (C-3) and the azatricyclic unit. At the same time, the unoccupied orbitals LUMO, LUMO+1 are located mainly on the azatricyclic unit. Since the occupied orbitals are primarily located on electron-rich aryl fragments and the unoccupied orbitals are on the aza-tricyclic unit, the electronic transitions involving these MOs can be viewed as having intramolecular charge transfer characteristics.

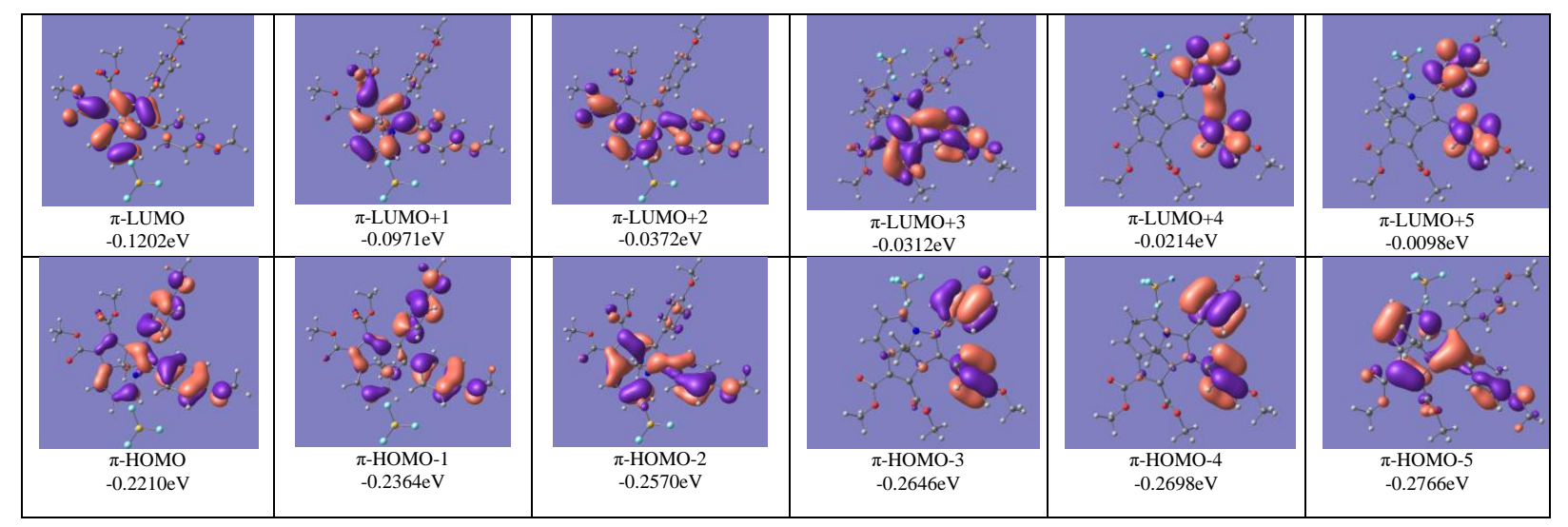

Figure S6. MOs of $\mathbf{4 d}$ (DCM was used as the medium)

TD-DFT calculations were used to simulate the UV spectra and to determine the MOs involved in each of the transitions. These calculations were performed in MeCN and DCM using B3LYP functional method. The experimentally determined absorption maxima and theoretically calculated UV spectral data in DCM and MeCN solvents are given in Table S7. The theoretically calculated absorption at $533 \mathrm{~nm}$ with an oscillator strength of 0.2325 arises from an intramolecular charge transfer from HOMO to the LUMO. The absorptions at $386 \mathrm{~nm}$ (oscillator strength $=0.2632$ ) and $376 \mathrm{~nm}$ (oscillator strength $=$ 0.2953) involve HOMO-2 to LUMO and HOMO-1 to LUMO+1 transition, respectively. Based on the contributions of aryl- and aza-tricyclic units to these MOs, these transitions can be considered as having 
intramolecular charge transfer features. The calculated absorption data in DCM and MeCN are comparable in terms of absorption wavelength, corresponding oscillator strength and the MOs involved.

Table S7. Comparison of experimental and calculated UV spectral data.

\begin{tabular}{|c|c|c|c|c|c|c|}
\hline \multirow[b]{2}{*}{ Entry } & \multirow[b]{2}{*}{ Solvent } & \multirow{2}{*}{$\begin{aligned} \text { Experimental }^{\mathrm{a}} \\
\lambda_{\max }{ }^{\exp }(\mathrm{nm})\end{aligned}$} & \multicolumn{2}{|c|}{ Calculated (TD-DFT) ${ }^{\mathrm{b}}$} & \multirow[b]{2}{*}{ Transition $^{c}$} & \multirow[b]{2}{*}{ Assignment } \\
\hline & & & $\lambda_{\max }{ }^{\mathrm{cal}}(\mathrm{nm})$ & Osc.strength & & \\
\hline 1 & DCM & 524,451 & 533 & 0.2325 & $\mathrm{H} \rightarrow \mathrm{L}$ & $\pi \rightarrow \pi *$ \\
\hline 2 & & 368,322 & 386 & 0.2632 & $\mathrm{H}-2 \rightarrow \mathrm{L}$ & $\pi \rightarrow \pi *$ \\
\hline 3 & & & 376 & 0.2953 & $\mathrm{H}-1 \longrightarrow \mathrm{L}+1$ & $\pi \rightarrow \pi *$ \\
\hline 4 & & & 338 & 0.0776 & $\mathrm{H}-5 \rightarrow \mathrm{L}$ & $\pi \longrightarrow \pi *$ \\
\hline 5 & & & 314 & 0.0831 & $\mathrm{H}-3 \rightarrow \mathrm{L}+1$ & $\pi \rightarrow \pi *$ \\
\hline 6 & $\mathrm{MeCN}$ & 510,426 & 532 & 0.2218 & $\mathrm{H} \rightarrow \mathrm{L}$ & $\pi \rightarrow \pi *$ \\
\hline 7 & & 353,315 & 384 & 0.2374 & $\mathrm{H}-2 \rightarrow \mathrm{L}$ & $\pi \rightarrow \pi *$ \\
\hline 8 & & & 375 & 0.3023 & $\mathrm{H}-1 \rightarrow \mathrm{L}+1$ & $\pi \rightarrow \pi *$ \\
\hline 9 & & & 338 & 0.0878 & $\mathrm{H}-5 \longrightarrow \mathrm{L}$ & $\pi \rightarrow \pi *$ \\
\hline 10 & & & 313 & 0.0695 & $\mathrm{H}-3 \longrightarrow \mathrm{L}+1$ & $\pi \rightarrow \pi *$ \\
\hline
\end{tabular}

${ }^{a}$ Peak maxima were determined using a second derivative based method. ${ }^{b}$ TD-B3LYP/6-31G(d). ${ }^{c} \mathrm{H}-$ highest occupied MO (HOMO), L-lowest unoccupied MO (LUMO). 


\section{Copy of NMR spectra of compounds}

$\mathrm{JK}-01-3 \mathrm{a}$

ittm_PROTON-5to15 CDCl3/opt/topspin nmr 12

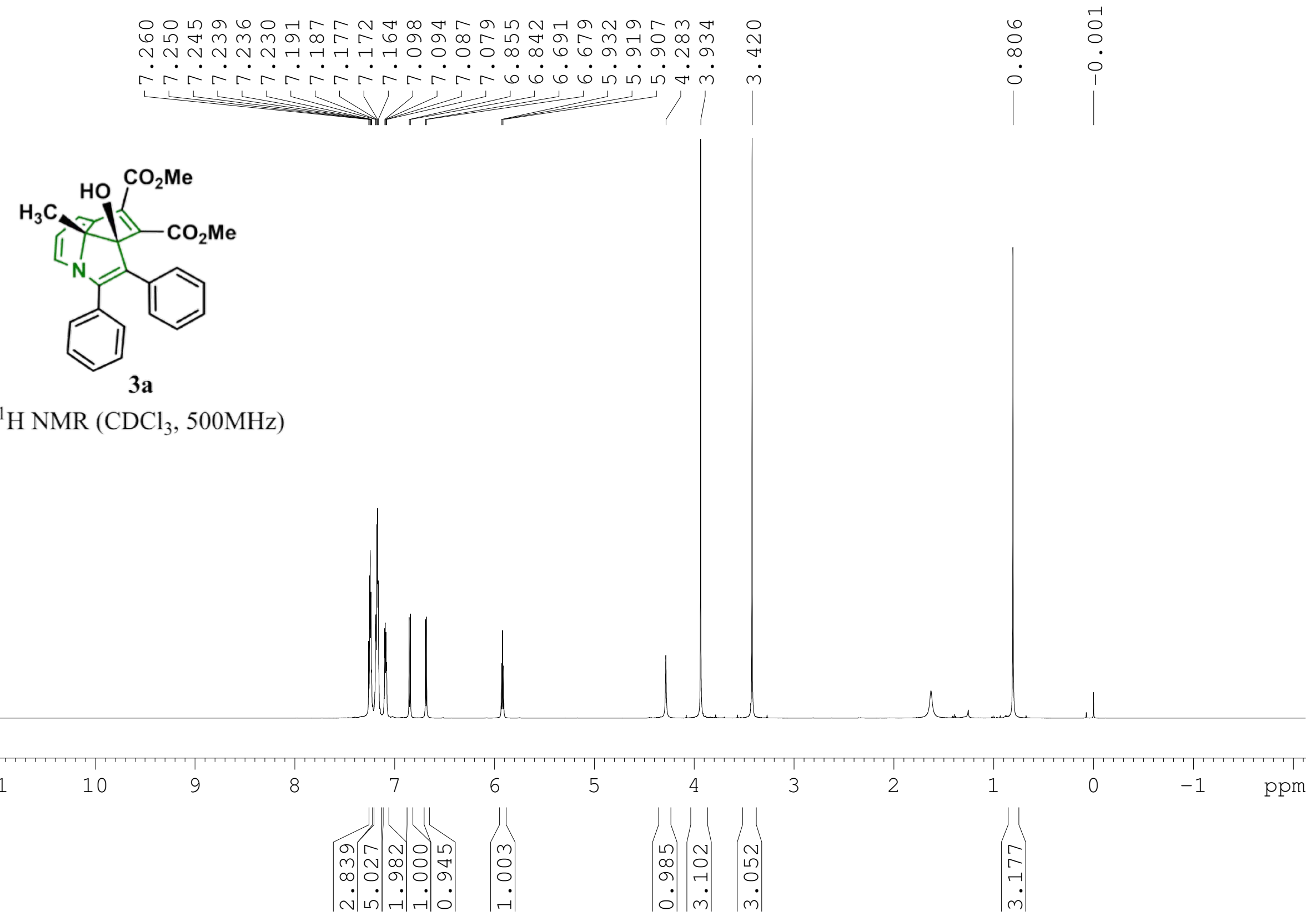

Figure S7: 1H NMR (500 MHz) spectrum of 3a 

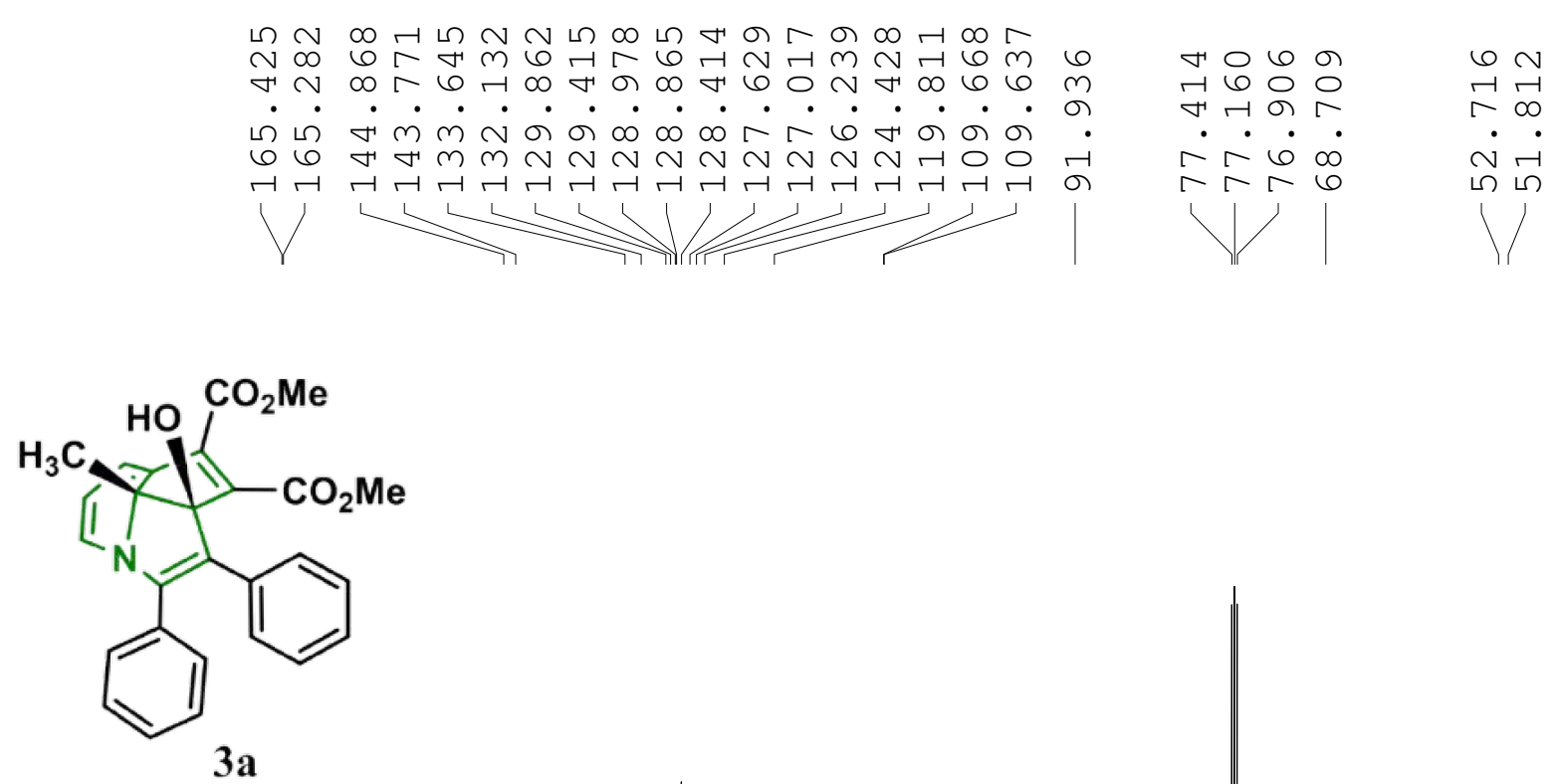

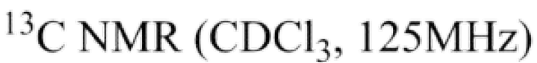



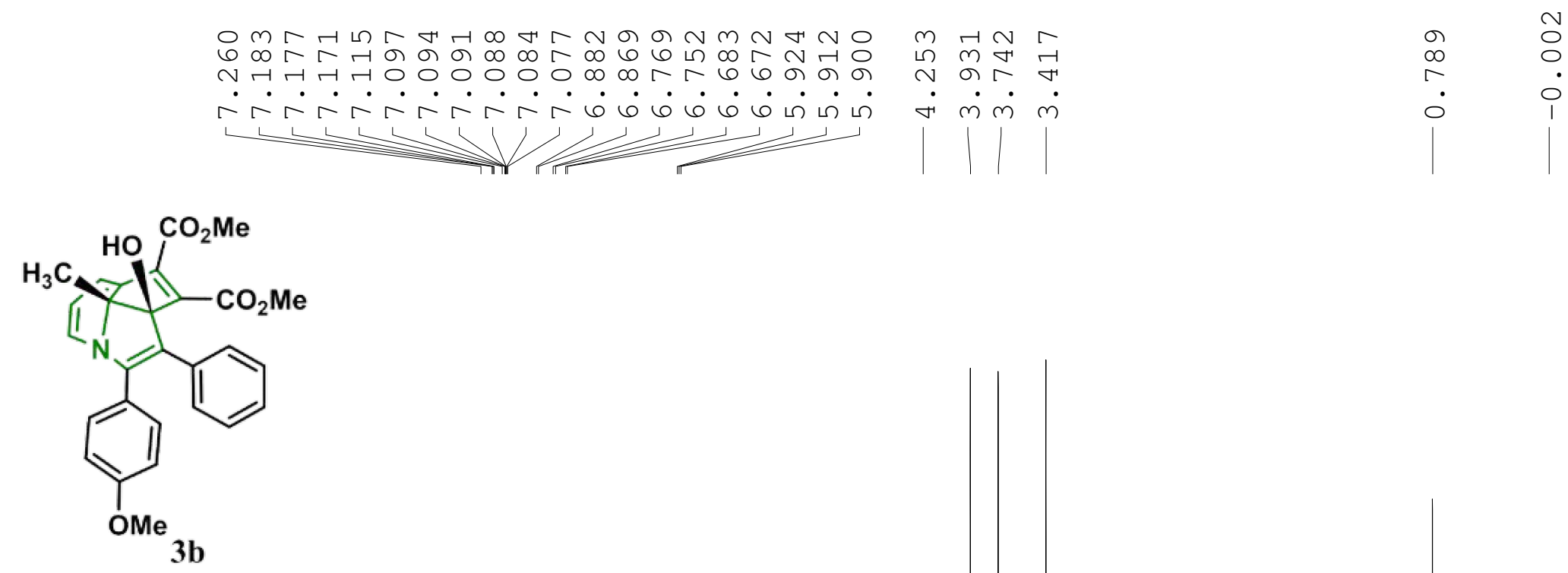

${ }^{1} \mathrm{H} \mathrm{NMR}\left(\mathrm{CDCl}_{3}, 500 \mathrm{MHz}\right)$

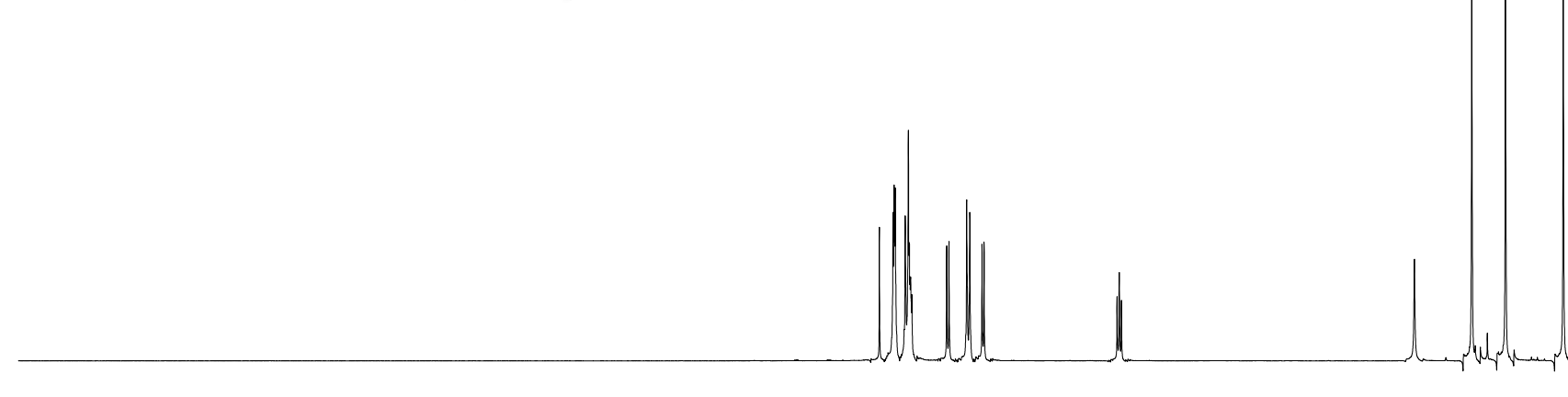

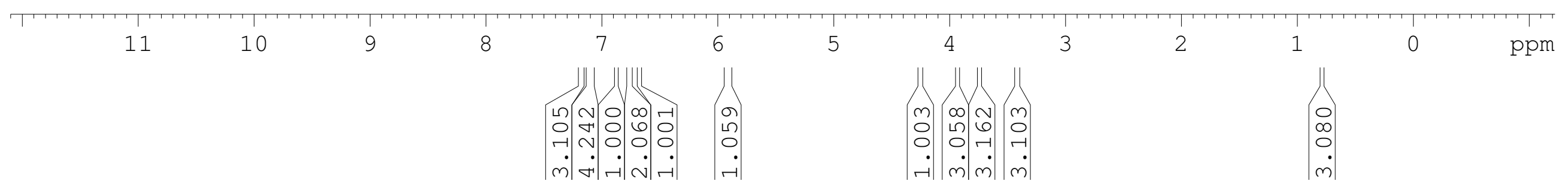

Figure S9: 1H NMR (500 MHz) spectrum of 3b 

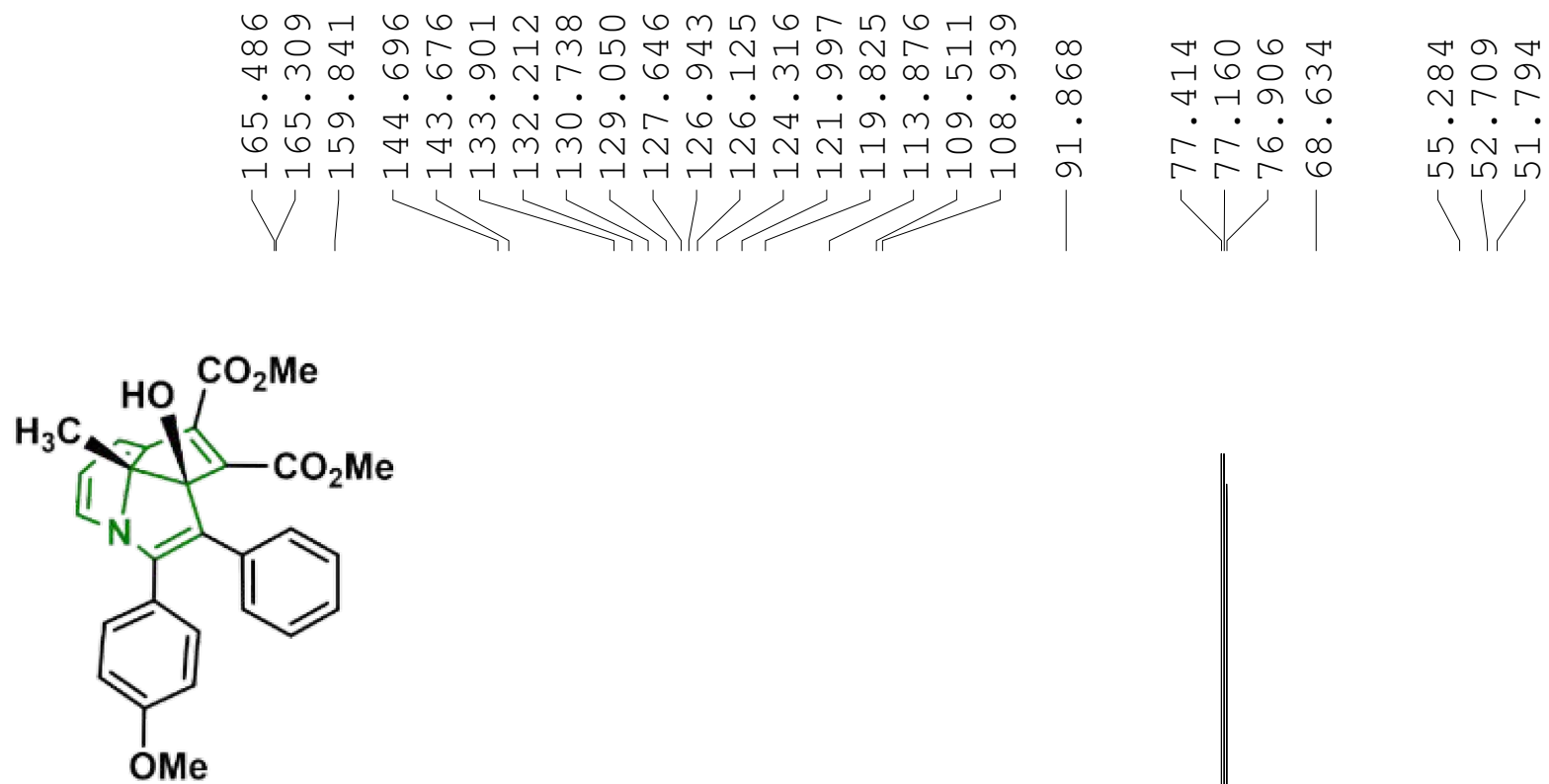

3b

${ }^{13} \mathrm{C} \mathrm{NMR}\left(\mathrm{CDCl}_{3}, 125 \mathrm{MHz}\right)$
N

$\stackrel{0}{6}$

$\dot{m}$ 


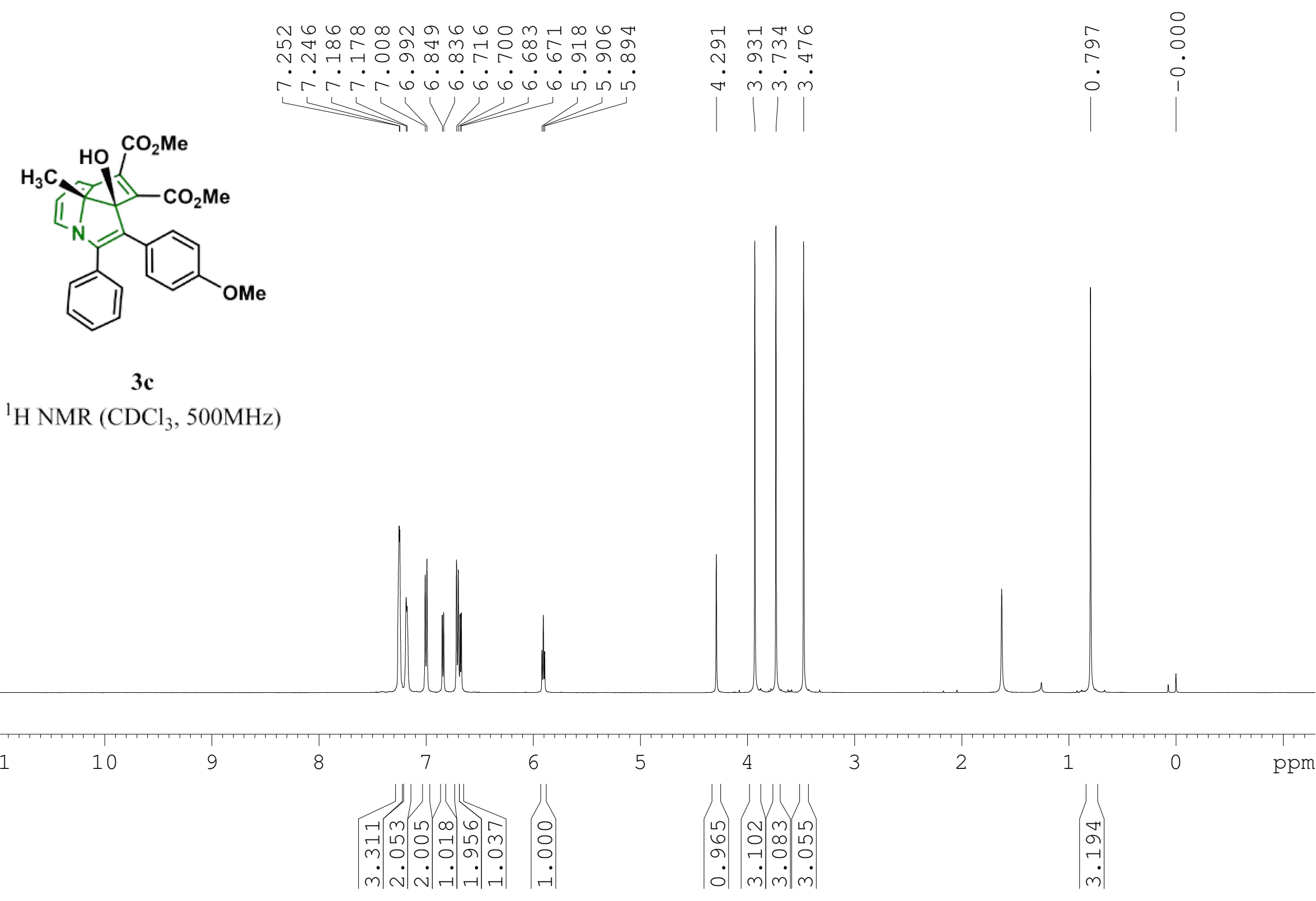



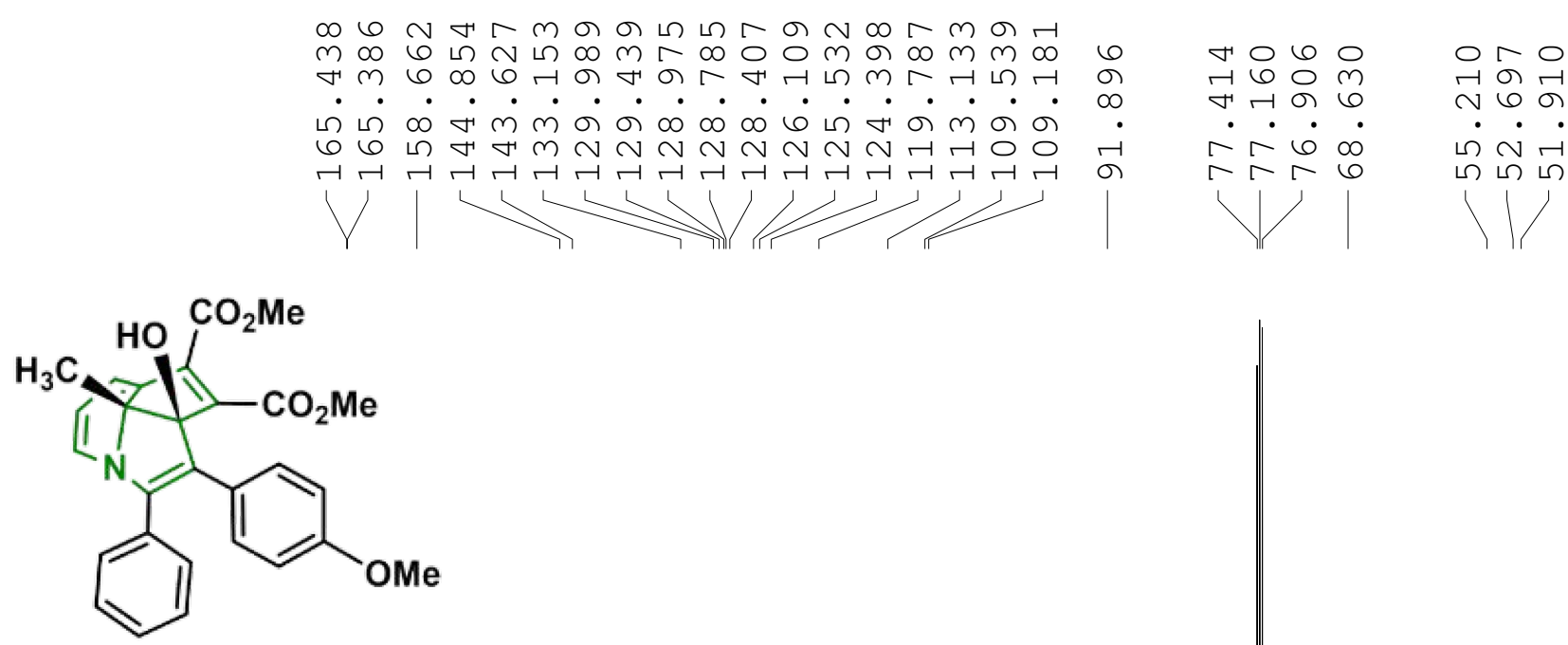

$3 \mathrm{c}$

${ }^{13} \mathrm{C} \mathrm{NMR}\left(\mathrm{CDCl}_{3}, 125 \mathrm{MHz}\right)$ 


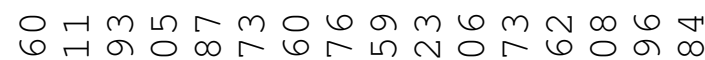
N

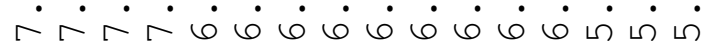

の느의

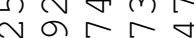

$\dot{\sigma} \dot{m} \dot{m} \dot{m} \dot{m}$

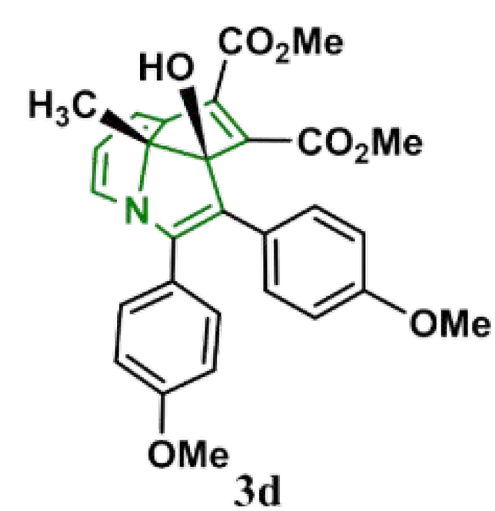

${ }^{1} \mathrm{H} \mathrm{NMR}\left(\mathrm{CDCl}_{3}, 500 \mathrm{MHz}\right)$

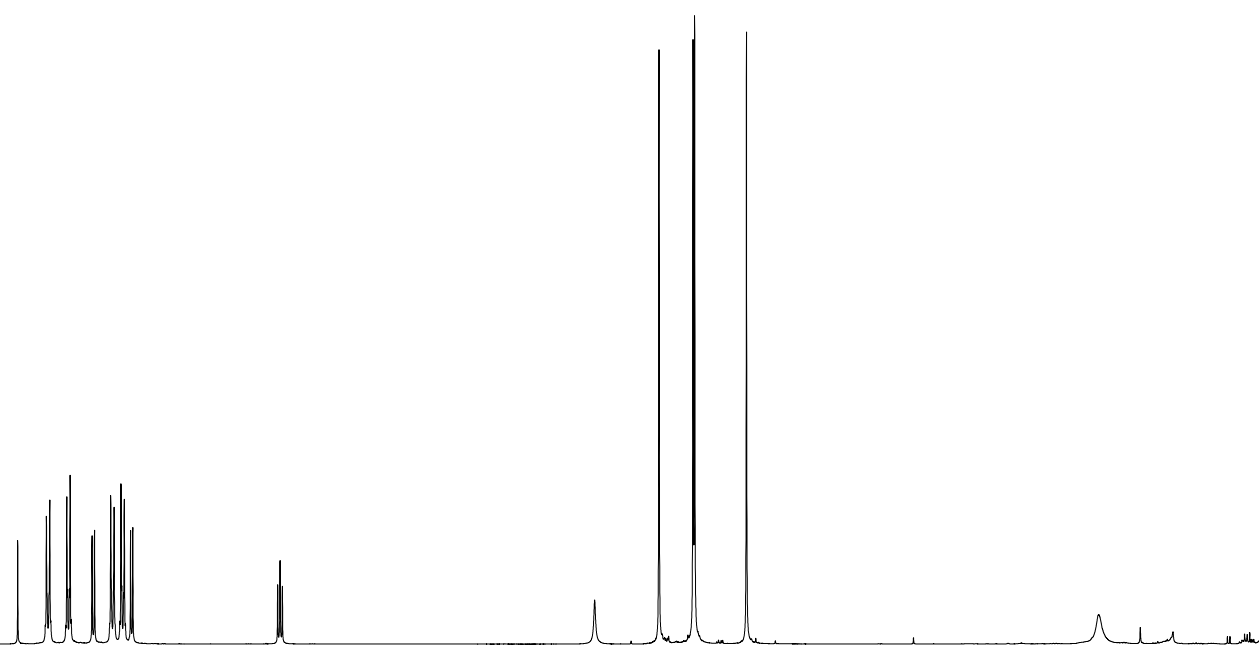

9

8

7

5

4

3

2

1

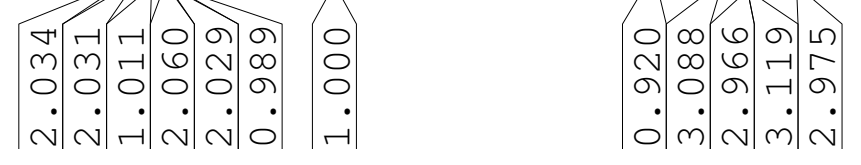



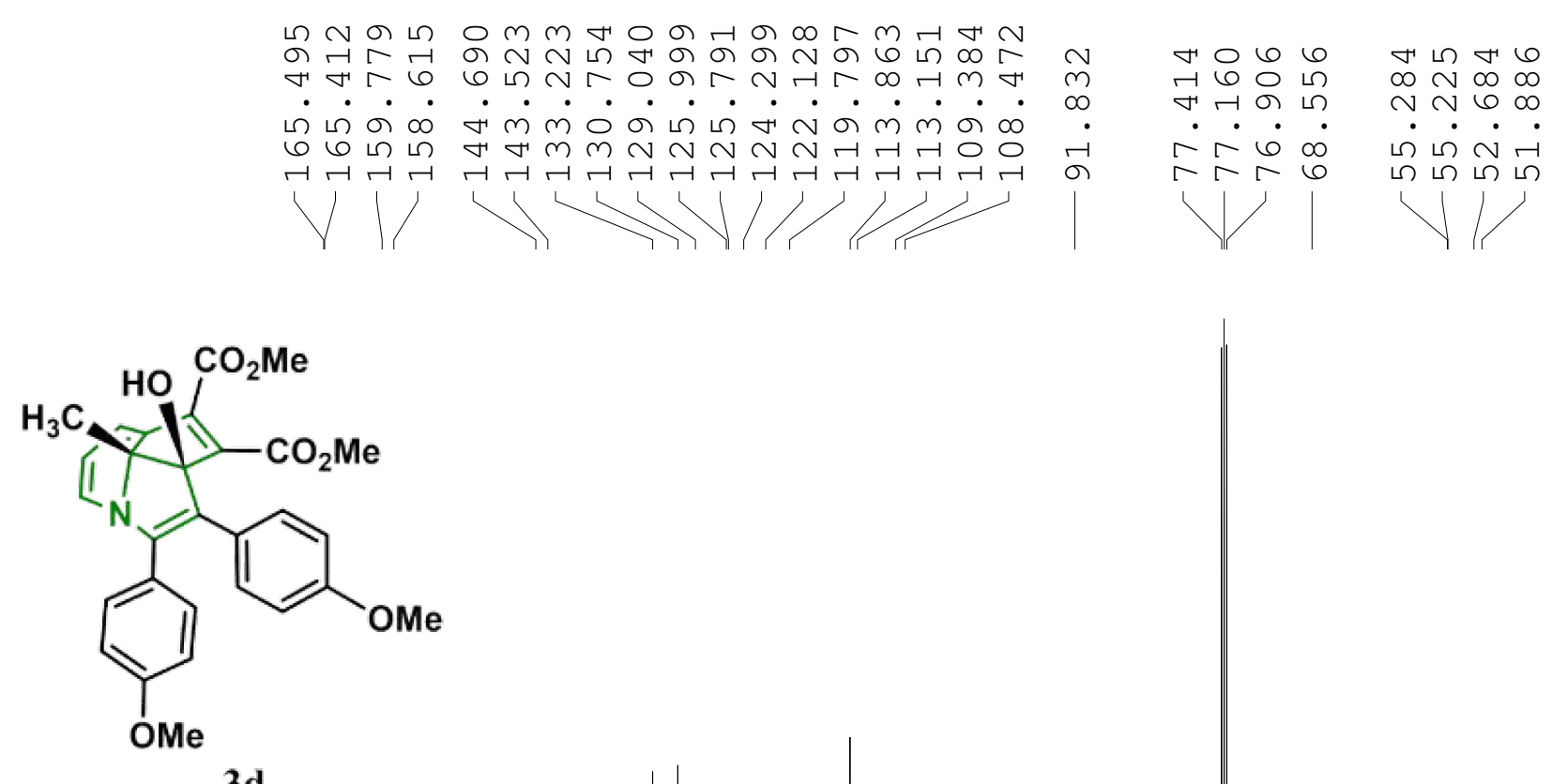

${ }^{13} \mathrm{C} \mathrm{NMR}\left(\mathrm{CDCl}_{3}, 125 \mathrm{MHz}\right)$ 

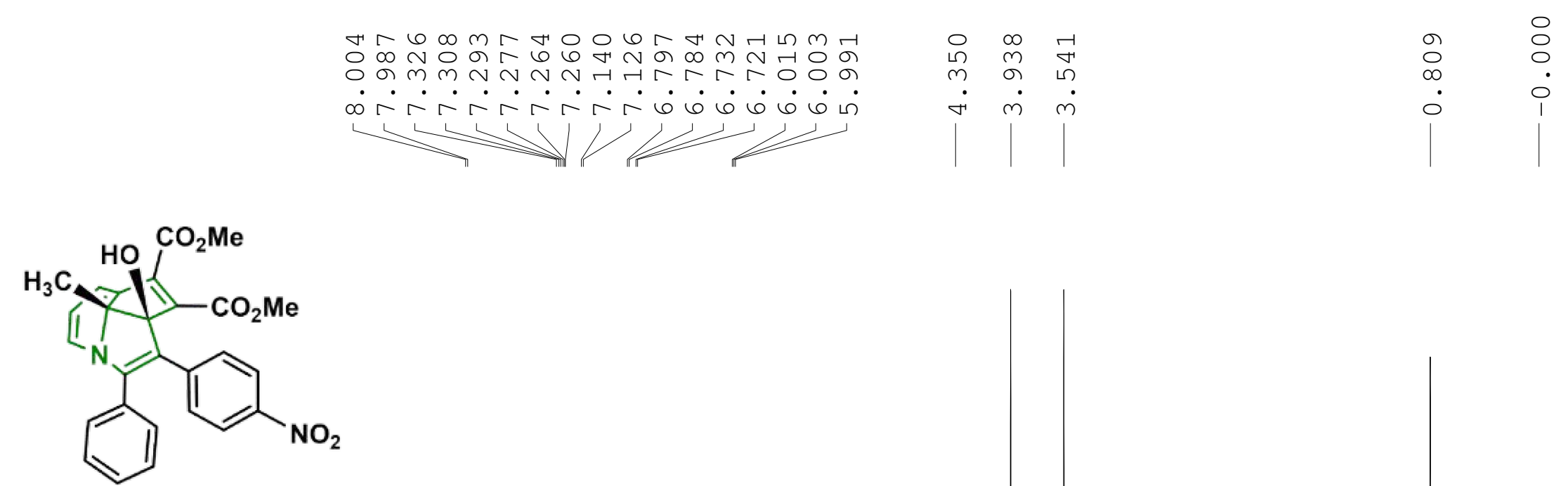

$3 e$

${ }^{1} \mathrm{H}$ NMR $\left(\mathrm{CDCl}_{3}, 500 \mathrm{MHz}\right)$

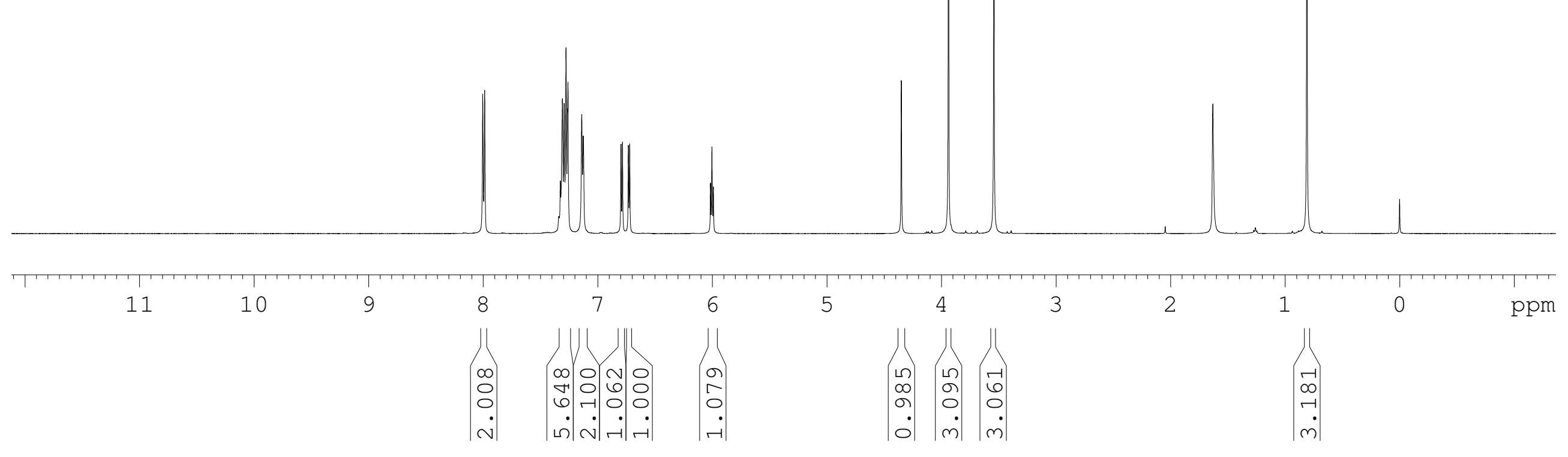

Figure S15: 1H NMR (500 MHz) spectrum of $3 \mathrm{e}$ 

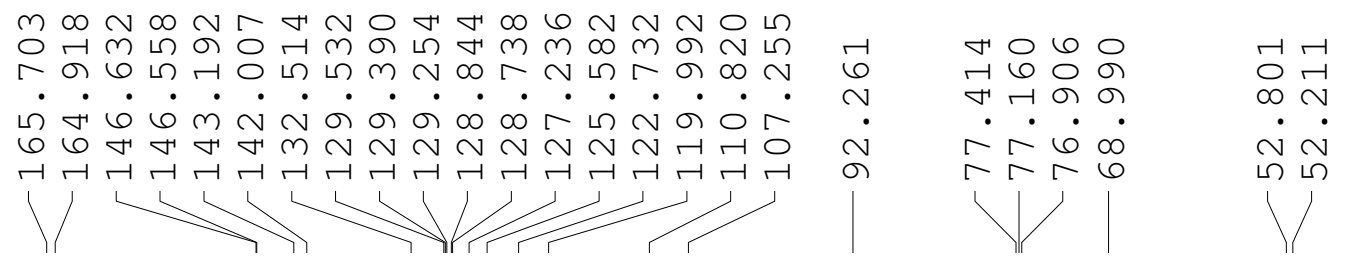

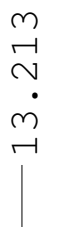

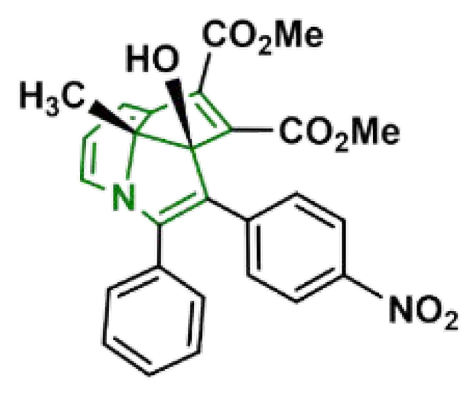

$3 e$

${ }^{13} \mathrm{C} \mathrm{NMR}\left(\mathrm{CDCl}_{3}, 125 \mathrm{MHz}\right)$ 


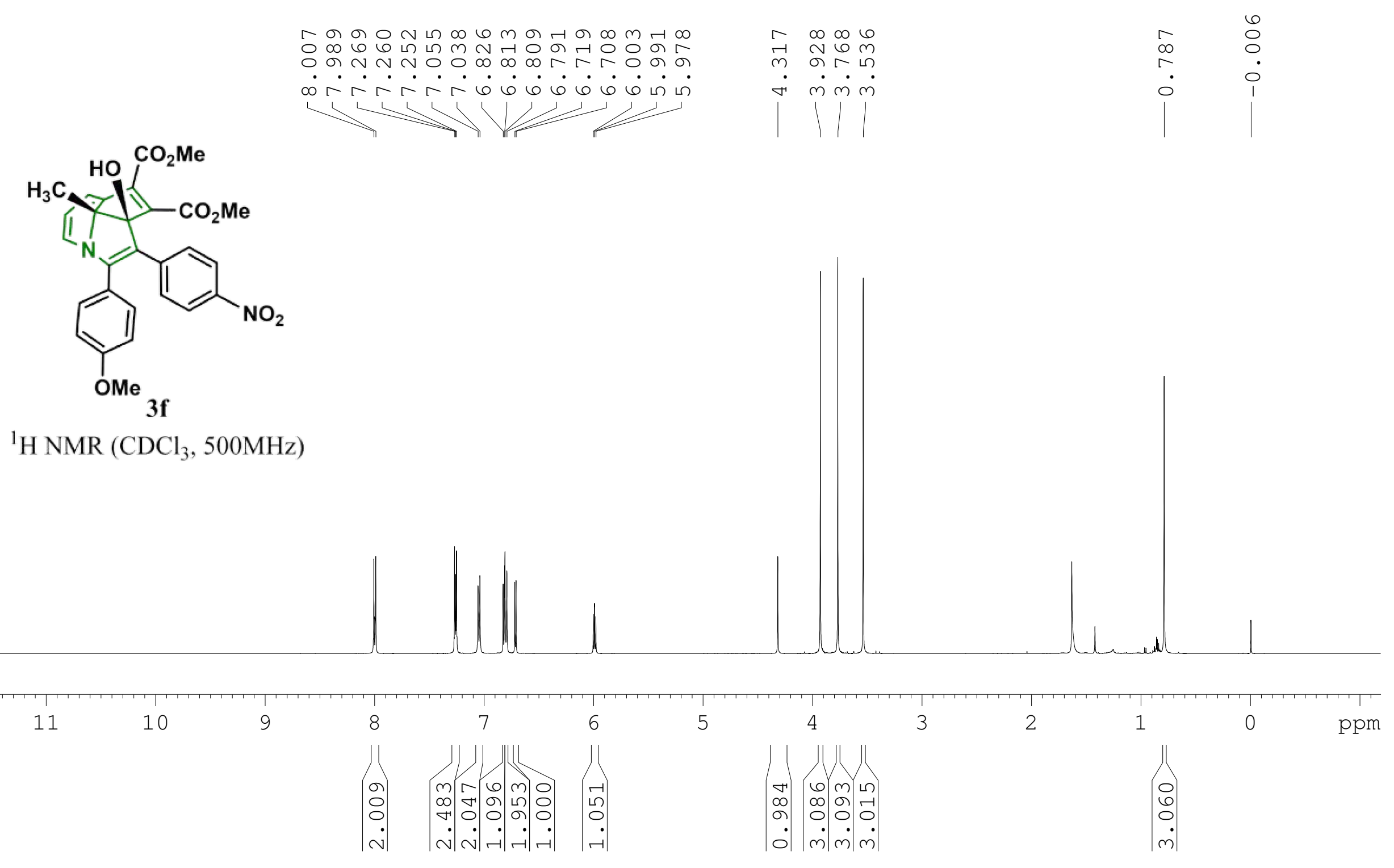

Figure S17: $1 \mathrm{H}$ NMR $(500 \mathrm{MHz})$ spectrum of $3 \mathrm{f}$ 

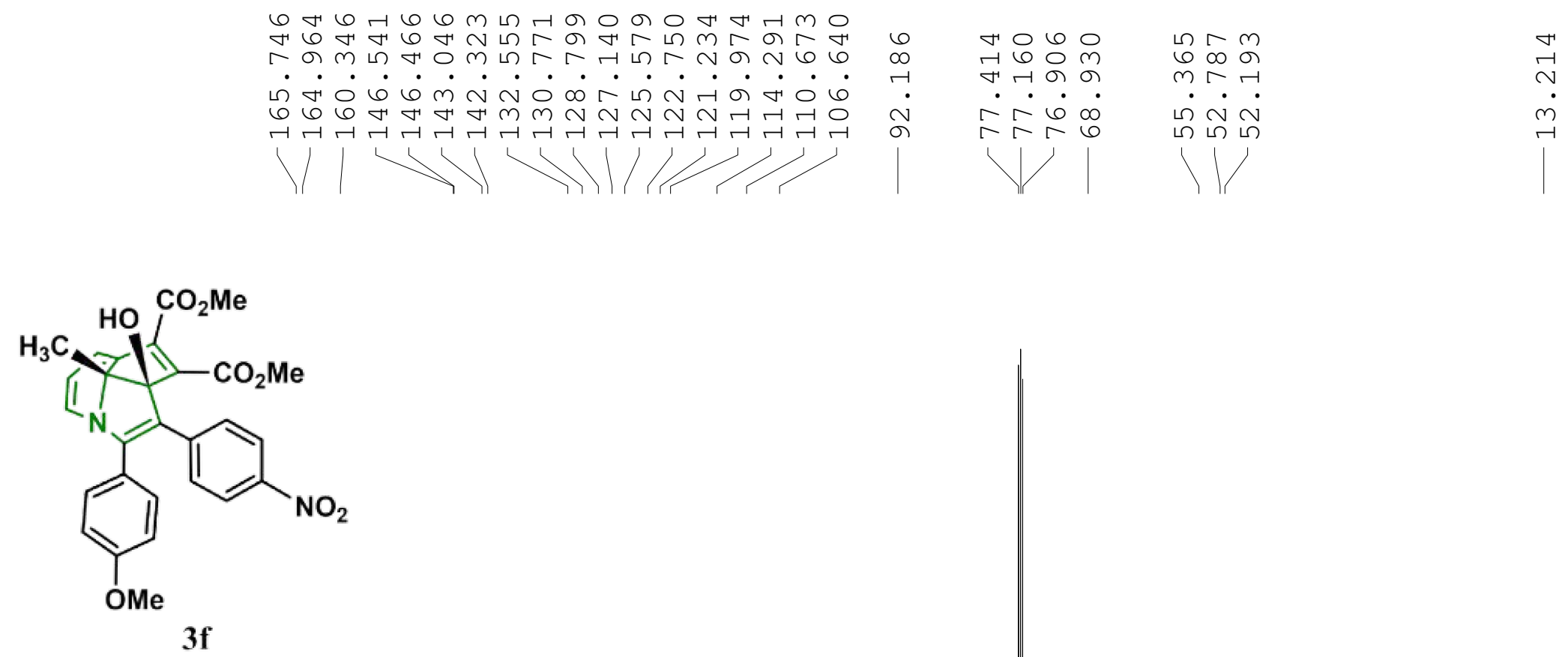

${ }^{13} \mathrm{C} \mathrm{NMR}\left(\mathrm{CDCl}_{3}, 125 \mathrm{MHz}\right)$

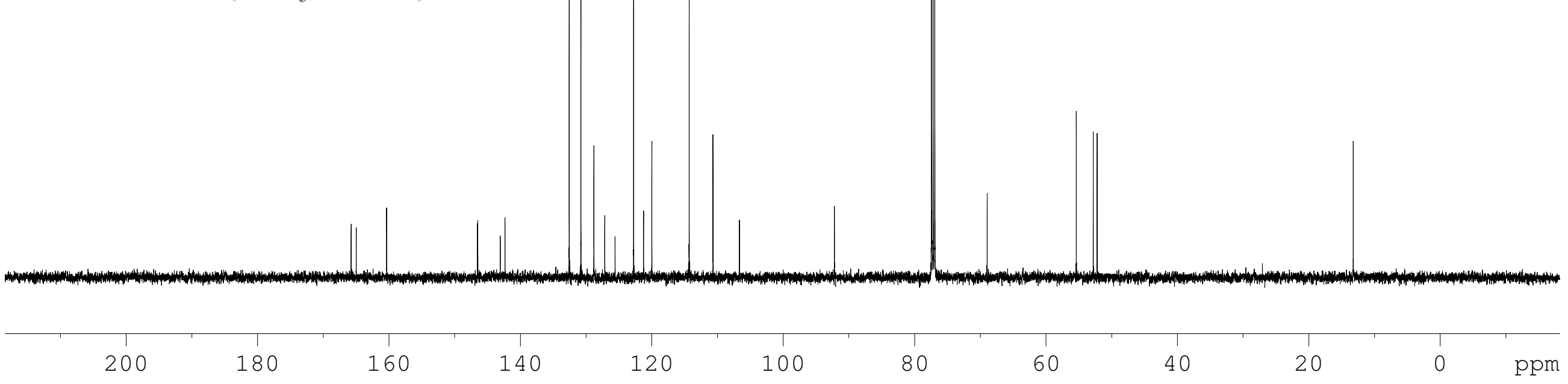

Figure S18: $13 \mathrm{C}$ NMR (125 MHz) spectrum of $3 f$ 

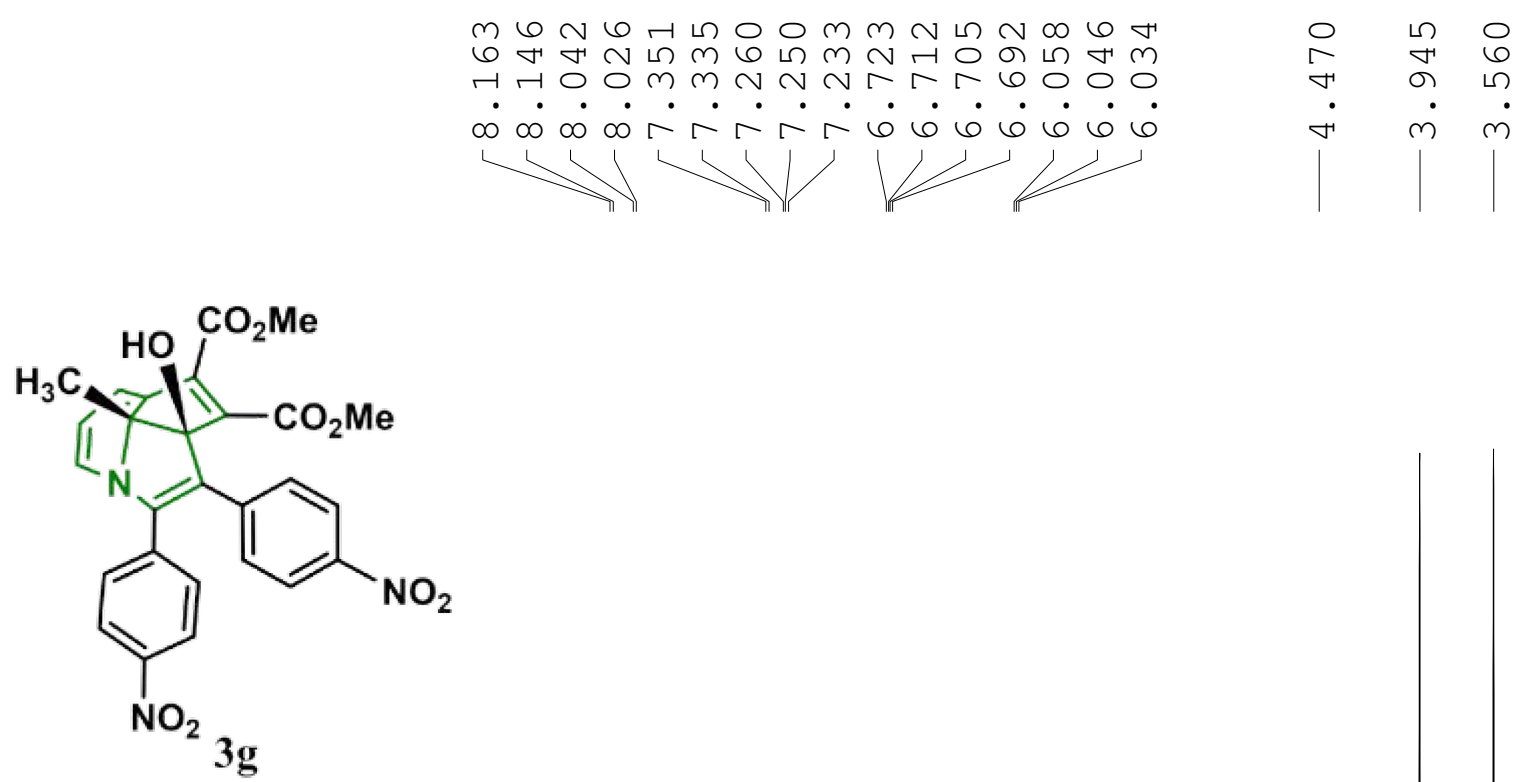

${ }^{1} \mathrm{H}$ NMR $\left(\mathrm{CDCl}_{3}, 500 \mathrm{MHz}\right)$

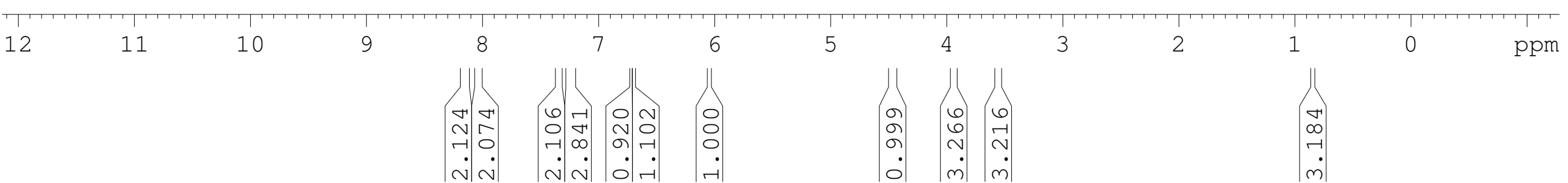

Figure S19: 1H NMR (500 MHz) spectrum of 3g 


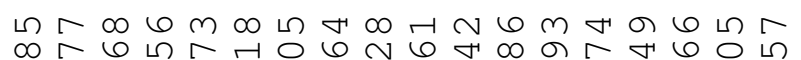

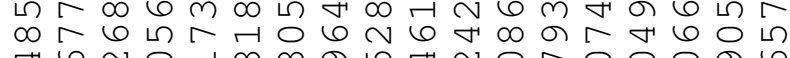
U.

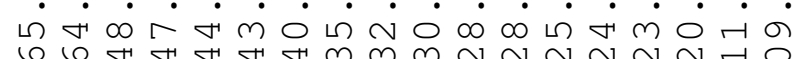

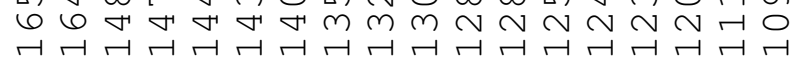

O)

กำ

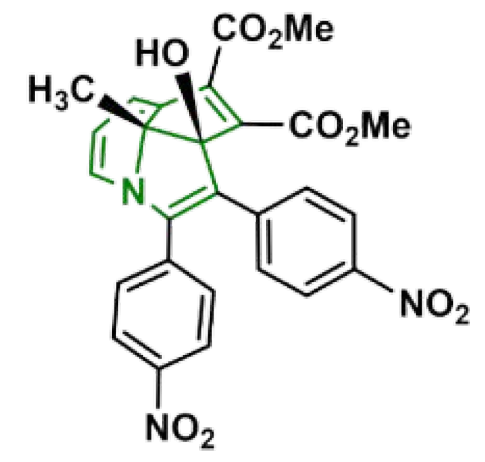

$3 \mathrm{~g}$

${ }^{13} \mathrm{C} \mathrm{NMR}\left(\mathrm{CDCl}_{3}, 125 \mathrm{MHz}\right)$ 
の

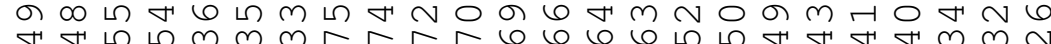

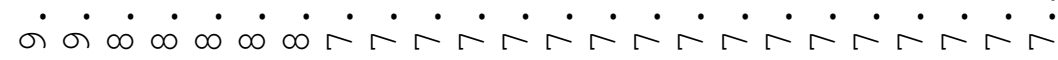

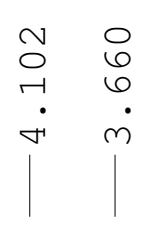

$\stackrel{0}{\infty} \infty$

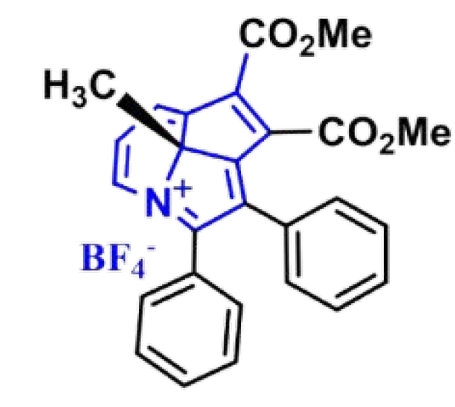

$4 a$

${ }^{1} \mathrm{H} \mathrm{NMR}\left(\mathrm{CDCl}_{3}, 500 \mathrm{MHz}\right)$

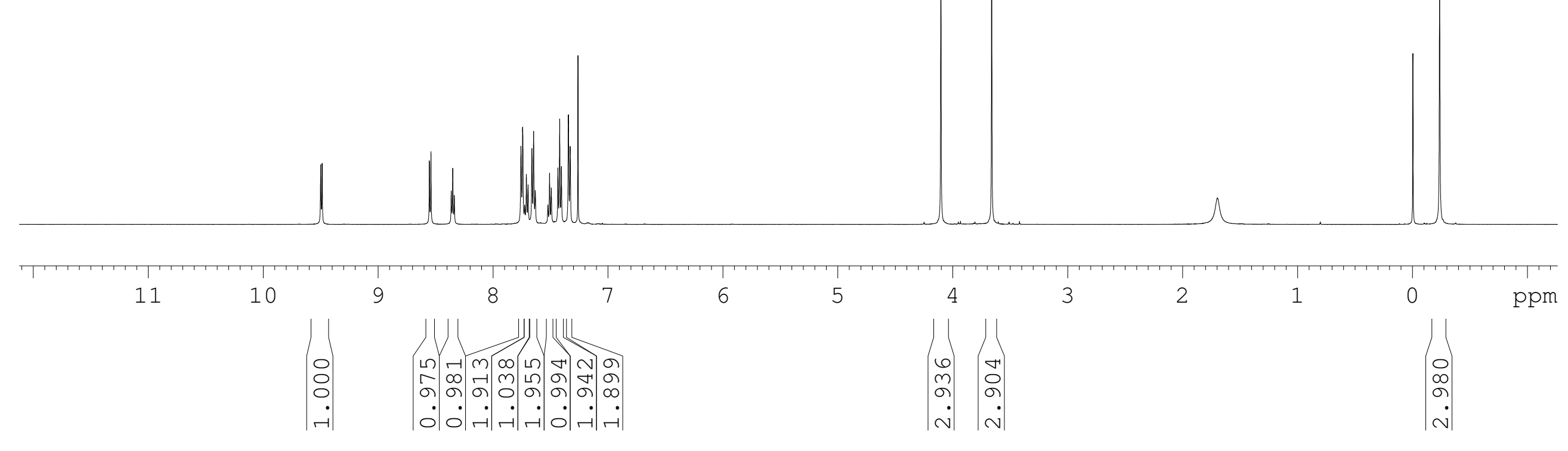




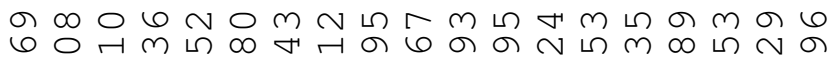

mनrLm

म

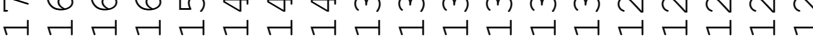

$\stackrel{\sim}{\sim}$

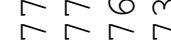

$\infty m$

$\dot{n} \dot{n}$

เก

กे
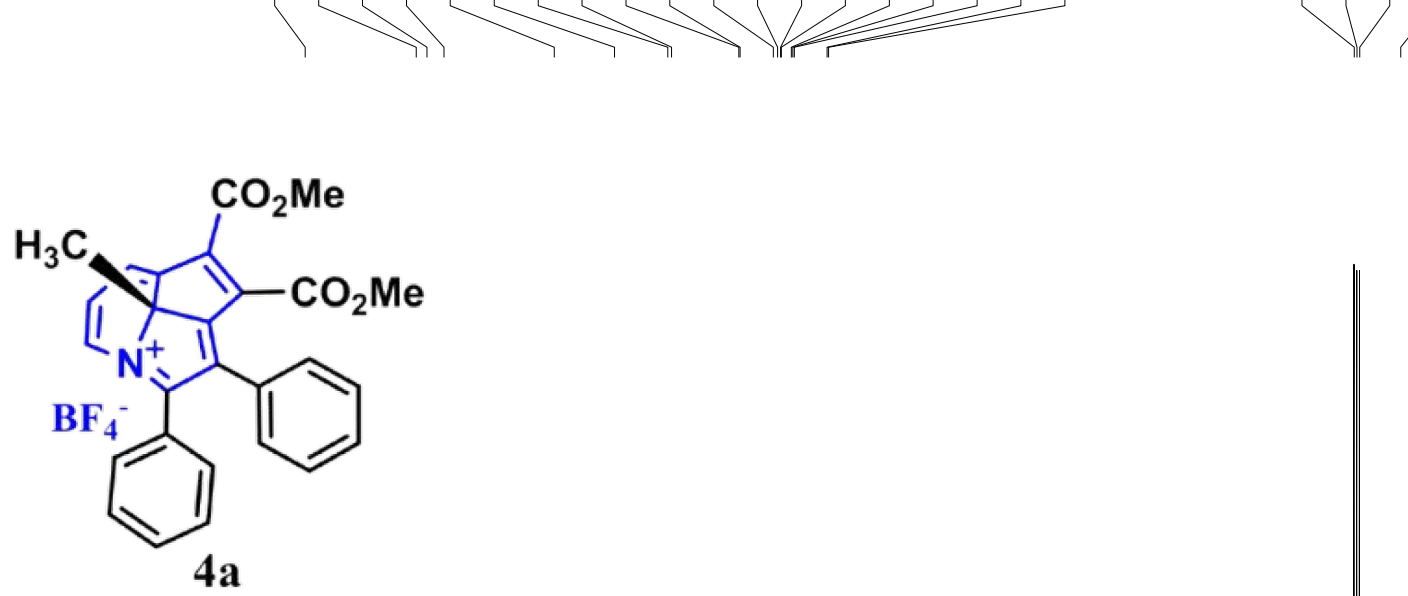

${ }^{13} \mathrm{C} \mathrm{NMR}\left(\mathrm{CDCl}_{3}, 125 \mathrm{MHz}\right)$

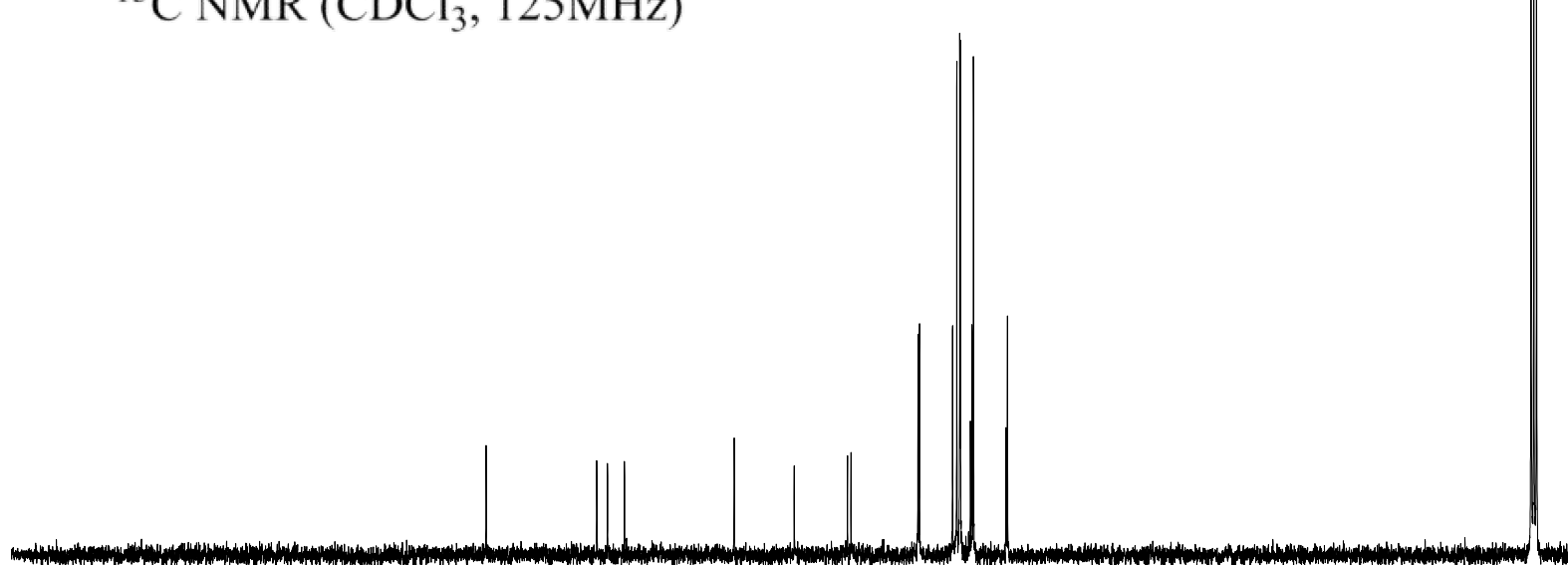

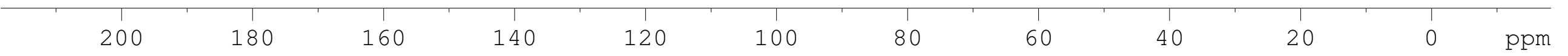




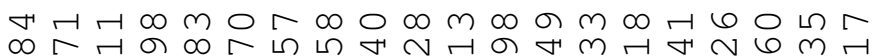

का

बंள்

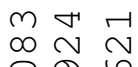

$\dot{\nabla} \dot{m} \dot{m}$

T

$\begin{array}{lll}0 & 0 \\ 0 & \stackrel{4}{r}\end{array}$

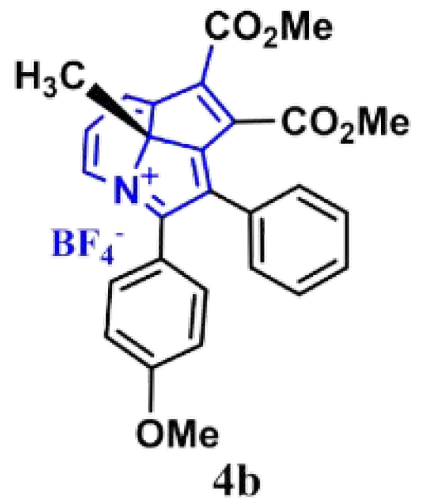

${ }^{1} \mathrm{H} \mathrm{NMR}\left(\mathrm{CDCl}_{3}, 500 \mathrm{MHz}\right)$
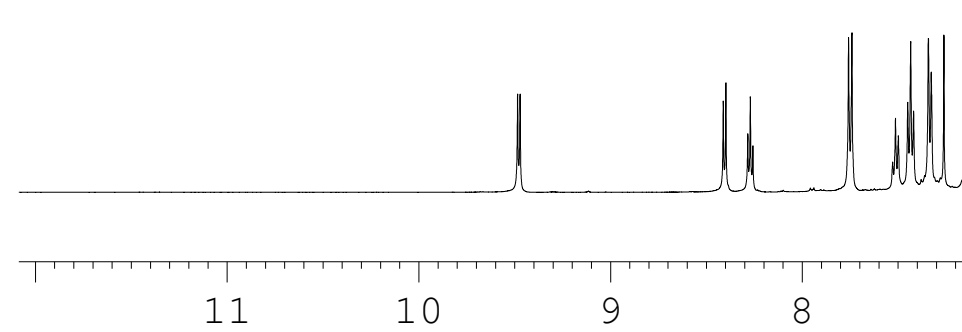

10

9

8

6

5

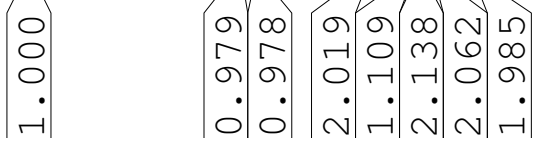

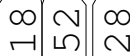

ㅇำ.

$\dot{m} \dot{m} \dot{\sim}$ 


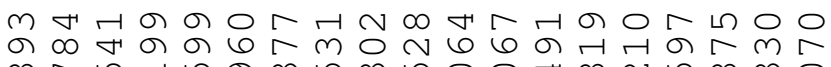

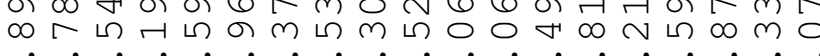

$m$ Fm

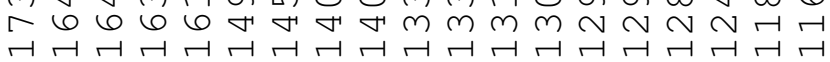

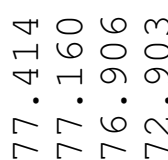

$\infty$ ॠ न

$00 \sim$

ริก

เ

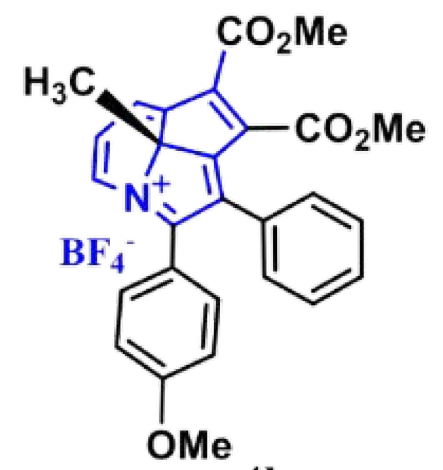

$4 b$

${ }^{13} \mathrm{C} \mathrm{NMR}\left(\mathrm{CDCl}_{3}, 125 \mathrm{MHz}\right)$

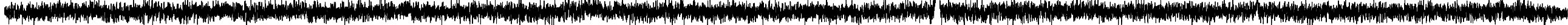



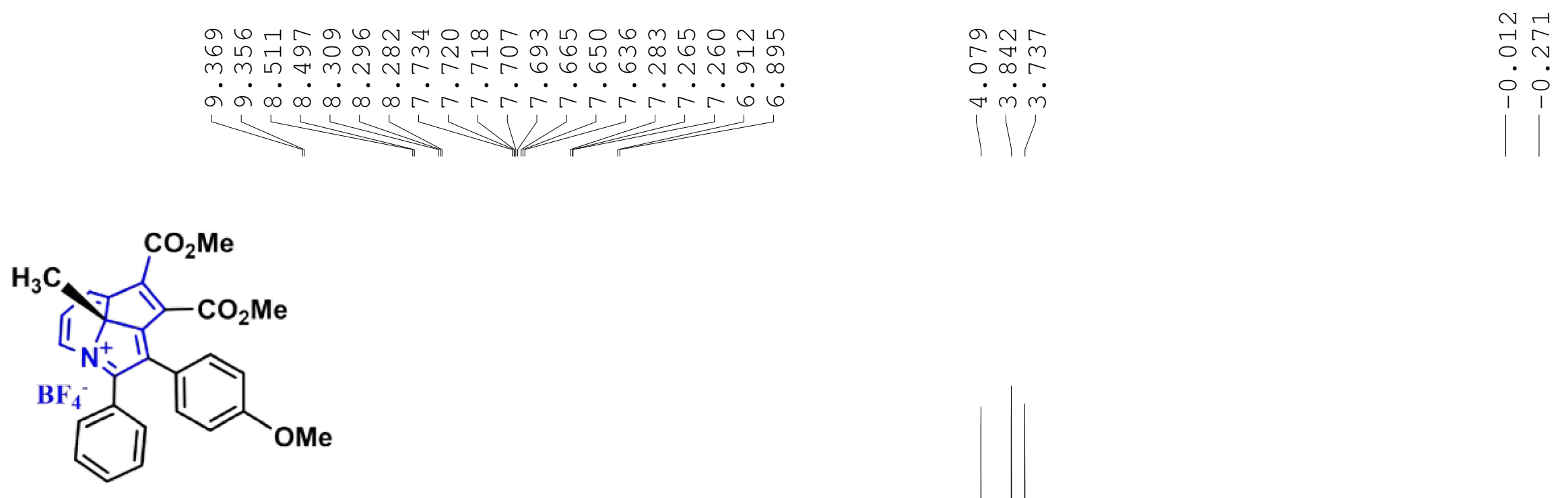

$4 c$

${ }^{1} \mathrm{H} \mathrm{NMR}\left(\mathrm{CDCl}_{3}, 500 \mathrm{MHz}\right)$

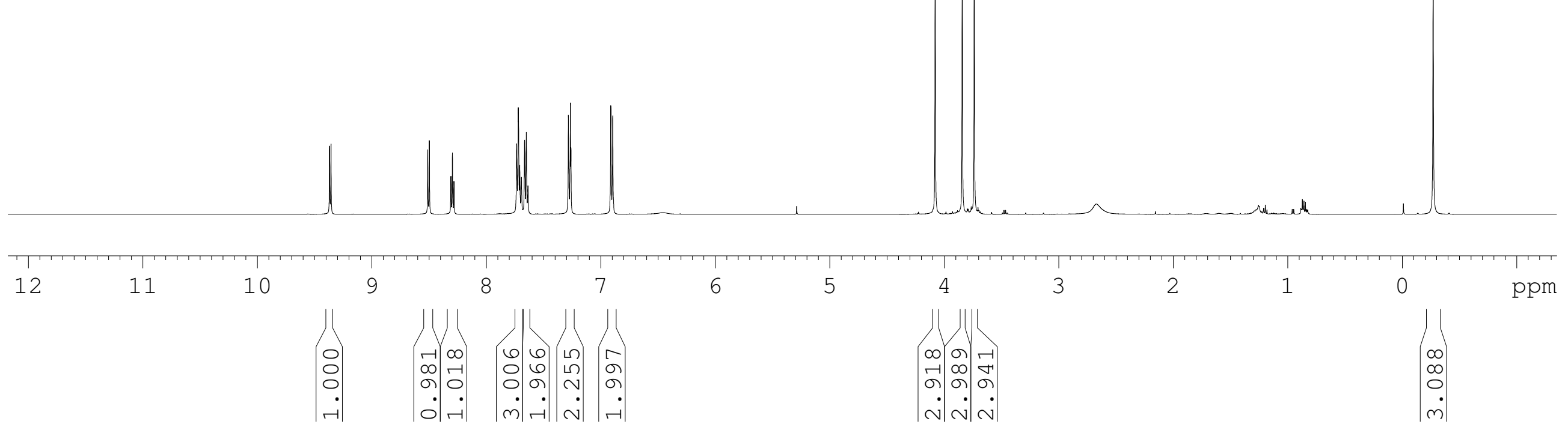



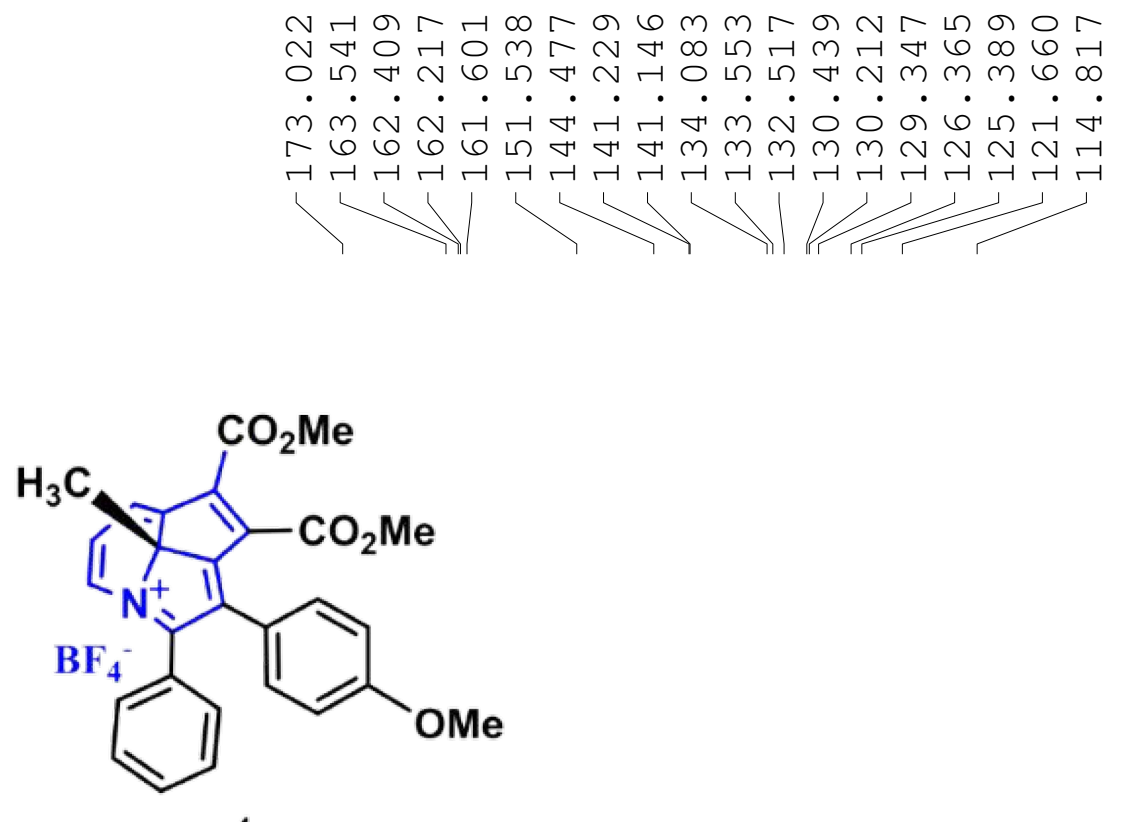

$4 \mathrm{c}$

${ }^{13} \mathrm{C}$ NMR $\left(\mathrm{CDCl}_{3}, 125 \mathrm{MHz}\right)$

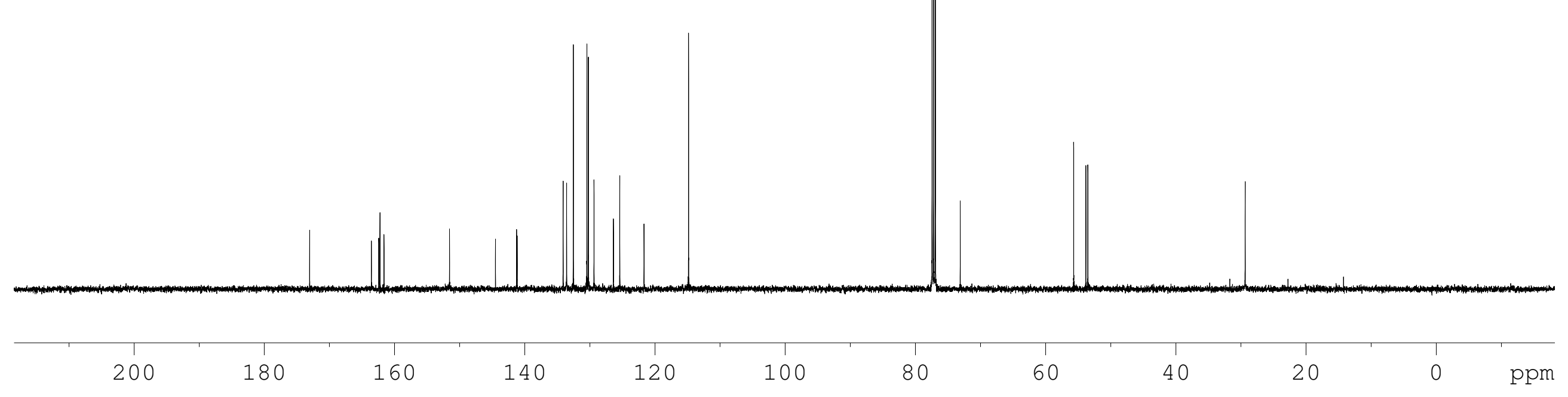




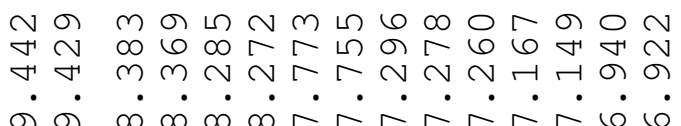

$$
\begin{aligned}
& \text { の } \\
& \dot{\nabla} \dot{m} \dot{m}
\end{aligned}
$$$$
\text { のं் }
$$

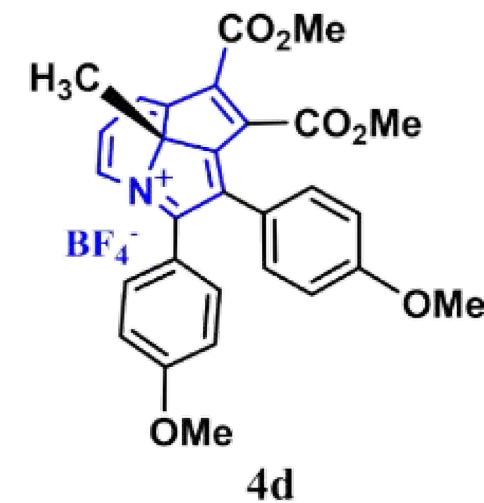

${ }^{1} \mathrm{H}$ NMR $\left(\mathrm{CDCl}_{3}, 500 \mathrm{MHz}\right)$ 

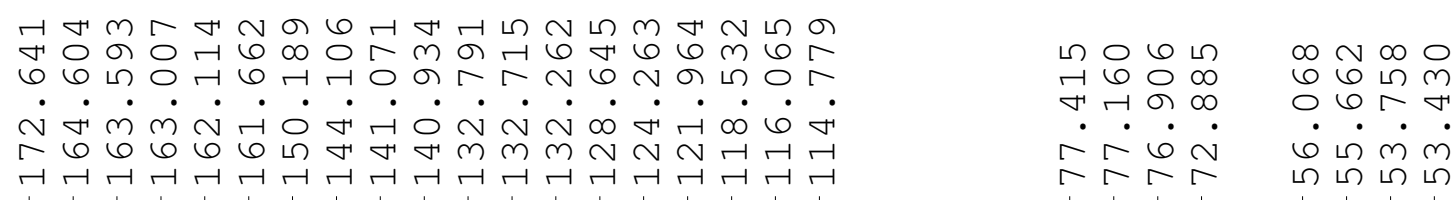

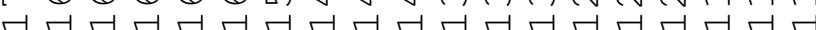

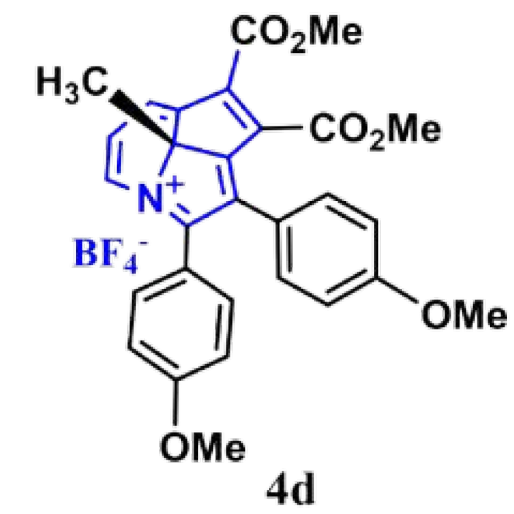

${ }^{13} \mathrm{C} \mathrm{NMR}\left(\mathrm{CDCl}_{3}, 125 \mathrm{MHz}\right)$

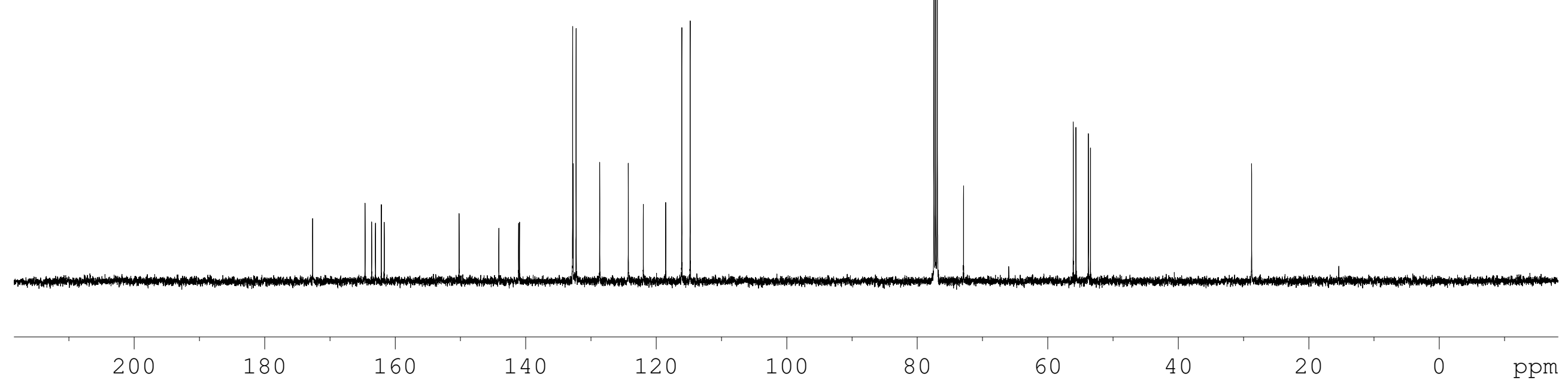

Figure S28: 13C NMR (125 MHz) spectrum of 4d 

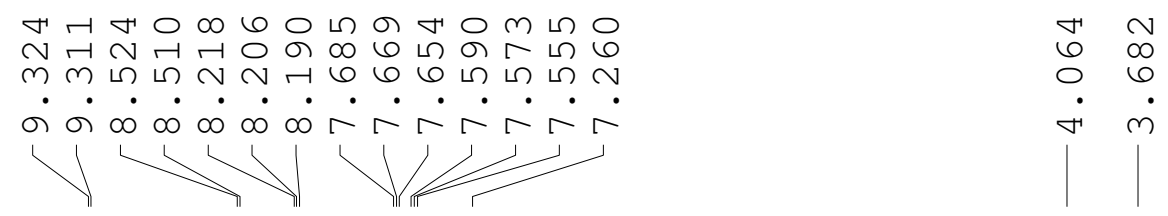

$\infty \stackrel{1}{\infty}$

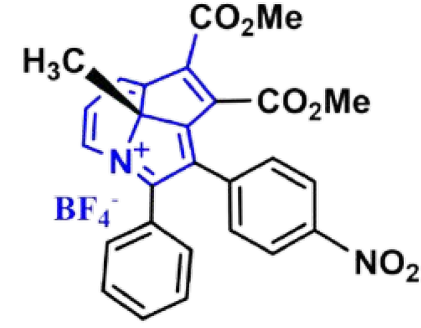

$4 \mathrm{e}$

${ }^{1} \mathrm{H} \mathrm{NMR}\left(\mathrm{CDCl}_{3}, 500 \mathrm{MHz}\right)$

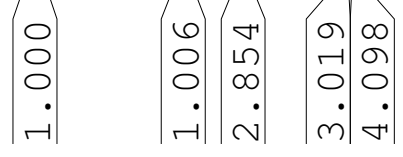

Figure S29: $1 \mathrm{H}$ NMR (500 MHz) spectrum of $4 \mathrm{e}$; *signals at $3.47 \mathrm{ppm}$ and $1.19 \mathrm{ppm}$ from residual HBF4.Et2O, 1H-NMR spectrum of a commercial sample of HBF4.Et2O is given in figure S35 


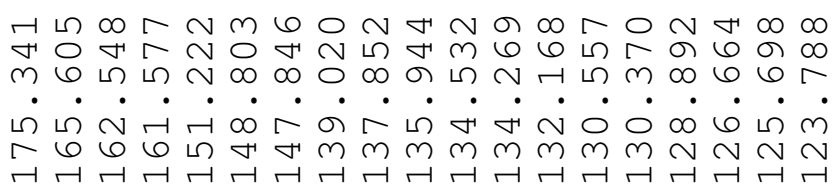

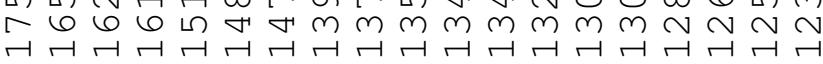

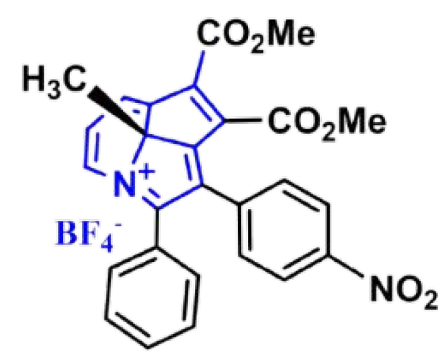

$4 \mathrm{e}$

${ }^{13} \mathrm{C} \mathrm{NMR}\left(\mathrm{CDCl}_{3}, 125 \mathrm{MHz}\right)$

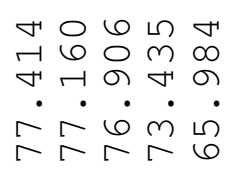

o $\curvearrowright$

$\dot{n} \dot{n} \dot{n}$

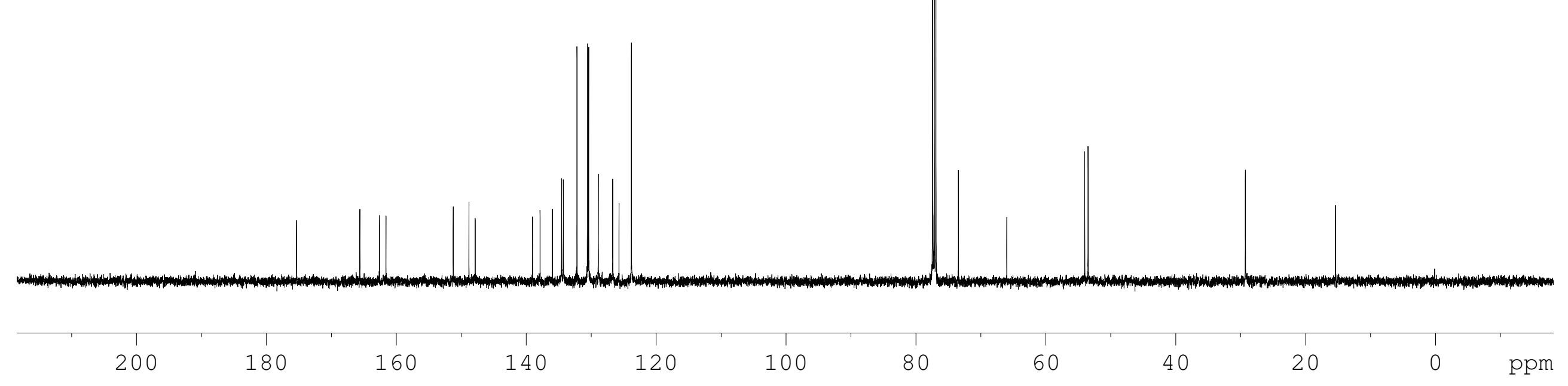
of a commercial sample of HBF4.Et2O is given in figure S36 


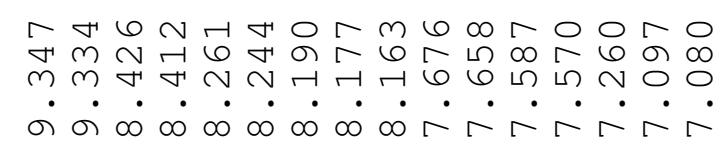

$\dot{\sigma} \dot{\sigma} \dot{\infty} \dot{\infty} \infty \dot{\infty} \dot{\infty} \dot{\infty} \dot{\sim} \dot{\sim} \dot{\sim} \dot{\sim} \dot{\sim}$

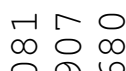

$\dot{\pi} \dot{m} \dot{m}$

$\begin{array}{lll}6 & 6 \\ 0 & 0 \\ 0 & 0 & 0\end{array}$

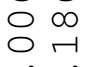

ii

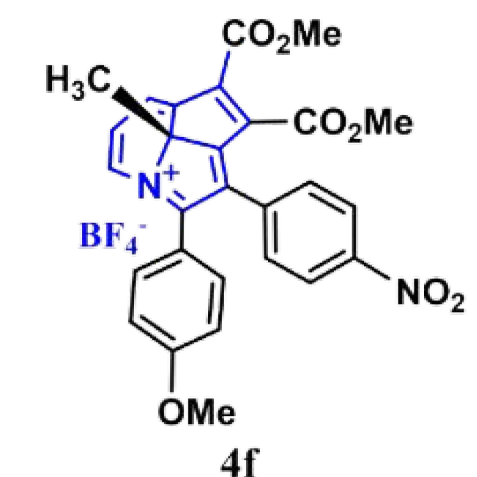

${ }^{1} \mathrm{H}$ NMR $\left(\mathrm{CDCl}_{3}, 500 \mathrm{MHz}\right)$
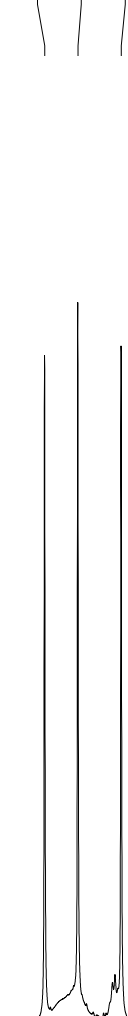

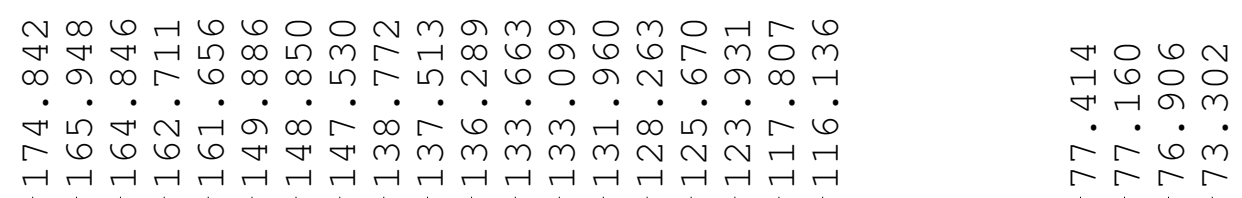

ลำ

$6 \dot{m} \dot{n}$

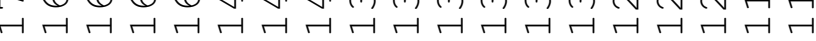

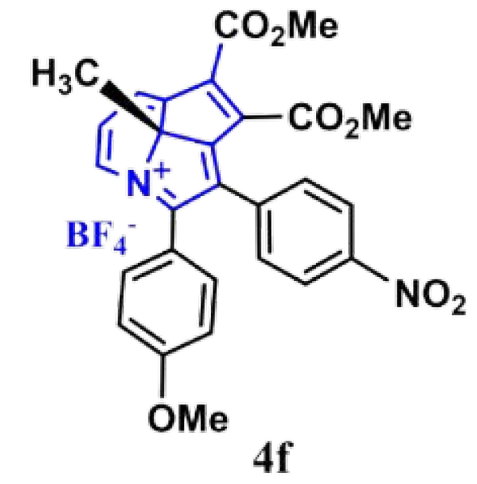

${ }^{13} \mathrm{C} \mathrm{NMR}\left(\mathrm{CDCl}_{3}, 125 \mathrm{MHz}\right)$

Whow

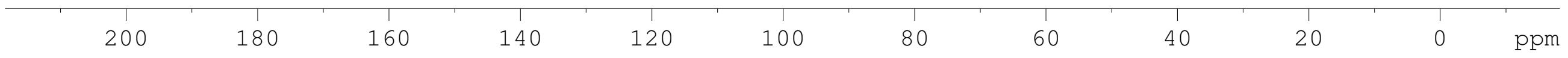

Figure S32: 13C NMR (125 MHz) spectrum of $4 \mathrm{f}$ 

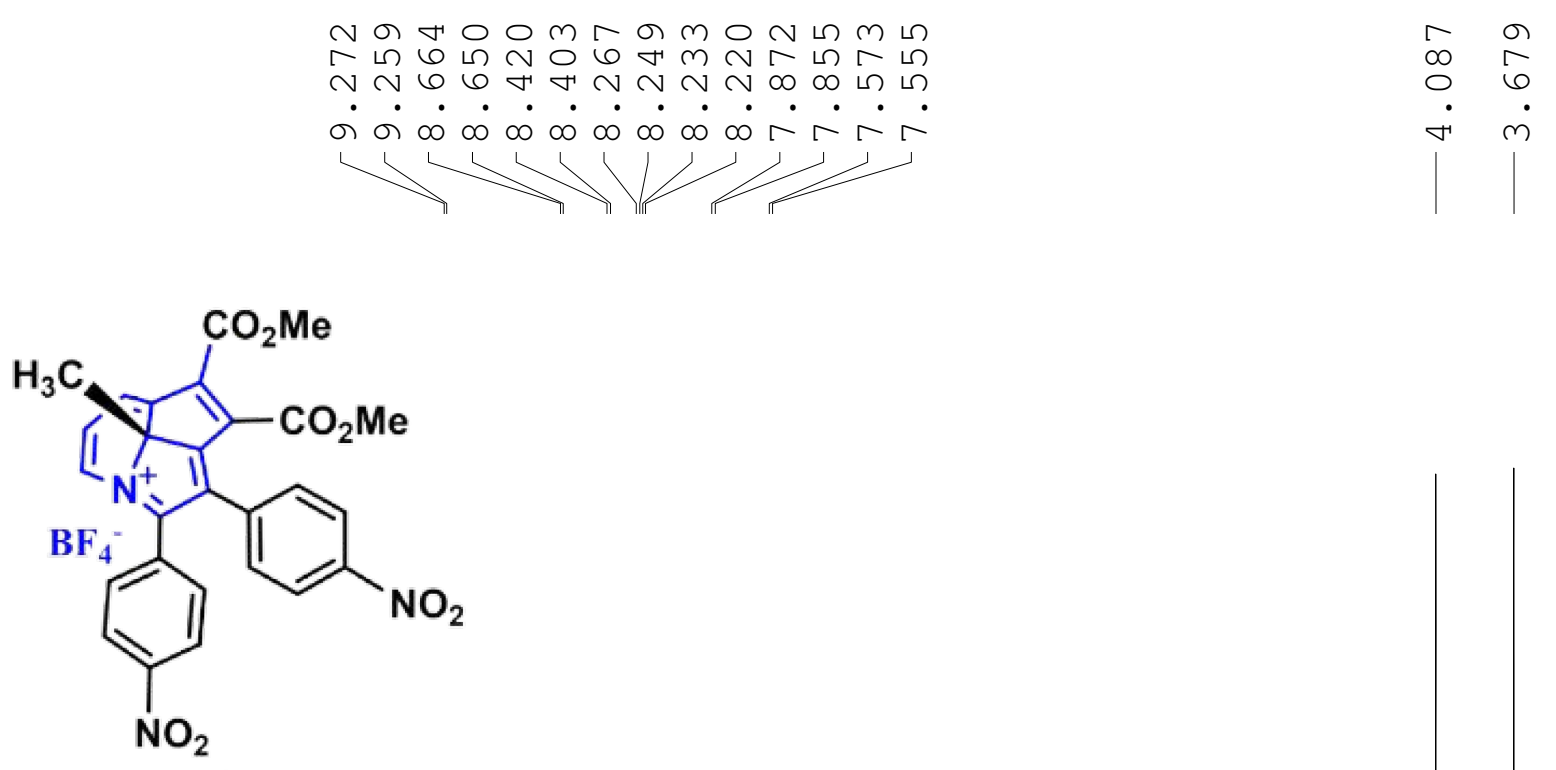

$4 \mathrm{~g}$

${ }^{1} \mathrm{H}$ NMR $\left(\mathrm{CD}_{3} \mathrm{CN}, 500 \mathrm{MHz}\right)$ 


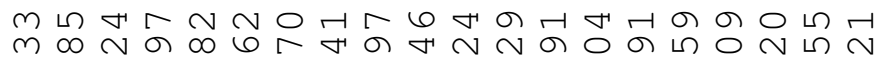
H H กmm $m$ N r

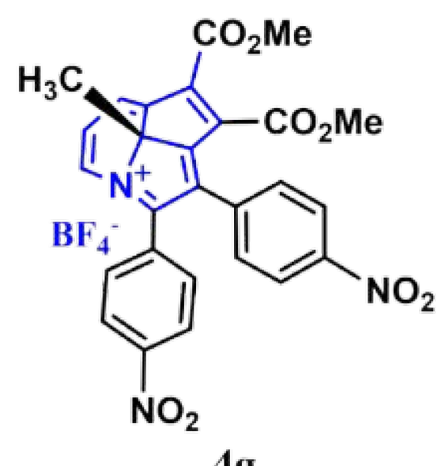

$4 \mathrm{~g}$

${ }^{13} \mathrm{C}$ NMR $\left(\mathrm{CD}_{3} \mathrm{CN}, 125 \mathrm{MHz}\right)$

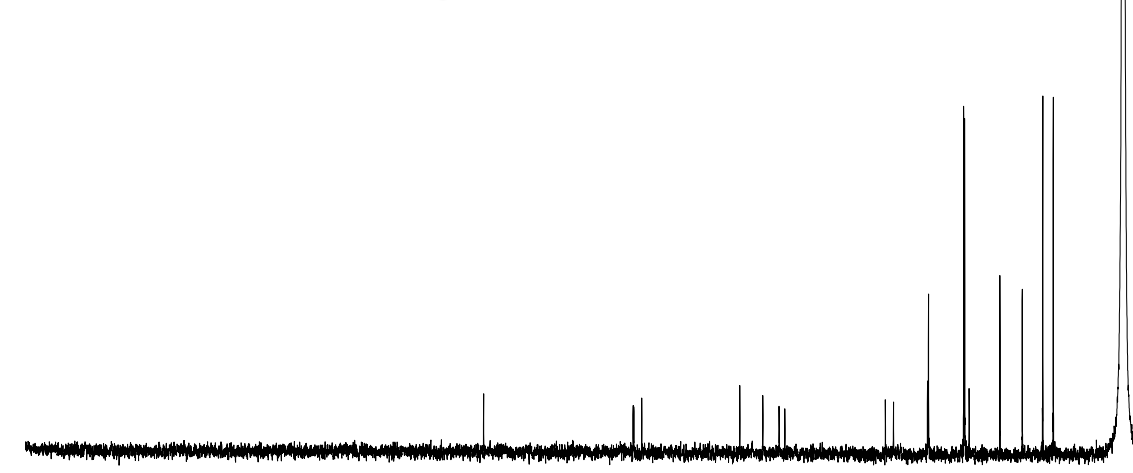




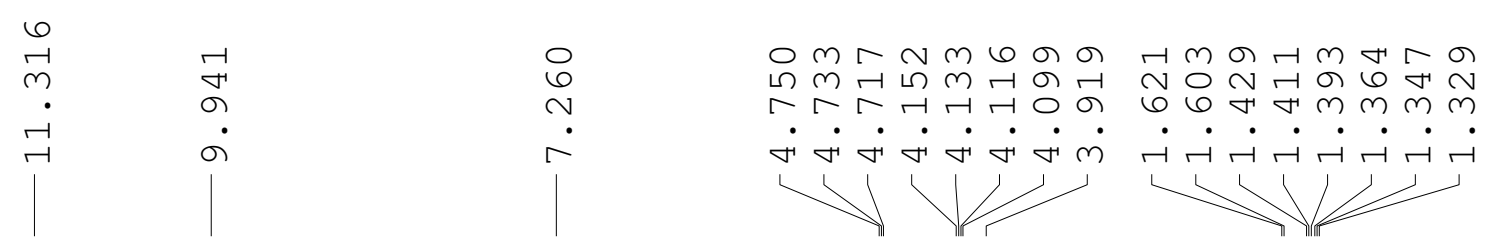

$$
\mathrm{HBF}_{4} \cdot \mathrm{Et}_{2} \mathrm{O}
$$

${ }^{\mathrm{H}} \mathrm{NMR}\left(\mathrm{CDCl}_{3}, 400 \mathrm{MHz}\right)$

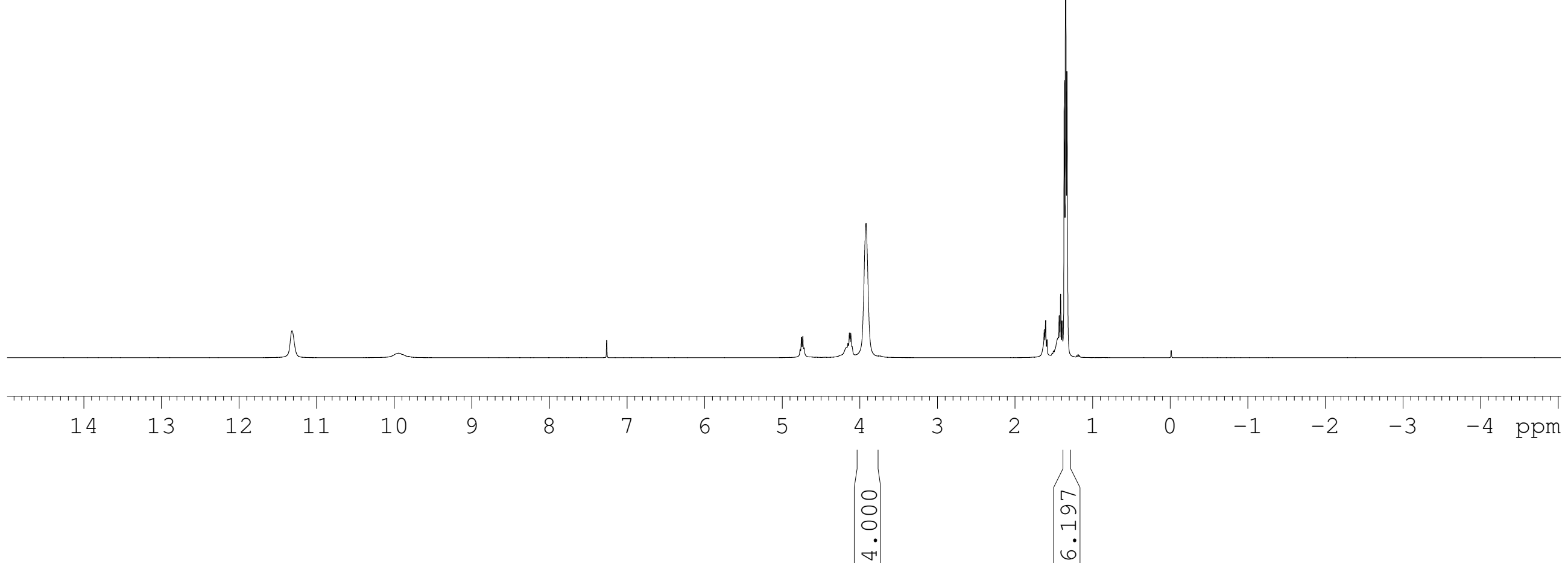

Figure S35: $1 \mathrm{H}$ NMR $(400 \mathrm{MHz})$ spectrum of commercial sample of HBF4.Et2O; signals at 1.3 and $3.9 \mathrm{ppm}$ correspond to complexed Et2O 


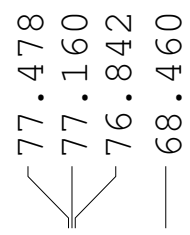

$$
\begin{aligned}
& \stackrel{\infty}{\infty} \\
& \text { कन } \\
& \dot{m}
\end{aligned}
$$

$\mathrm{HBF}_{4} \cdot \mathrm{Et}_{2} \mathrm{O}$

${ }^{13} \mathrm{C} \mathrm{NMR}\left(\mathrm{CDCl}_{3}, 100 \mathrm{MHz}\right)$

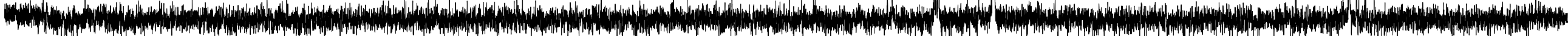

$\begin{array}{lllll}180 & 160 & 140 & 120 & 100\end{array}$

60

40

20

ppm

Figure S36: 13C NMR (100 MHz) spectrum of commercial sample of HBF4.Et2O; signals at 13.9 and 68.5 ppm correspond to complexed Et2O 
Table S8. Comparison of NMR spectra of 3a-d with 4a-d

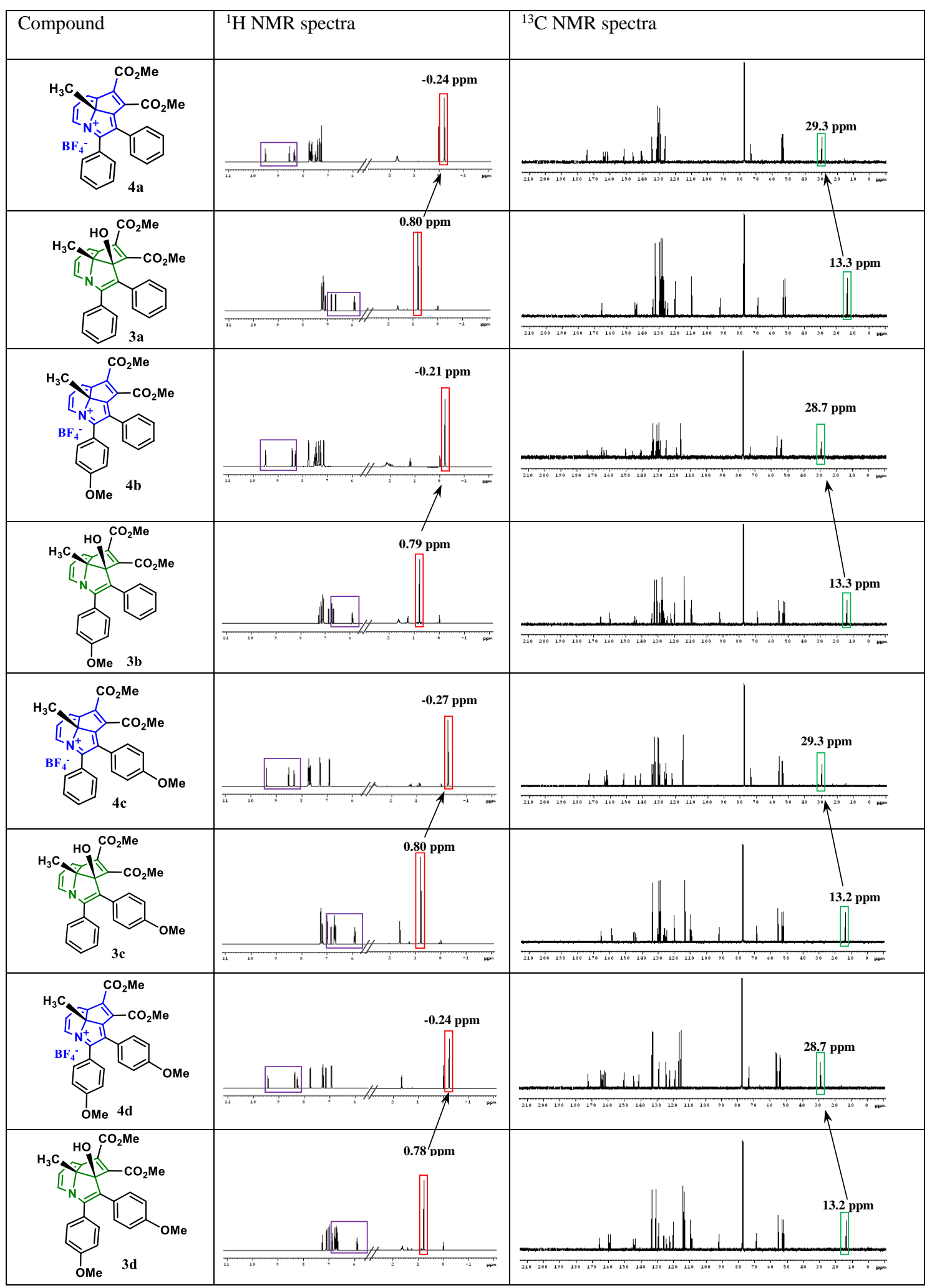




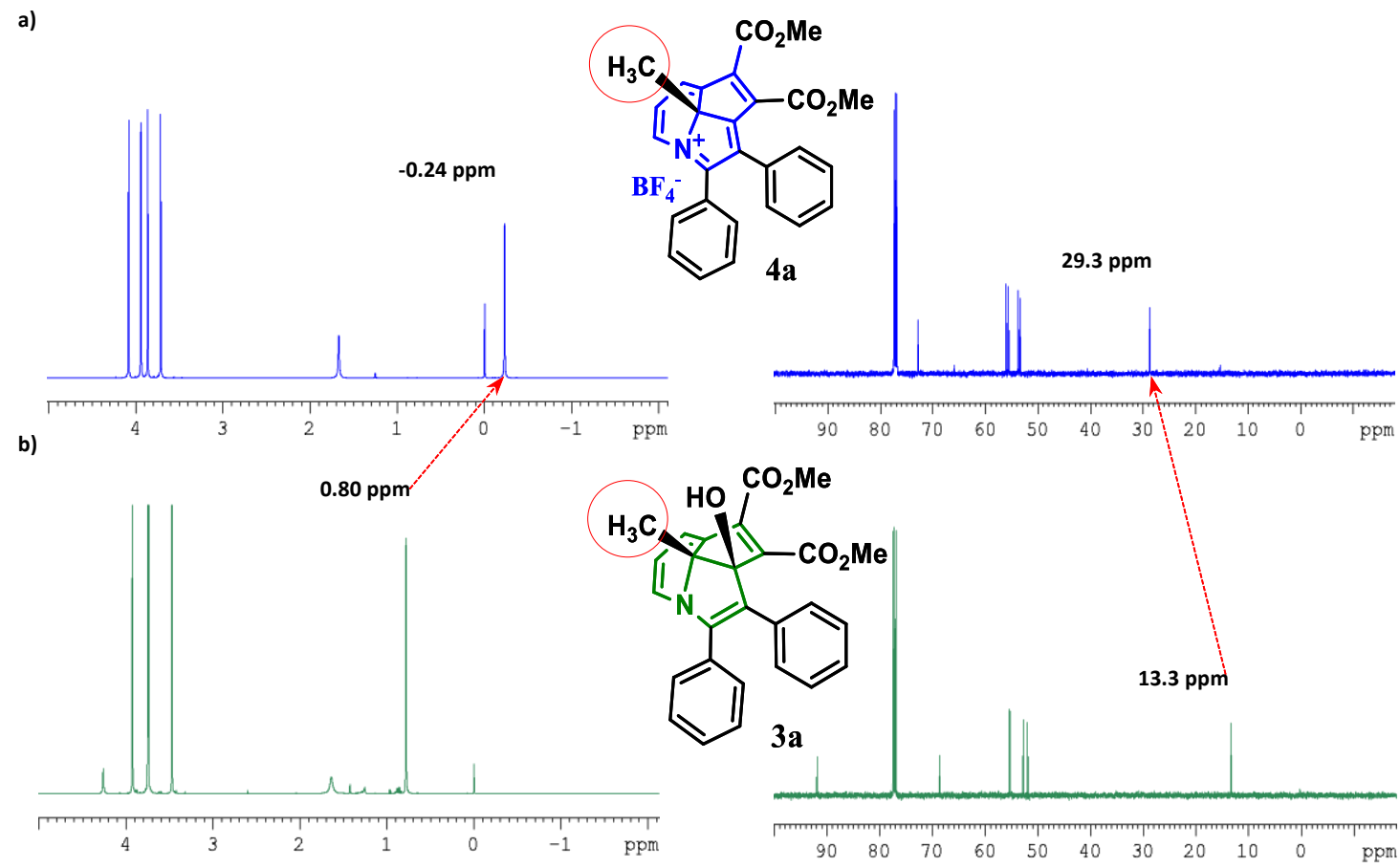

Figure S37. a) Selected region from the ${ }^{1} \mathrm{H} \&{ }^{13} \mathrm{C}$ NMR spectra of $4 \mathbf{a}\left(\mathrm{CDCl}_{3}, 500 \mathrm{MHz}\right)$ showing the change in chemical shift position of central $\mathrm{CH}_{3}$ group; b) the corresponding regions from the spectra of $\mathbf{3 a}$. 
HRMS spectra of compounds $\mathbf{4 a - g}$

Cpd. 1: $\mathrm{C27}$ H22 N 04

Compound Spectra (overlaid)

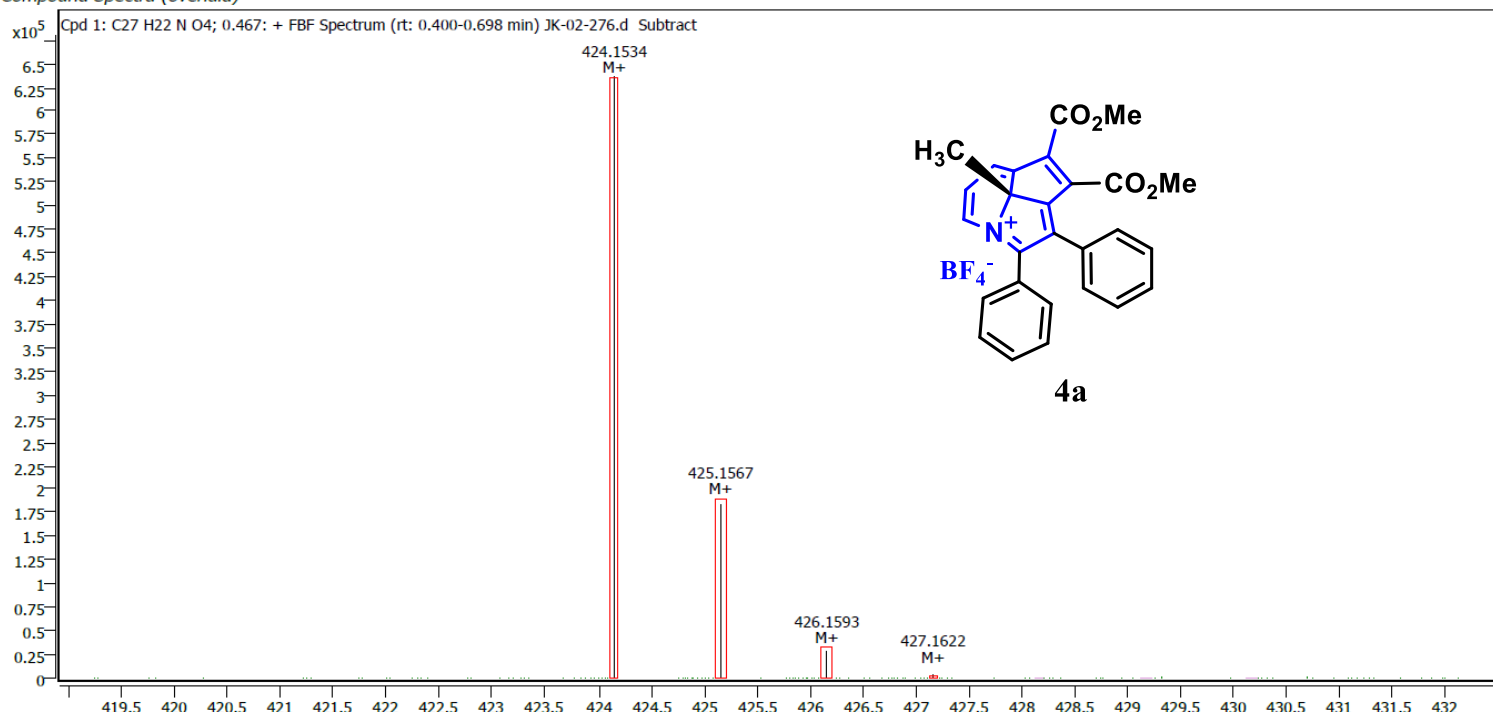

Counts vs. Mass-to-Charge $(\mathrm{m} / \mathrm{z})$

Compound ID Table

\begin{tabular}{llllllll}
\hline Cpd & Formula & Mass (Tgt) & Calc. Mass & Mass & Species & Diff(Tgt.ppm) & mDa \\
\hline 1 & C27 H22 N 04 & 424.1549 & 424.1540 & 424.1534 & M+ & -2.18 & -0.93 \\
\hline
\end{tabular}

Figure S38: HRMS spectrum of $\mathbf{4 a}$

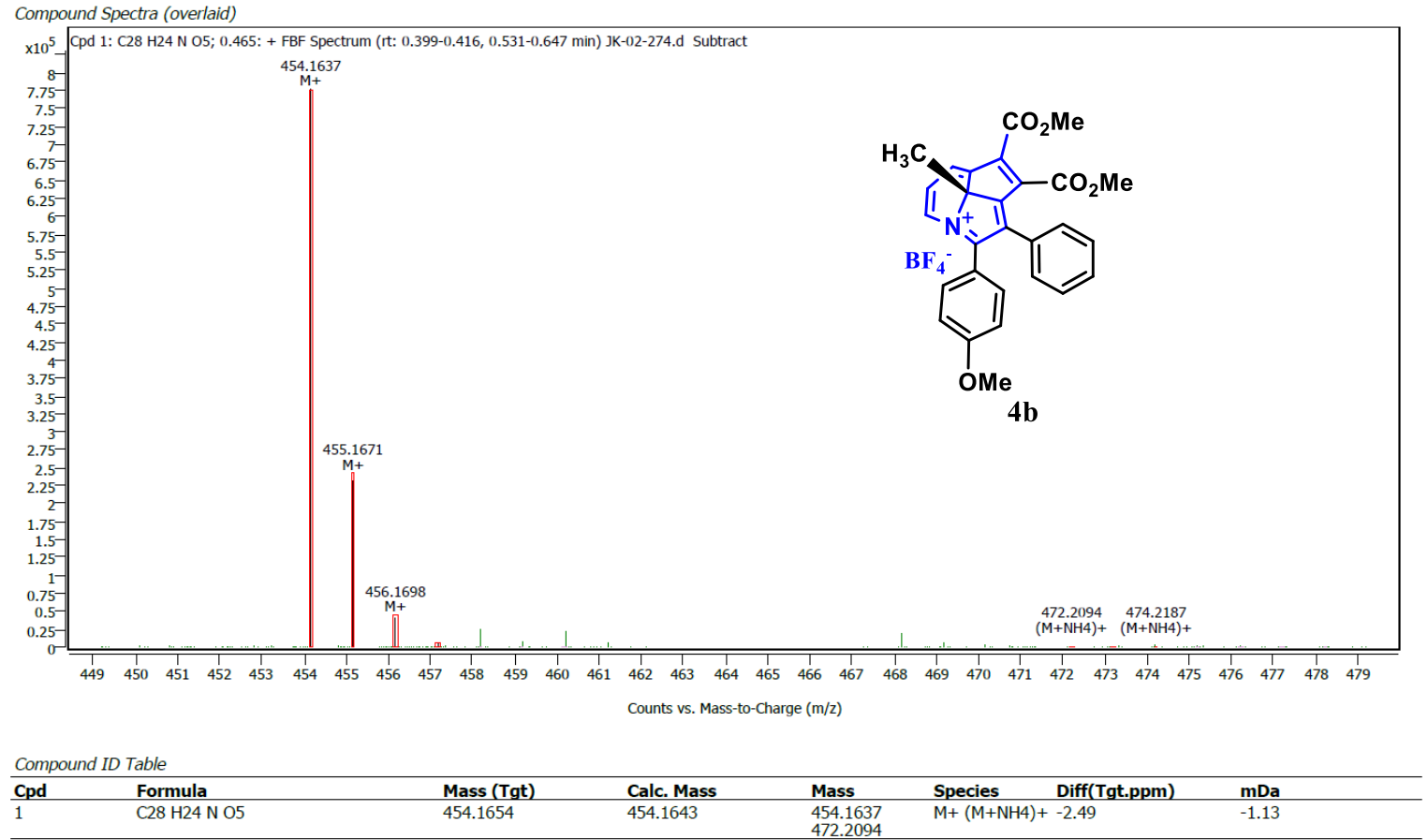

Figure S39: HRMS spectrum of $\mathbf{4 b}$ 
Compound Spectra (overlaid)

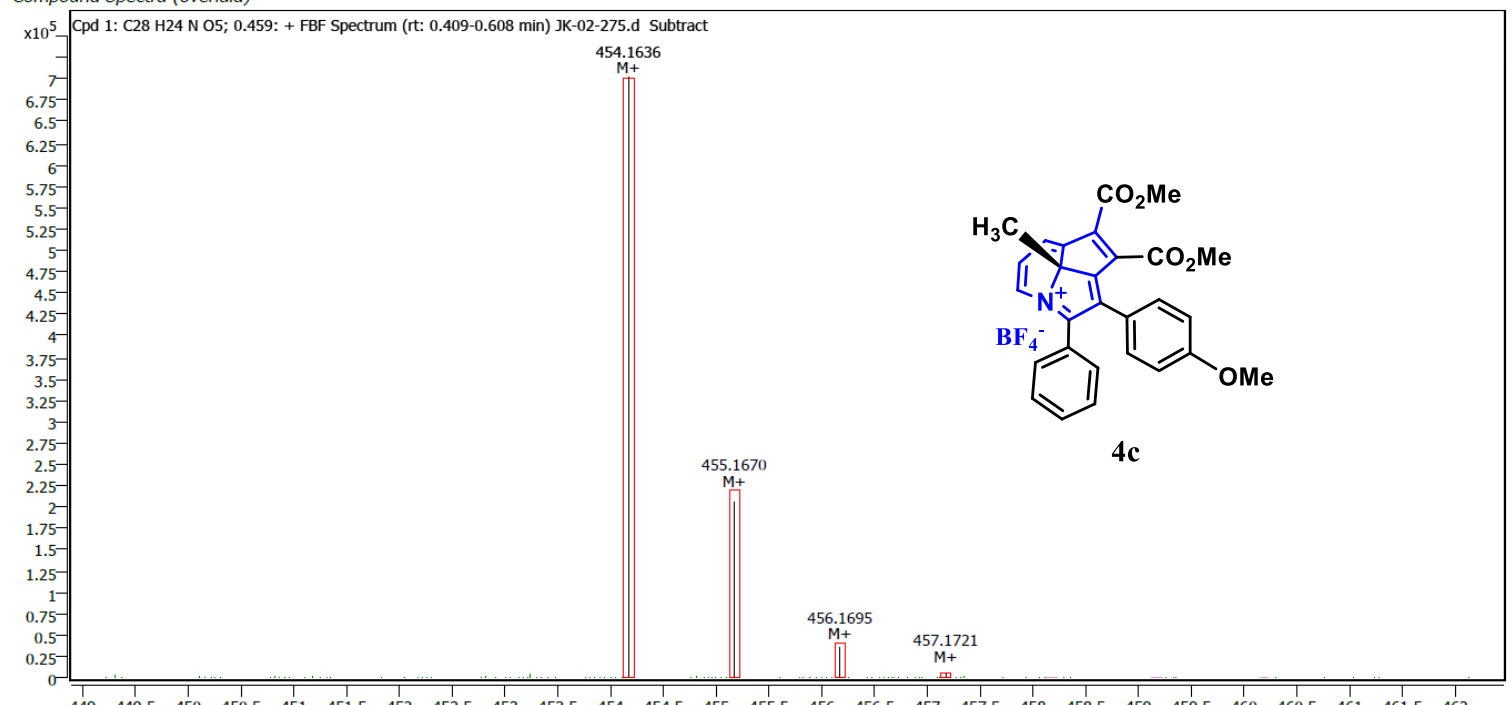

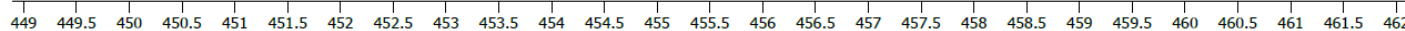
Counts vs. Mass-to-Charge $(\mathrm{m} / \mathrm{z})$

Compound ID Table

\begin{tabular}{ll}
\hline Cpd & Formula \\
\hline 1 & $\mathrm{C} 28 \mathrm{H} 24 \mathrm{~N} \mathrm{O}$
\end{tabular}

Mass (Tgt)

Calc. Mass

Mass

Species

Diff(Tgt.ppm)

$\mathrm{mDa}$

Figure S40: HRMS spectrum of $4 c$

Compound Spectra (overlaid)

$\times 10^{6}$ Cpd 1: C29 H26 N 06; 0.354: + FBF Spectrum (it: 0.239-0.255, 0.784-1.065 min) JK-280.d Subtract

$\times 10^{6}-$ cpd 1. C29 H26 N 06; 0.354: +

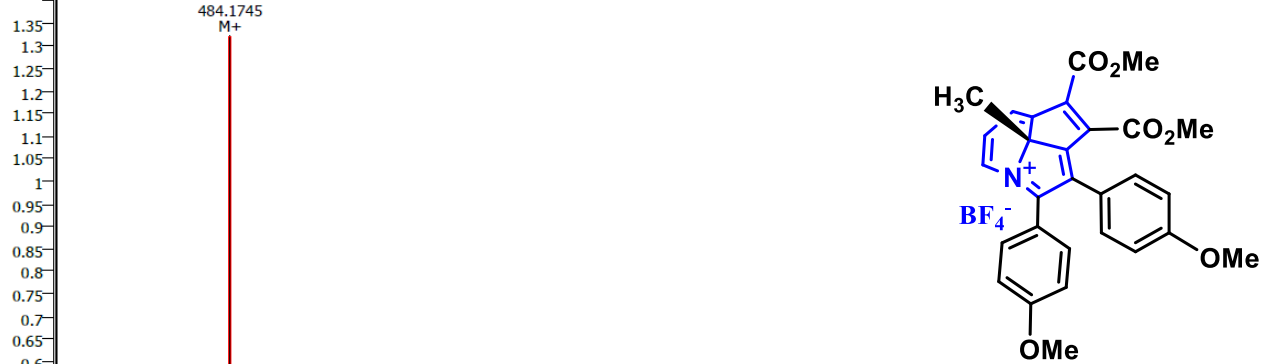

4d

507.1563
$(\mathrm{M}+\mathrm{Na})+$

523.1300
$(\mathrm{M}+\mathrm{K})+$

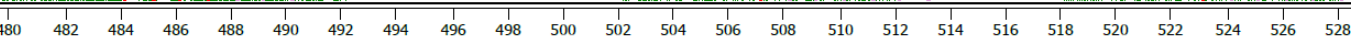

Counts vs. Mass-to-Charge $(\mathrm{m} / \mathrm{z})$

Compound ID Table

\begin{tabular}{|c|c|c|c|c|c|c|c|}
\hline Cpd & Formula & Mass (Tgt) & Calc. Mass & Mass & Species & Diff(Tgt.ppm) & $\mathrm{mDa}$ \\
\hline
\end{tabular}

Figure S41: HRMS spectrum of $\mathbf{4 d}$ 


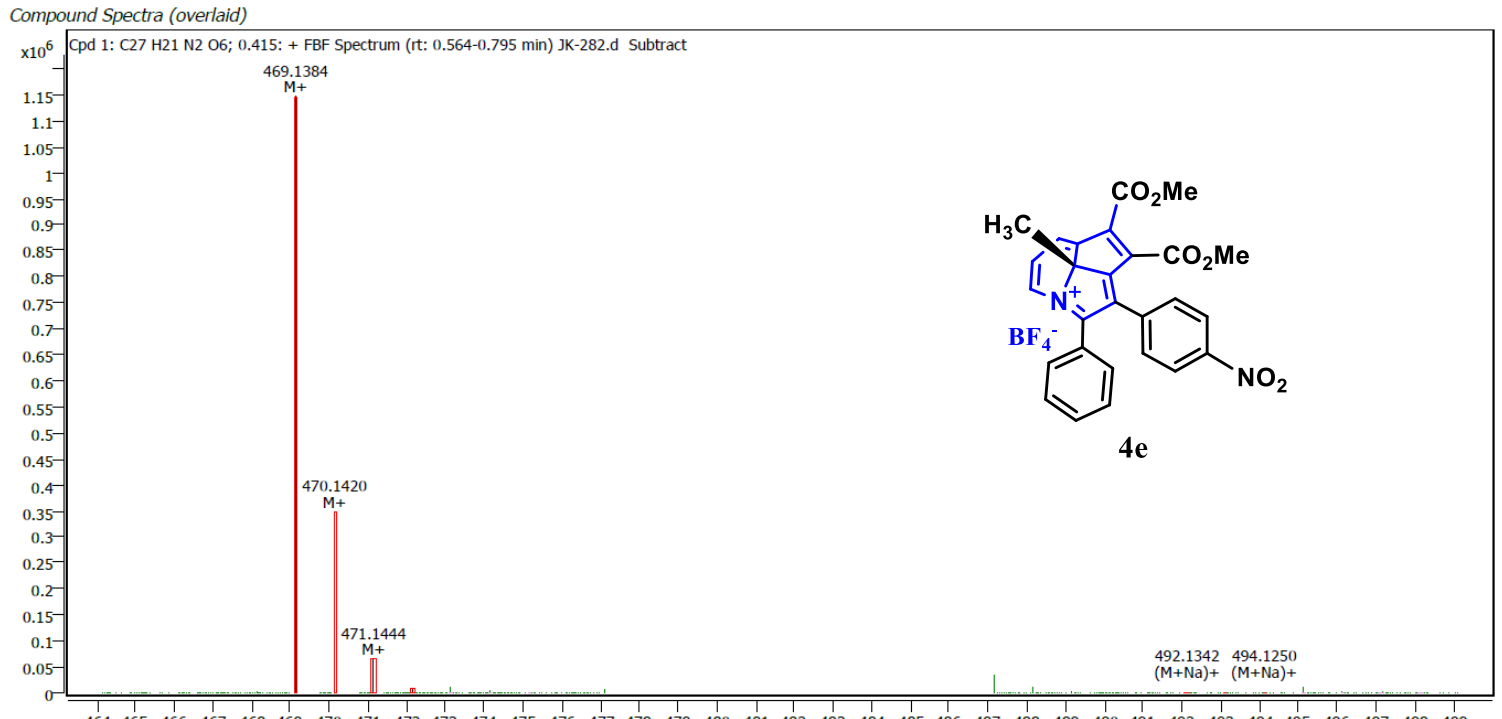

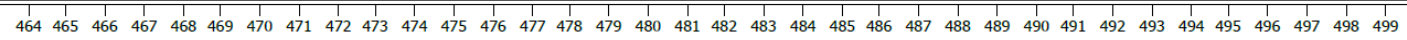
Counts vs. Mass-to-Charge $(\mathrm{m} / \mathrm{z})$

\begin{tabular}{|c|c|c|c|c|c|c|c|}
\hline 1 & $\mathrm{C} 27 \mathrm{H} 21 \mathrm{~N} 2 \mathrm{O} 6$ & 469.1400 & 469.1390 & $\begin{array}{l}469.1384 \\
492.1342\end{array}$ & $\mathrm{M}+(\mathrm{M}+\mathrm{Na})+$ & -2.01 & -0.94 \\
\hline
\end{tabular}

Figure S42: HRMS spectrum of $4 \mathbf{e}$

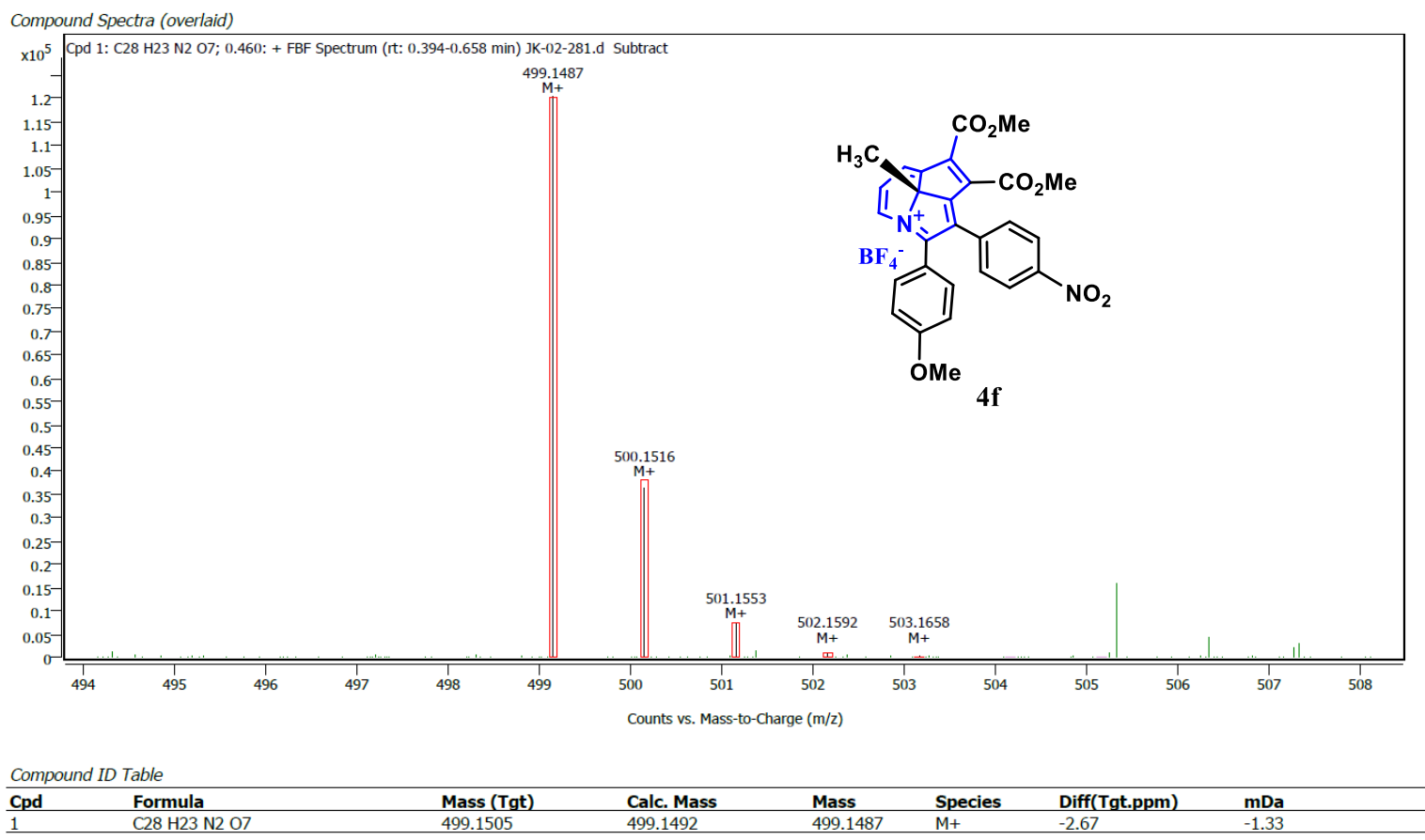

Figure S43: HRMS spectrum of $\mathbf{4 f}$ 


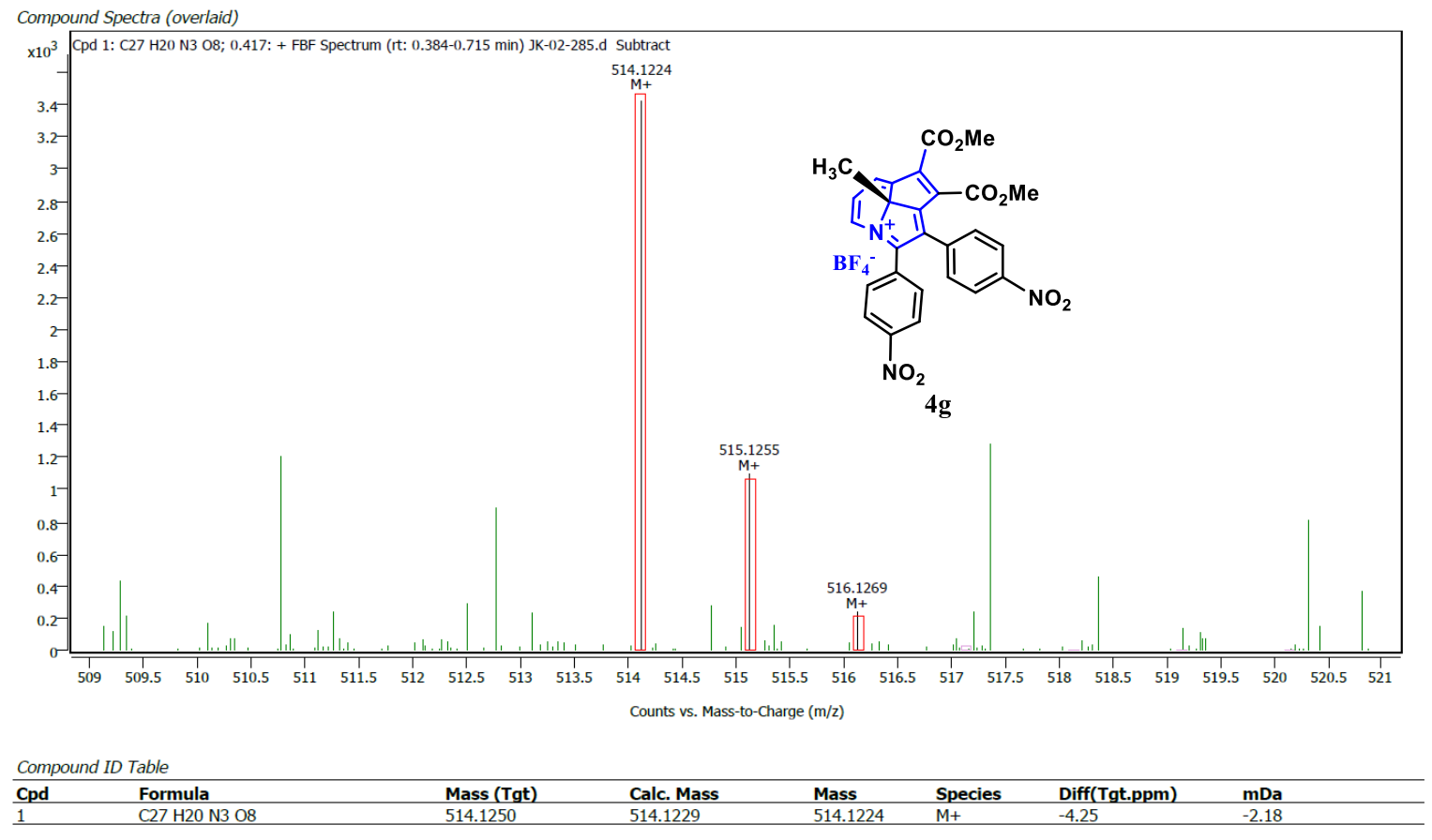

Figure S44: HRMS spectrum of $\mathbf{4 g}$

\section{References}

1. H. Cho, I. Kim, Tetrahedron, 2012, 68, 5464-5480. 